\title{
A regularity theory for intrinsic minimising fractional harmonic maps
}

\author{
James Roberts ${ }^{1}$
}

Received: 20 November 2017 / Accepted: 12 May 2018 / Published online: 25 June 2018

(C) The Author(s) 2018

\begin{abstract}
We define and develop an interior partial regularity theory for intrinsic energy minimising fractional harmonic maps from Euclidean space into smooth compact Riemannian manifolds for fractional powers strictly between zero and one. Intrinsic fractional harmonic maps are critical points of an energy whose first variation is a Dirichlet to Neumann map for the harmonic map problem on a half-space with a Riemannian metric which can degenerate/become singular along the boundary, depending on the fractional power. Similarly to the approach used to prove regularity for stationary intrinsic semi-harmonic maps, we take advantage of the connection between fractional harmonic maps and free boundary problems for harmonic maps in order to develop a partial regularity theory for the fractional harmonic maps we consider. In particular, we prove partial regularity for locally minimising harmonic maps with (partially) free boundary data on half-spaces with the aforementioned metrics up to the boundary; fractional harmonic maps then inherit this regularity. As a by-product of our methods we shed some new light on the monotonicity of the average energy of solutions of the degenerate linear elliptic equation related to fractional harmonic functions.
\end{abstract}

Mathematics Subject Classification 35J85 - 58E20 - 35S99 - 31A05 - 35B05 - 35D10 . $35 \mathrm{~J} 70 \cdot 35 \mathrm{R} 35$

Communicated by M. Struwe.

James Roberts was supported by an Engineering and Physical Sciences Research Council DTA Studentship for much of this research.

James Roberts

j.e.roberts@bath.ac.uk

1 Department of Mathematical Sciences, University of Bath, Claverton Down, Bath BA2 7AY, UK 


\section{Introduction}

Harmonic maps are critical points of the Dirichlet energy for mappings of Riemannian manifolds and are non-linear analogues of solutions to Laplace's equation. They play an important role in geometry and one of the most fundamental aspects of the theory of harmonic maps, which is intimately connected to the geometry and topology of the codomain manifold $N$, is their regularity. For maps with domains of dimension 2, the Dirichlet energy is invariant under conformal transformations of the domain; Hélein proved that harmonic maps are smooth in this case [21]. The fact that harmonic maps are smooth on domains of dimension two is also now a consequence of a result of Rivière [34] asserting the continuity of critical points of functionals with conformally invariant Lagrangian on two dimensional domains. Motivated by Rivière's regularity result, at least partially, Da Lio and Rivière [8] introduced the notion of fractional harmonic mappings of manifolds; on a domain of dimension one these maps are critical points of functionals which satisfy a type of conformal invariance. Such maps generalise the notion of both harmonic mappings of manifolds and fractional harmonic functions (maps $u$ with $(-\Delta)^{s} u=0$ ) and Da Lio and Rivière showed the maps they considered are smooth on domains of one dimension.

Since the results of Da Lio and Rivière, fractional harmonic mappings of manifolds have been increasingly studied in recent years, particularly pertaining to their regularity. The purpose of this article is to extend the notion of a type of fractional harmonic map introduced by Moser [30]. In particular, we generalise the notion of intrinsic $\frac{1}{2}$-harmonic maps to all powers in $(0,1)$ and consider the regularity of a class of such maps. In contrast to other types of fractional harmonic maps, the maps we consider are intrinsic meaning that they are derived from an energy which only depends on the geometry of the target $N$ and not on the embedding of $N$ into Euclidean space. As we will discuss in more detail subsequently, we take advantage of the connection between free boundary harmonic maps and fractional harmonic maps to establish regularity for the latter. The results we obtain and methods we use lay the foundation for future studies into the connection between the regularity and singularities of intrinsic fractional harmonic maps, free boundary harmonic maps and the geometric and topological properties of $N$.

In order to motivate our definition of fractional harmonic mappings of manifolds, we first outline the pertinent aspects of the theory of fractional harmonic functions from Euclidean space into $\mathbb{R}$. Let $m \in \mathbb{N}$ and $\mathbb{R}_{+}^{m+1}=\mathbb{R}^{m} \times(0, \infty)$. Caffarelli and Silvestre [2] established that, for given boundary data $u: \partial \mathbb{R}_{+}^{m+1} \rightarrow \mathbb{R}$, solutions $v: \mathbb{R}_{+}^{m+1} \rightarrow \mathbb{R}$ of the Dirichlet problem:

$$
\operatorname{div}\left(x_{m+1}^{\beta} \nabla v\right)=0 \text { in } \mathbb{R}_{+}^{m+1} \text { and }\left.v\right|_{\partial \mathbb{R}_{+}^{m+1}}=u
$$

satisfy $(-\Delta)^{\frac{1-\beta}{2}} u=\partial_{m+1}^{\beta} v:=-\left.\left(x_{m+1}^{\beta} \partial_{m+1} v\right)\right|_{\partial \mathbb{R}_{+}^{m+1}}$, where $(-\Delta)^{s}$ is the fractional Laplace operator of order $s \in(0,1)$. One way to prove this result is to establish that

$$
C\left\|(-\Delta)^{\frac{s}{2}} u\right\|_{L^{2}\left(\partial \mathbb{R}_{+}^{m+1}\right)}^{2}=\inf \left\{E^{\beta}(v):\left.v\right|_{\partial \mathbb{R}_{+}^{m+1}}=u\right\},
$$

where $E^{\beta}(v):=\frac{1}{2} \int_{\mathbb{R}_{+}^{m+1}} x_{m+1}^{\beta}|\nabla v|^{2} \mathrm{~d} x, s=\frac{1-\beta}{2}, C=C(m, s)$ and the infimum is taken over a suitable Sobolev space. We conclude that the first variation of the preceding functionals, respectively $u \mapsto(-\Delta)^{\frac{1-\beta}{2}} u$ and $u \mapsto \partial_{m+1}^{\beta} v$, must therefore coincide. Furthermore, if we wish to study $u$ with $(-\Delta)^{s} u=0$, then we may equivalently consider minimisers (or even critical points) of $E^{\beta}$ which satisfy $\left.v\right|_{\partial \mathbb{R}_{+}^{m+1}}=u$ and $\partial_{m+1}^{\beta} v=0$. It further follows from 
(1.2) that fractional harmonic functions $u: \mathcal{O} \rightarrow \mathbb{R}$, where $\mathcal{O} \subset \partial \mathbb{R}_{+}^{m+1}$ is a domain, may reasonably be defined as critical points of either $\left\|(-\Delta)^{\frac{s}{2}} u\right\|_{L^{2}(\mathcal{O})}^{2}$ or $\inf \left\{E^{\beta}(v):\left.v\right|_{\mathcal{O}}=u\right\}$ (which are no longer equivalent). In order to define intrinsic fractional harmonic maps, we will work with a modification of the latter energy. For $u: \mathcal{O} \rightarrow \mathbb{R}$, the first variation of $\inf \left\{E^{\beta}(v):\left.v\right|_{\mathcal{O}}=u\right\}$ is the Dirichlet to Neumann map $u \mapsto-\left.\left(x_{m+1}^{\beta} \partial_{m+1} v\right)\right|_{\mathcal{O}}$ but we may no longer identify this with a fractional Laplace operator. Moreover, $v$ satisfies (1.1) but with $\left.v\right|_{\mathcal{O}}=u$.

We generalise the notion of fractional harmonic function to mappings $u: \mathcal{O} \rightarrow N$ in the following way, where $\mathcal{O} \subset \partial \mathbb{R}_{+}^{m+1}$ is an open domain but not necessarily all of $\mathbb{R}_{+}^{m+1}$ and $N$ is a smooth compact Riemannian manifold. For technical reasons, we assume henceforth that $N$ is isometrically embedded in $\mathbb{R}^{n}$ for some $n$, which can always be achieved for smooth compact $N$ by the theorem of Nash [31]. The forthcoming definition will be made rigorous at the beginning of Sect. 3. Define

$$
I^{\beta}(u)=\inf \left\{E^{\beta}(v):\left.v\right|_{\mathcal{O}}=u, v \in \dot{W}_{\beta}^{1,2}\left(\mathbb{R}_{+}^{m+1} ; N\right)\right\}
$$

where $\beta \in(-1,1), \dot{W}_{\beta}^{1,2}\left(\mathbb{R}_{+}^{m+1} ; N\right):=\left\{v \in \dot{W}_{\beta}^{1,2}\left(\mathbb{R}_{+}^{m+1} ; \mathbb{R}^{n}\right): v(x) \in N\right.$ for a.e $\left.x \in \mathbb{R}_{+}^{m+1}\right\}$ and $\dot{W}_{\beta}^{1,2}\left(\mathbb{R}_{+}^{m+1} ; \mathbb{R}^{n}\right)$ is the homogeneous Sobolev space with the square root of $E^{\beta}$ as the norm. If we set $\mathcal{O}=\partial \mathbb{R}_{+}^{m+1}$ and $N$ is replaced by $\mathbb{R}$ then $I^{\beta}$ reduces to the energies considered by Caffarelli and Silvestre. Moreover, $I^{\beta}$ is independent of the choice of embedding of $N$ into Euclidean space since $E^{\beta}$ is invariant under isometries of $N$. Hence we define critical points of $I^{\beta}$ as intrinsic $\frac{1-\beta}{2}$-harmonic maps.

Our main result regarding fractional harmonic maps, see Theorem 3.8 in Sect. 3, asserts:

Theorem 1.1 Fix $\mathcal{O} \subset \partial \mathbb{R}_{+}^{m+1}$. When $m \geq 3$ let $\beta \in(-1,1)$ and when $m=2$ let $\beta \in$ $\left(-3^{-1}, 1\right)$. Suppose $u: \mathcal{O} \rightarrow N$ is a $\frac{1-\beta}{2}$-harmonic map that locally minimises $I^{\beta}$ in $\mathcal{O}$. Then $u$ is smooth with the possible exception of a set of points with vanishing $m+\beta-1$-dimensional Hausdorff measure.

We observe that the domain of $I^{\beta}$ plays a role in the regularity theory. We will take the domain of $I^{\beta}$, again see Sect. 3 for details, to be the image of $\dot{W}_{\beta}^{1,2}\left(\mathbb{R}_{+}^{m+1} ; N\right)$ under some trace operator. The domain we choose does not necessarily coincide with the fractional Sobolev space $H^{\frac{1-\beta}{2}}(\mathcal{O} ; N)$; there are circumstances in which it is possible to construct $H^{\frac{1-\beta}{2}}(\mathcal{O} ; N)$ maps which do not have extensions to $\mathbb{R}_{+}^{m+1}$. This can happen for topological reasons, for example, such as when $N$ is not simply connected [1]. We further note that the bound on the Hausdorff dimension of the singular set stated in the theorem may not be optimal; the given dimension is that which one would conjecture for $u$ which are critical points of $I^{\beta}$ with respect to inner and outer variations and is consistent with the scale-invariance of the problem. We expect that the dimension bound on the singular set can be improved using our constructions in Sect. 4 and similar arguments to Chapter 3 of [39] for example. It is also possible that our choice to minimise over $v \in \dot{W}_{\beta}^{1,2}\left(\mathbb{R}_{+}^{m+1} ; N\right)$ may preclude some singularities, for example the map $v=\left|\left(x^{\prime}, x_{m+1}\right)\right|^{-1}\left(x^{\prime}, x_{m+1}\right)$ is not an admissible extension of $u=\left|x^{\prime}\right|^{-1} x^{\prime}$ as it does not have finite $E^{\beta}$ energy. However, the arguments we use are local and we expect our methods to apply to modifications of $I^{\beta}$ which allow extensions $v$ with only locally finite $E^{\beta}$ energy. The restriction $\beta>-3^{-1}$ when $m=2$ is of a technical nature and results from the use of a Sobolev embedding theorem to identify elements of $\dot{W}_{\beta}^{1,2}\left(\mathbb{R}_{+}^{m+1} ; \mathbb{R}^{n}\right)$ with functions, so that we may require they take values in $N$ almost everywhere. It is possible that this restriction can be lifted with the use of an appropriate embedding theorem, we are not aware 
of such a result. An alternative would be to replace $\dot{W}_{\beta}^{1,2}$ with a Sobolev space of measurable functions from the outset (as opposed to representatives of Cauchy sequences). We further note that we do not consider the case $m=1$ and will indicate our reasons subsequently.

To prove Theorem 1.1 we will take advantage of the connection between the extensions $v: \mathbb{R}_{+}^{m+1} \rightarrow N$ of fractional harmonic $u: \mathcal{O} \rightarrow N$ and free boundary harmonic maps from $\mathbb{R}_{+}^{m+1}$ to $N$, similarly to Moser [30]. Moser observed that whenever $I^{0}$ is differentiable at $u$, its first variation is the Dirichlet to Neumann map $\Lambda:\left.u \mapsto\left(-\partial_{m+1} v\right)\right|_{\mathcal{O}}$ where $v: \mathbb{R}_{+}^{m+1} \rightarrow N$ is a harmonic map with $I^{0}(u)=E^{0}(v)$. Hence, intrinsic $\frac{1}{2}$-harmonic maps satisfy $\Lambda u=$ $\left.\left(-\partial_{m+1} v\right)\right|_{\mathcal{O}}=0$, which may be interpreted as a zero Neumann condition on $\mathcal{O}$ for any $v$ which minimises $E^{0}$ among maps with $u$ as boundary values on $\mathcal{O}$. Using this observation Moser established (partial) regularity for stationary $\frac{1}{2}$-harmonic $u$ : $\mathcal{O} \rightarrow N$, which are critical points of $I^{0}$ with respect to inner and outer variations, using regularity theory for stationary free boundary harmonic maps $v: \mathbb{R}_{+}^{m+1} \rightarrow N$ up to the free boundary $\mathcal{O}$. He showed the maps he considered are smooth when $m=1$ and smooth with the possible exception of a set of vanishing Hausdorff dimension $m-1$ when $m \geq 2$. The following theorem is an abridged version of Theorem 4.3 in Sect. 4 which is our main theorem regarding the extensions $v$ of fractional harmonic $u$.

Theorem 1.2 Suppose $u: \mathcal{O} \rightarrow N$ locally minimises $I^{\beta}$ and suppose $v$ satisfies $I^{\beta}(u)=$ $E^{\beta}(v)$. There exists sets $\Sigma_{\text {int }} \subset \mathbb{R}_{+}^{m+1}$ and $\Sigma_{\text {bdry }} \subset \mathcal{O}$ such that the following holds. The set $\Sigma_{\text {int }}$ is relatively closed in $\mathbb{R}_{+}^{m+1}$ and has Hausdorff dimension at most $m-2$. The set $\Sigma_{\text {bdry }}$ is relatively closed in $\mathcal{O}$ and has vanishing $m+\beta-1$-dimensional Hausdorff measure. The set $\Sigma:=\Sigma_{\text {int }} \cup \Sigma_{\text {bdry }}$ is relatively closed in $\mathbb{R}_{+}^{m+1} \cup \mathcal{O}$ and has vanishing $m+\beta-1$-dimensional Hausdorff measure. Furthermore, we have $v \in C^{\infty}\left(\mathbb{R}_{+}^{m+1} \backslash \Sigma_{\text {int }} ; N\right)$, $v \in C_{\text {loc }}^{0,1}\left(\left(\mathbb{R}_{+}^{m+1} \cup \mathcal{O}\right) \backslash \Sigma ; N\right)$ and for every multi-index $\alpha^{\prime} \in \mathbb{N}^{m+1}$ with $\alpha_{m+1}^{\prime}=0$ we have $D^{\alpha^{\prime}} v \in C_{\text {loc }}^{0,1}\left(\left(\mathbb{R}_{+}^{m+1} \cup \mathcal{O}\right) \backslash \Sigma ; \mathbb{R}^{n}\right)$.

Synonymously with our discussion following Theorem 1.1, we observe that the dimension bound on the singular set of $v$ in $\mathcal{O}$ may not be optimal. The main purpose of this article is to give a proof of Theorem 1.2 which, to our knowledge, is new for all $\beta \neq 0$. When $\beta=0$, existing theories actually improve the bound we have given on the dimension of the singular set in $\mathcal{O}$; this improvement comes from a dimension reduction argument as mentioned previously. This translates to a lower bound on the dimension of the singular set of $u$. In particular, as a consequence of the results of Hardt and Lin [18] and Duzaar and Steffen [10,11], any $v: \mathbb{R}_{+}^{m+1} \rightarrow N$ with $I^{0}(u)=E^{0}(v)$ are smooth up to $\mathcal{O}$ away from a set in $\mathcal{O}$ of Hausdorff dimension at most $\operatorname{dim} \mathcal{O}-3$. They also give examples of minimisers with singular sets in $\mathcal{O}$ which have precisely the stated Hausdorff dimensions, thus showing that the dimension bounds for the singular set are optimal.

We would like to take advantage of Moser's observation regarding the connection between fractional harmonic $u$ and harmonic $v$ with free boundary data in order to develop a theory for intrinsic fractional harmonic maps for powers in $(0,1)$. In $\mathbb{R}_{+}^{m+1}$ we are free to utilise the theory of harmonic mappings of manifolds to obtain the stated regularity in Theorem 1.2, provided the energies $E^{\beta}$ correspond to the energy for harmonic mappings from $\left(\mathbb{R}_{+}^{m+1}, g\right)$ to $N$ for some metric $g$. To see that this is the case and facilitate our subsequent analysis, we first recall the definition of harmonic maps for a class of conformally Euclidean metrics on $\mathbb{R}_{+}^{m+1}$. Let $\left(\mathbb{R}_{+}^{m+1}, g\right)$ be a smooth Riemannian manifold and suppose $g=f\left(x_{m+1}\right) \delta$, where $\delta$ is the Euclidean metric, $f: \mathbb{R}_{+}^{m+1} \rightarrow(0, \infty)$ is smooth and $x_{m+1}$ is the $m+1$ th Euclidean coordinate. Let $W^{1,2}(\Omega ; N):=\left\{v \in W^{1,2}\left(\Omega ; \mathbb{R}^{n}\right): v(x) \in N\right.$ for almost every $\left.x \in \Omega\right\}$, 
where $\Omega \subset \mathbb{R}_{+}^{m+1}$ is open. If $x_{1}, \ldots, x_{m+1}$ are Euclidean coordinates on $\Omega$, the energy density of $v \in W^{1,2}(\Omega ; N)$ is given by $e(v)=f^{-1} \sum_{i}\left\langle\frac{\partial v}{\partial x_{i}}, \frac{\partial v}{\partial x_{i}}\right\rangle:=f^{-1}\langle\nabla v, \nabla v\rangle=$ $f^{-1}|\nabla v|^{2}$, where $\langle\cdot, \cdot\rangle$ is the Euclidean inner product in $\mathbb{R}^{n}$. The energy of $v$, see [22], is defined as

$$
E_{g}(v)=\frac{1}{2} \int_{\Omega} f^{-1}|\nabla v|^{2}(\operatorname{det}(f \delta))^{\frac{1}{2}} \mathrm{~d} x=\frac{1}{2} \int_{\Omega} f^{\frac{m-1}{2}}|\nabla v|^{2} \mathrm{~d} x .
$$

Observe that when $m=1$, for this class of metric $g$ we have $E_{g} \equiv E_{\delta}$. The Euler-Lagrange equations for $E_{g}$ have the form

$$
f^{-\frac{(m+1)}{2}} \operatorname{div}\left(f^{\frac{(m-1)}{2}} \nabla v\right)+f^{-1} A(v)(\nabla v, \nabla v)=0 \text { in } \Omega
$$

where $A$ is the second fundamental form of $N, A(v)(\nabla v, \nabla v):=\sum_{i} A(v)\left(\partial_{i} v, \partial_{i} v\right)$ and $\operatorname{div}, \nabla$ are the Euclidean divergence and gradient operators. Critical points of $E_{g}$ in $W^{1,2}(\Omega ; N)$ with respect to the dependent variable (weakly) satisfy (1.4) and are called (weakly) harmonic maps with respect to $g$.

When $m \geq 2$ the Dirichlet energy $E^{\beta}$ is the energy for maps $\mathbb{R}_{+}^{m+1} \rightarrow N$ where $\mathbb{R}_{+}^{m+1}$ is equipped with a Riemannian metric $x_{m+1}^{\alpha} \delta$; it follows from (1.3) that $\beta=\frac{\alpha(m-1)}{2}$. When $m=1$, any metric of the form $x_{m+1}^{\alpha} \delta$ gives rise to the energy $E^{0}$ in view of (1.3). It is therefore not possible that $E^{\beta}$ corresponds to the energy of mappings from $\left(\mathbb{R}_{+}^{m+1}, x_{m+1}^{\alpha} \delta\right)$ for $\beta \neq 0$ when $m=1$ and, in this case, we would need a different approach. We do not consider this further in this article and hence assume $m \geq 2$.

Near the boundary $\mathcal{O}$ we would like to apply the theory for free boundary harmonic mappings of Riemannian manifolds in order to obtain regularity for fractional harmonic maps defined on $\mathcal{O}$. However, for fractional powers $\frac{1-\beta}{2} \in(0,1)$ with $\beta \neq 0$ (i.e fractional powers in $(0,1)$ other than $\left.\frac{1}{2}\right)$ the metrics $x_{m+1}^{\alpha} \delta$ may degenerate or become singular along the boundary depending on $\beta=\frac{\alpha(m-1)}{2}$. Known theory for free boundary harmonic maps is not applicable in this case and the main focus of this article will be to develop a regularity theory in this context.

Without geometric constraints on the target manifold $N$ (such as requiring $N$ has nonpositive sectional curvature), harmonic maps and harmonic maps with free boundary data may exhibit discontinuities. For harmonic maps defined on a domain of dimension at least 3 , free boundary data can have discontinuities on the boundary $[11,17,18]$ and there are even harmonic maps into spheres which are discontinuous everywhere [33]. However, it is possible to establish (partial) regularity for harmonic maps which are minimising (or stationary) with respect to free boundary conditions. This is the primary reason that we consider minimisers.

The methodology we use to prove our theorem is based on existing theories for minimising (free boundary) harmonic maps. Similarly to these theories, our task is divided into two parts. First we prove Hölder continuity, and then that this continuity implies higher regularity. To establish Hölder continuity our main construction will be comparison maps. Such maps were first used in the theory of harmonic maps by Morrey in order to prove interior regularity of energy minimising harmonic maps on two dimensional domains [27]. Schoen and Uhlenbeck later showed that on domains of dimension at least three, minimising harmonic maps are regular away from a set of Hausdorff dimension at most the dimension of the domain minus three [37]; they also used manifold valued comparison maps, which are more difficult to construct on domains of dimension greater than two. The aforementioned results of Hardt and Lin and Duzaar and Steffen for minimising free boundary harmonic maps are established using methods based the constructions of Schoen and Uhlenbeck. We develop a (partial) 
generalisation of a construction of Luckhaus [23] as presented by Simon [39]; the advantage of this construction, at least in existing theories of harmonic maps, is that it can be used to show blow-up limits of sequences of minimisers are again locally minimising. We do not establish this fact here but expect similar results to follow from our constructions. In Sect. 4 we construct comparison maps on a half-cylinder and use these to construct comparison maps on half-balls in $\mathbb{R}_{+}^{m+1}$ centred on $\partial \mathbb{R}_{+}^{m+1}$. We also establish monotonicity of a local, re-scaled version of $E^{\beta}$ and estimates on the mean squared oscillation of the maps we consider via a blow-up argument. The combination of these results is enough to prove continuity. In order to prove Hölder continuity implies higher regularity (away from the singular set), we use a version of harmonic replacement to show $\nabla v \in L^{\infty}$ similarly to [38]. We then establish energy decay for the derivatives of $v$ via another blow-up argument which yields control of the mean squared oscillation of the derivatives of $v$ tangential to $\mathcal{O}$.

To conclude our discussion, we compare the notions of intrinsic and extrinsic fractional harmonic maps and remark on known results regarding their regularity. The first to consider such maps were Da Lio and Rivière [8]. They analysed critical points $u$ of the energy $\left\|(-\Delta)^{\frac{1}{4}} u\right\|_{L^{2}\left(\partial \mathbb{R}_{+}^{2}\right)}^{2}$ in $\dot{H}^{\frac{1}{2}}$ under the constraint that $u$ takes values in the unit $n-1$ sphere $\mathbb{S}^{n-1}$ almost everywhere. In other words, they considered critical points of the functional

$$
L(u)=\inf \left\{E^{0}(v):\left.v\right|_{\partial \mathbb{R}_{+}^{2}}=u, v \in \dot{W}^{1,2}\left(\mathbb{R}_{+}^{2} ; \mathbb{R}^{n}\right), u(x) \in \mathbb{S}^{n-1} \text { for a.e } x \in \partial \mathbb{R}_{+}^{2}\right\} .
$$

The maps Da Lio and Rivière considered fall into a larger class of maps known as extrinsic (fractional) harmonic maps. In contrast to intrinsic fractional harmonic maps, the energies for which extrinsic fractional harmonic maps are critical points depend upon the choice of embedding of the target manifold $N$ into some Euclidean space. Moser [30] proposed to remove this dependence in the energy $L$ so that only the geometry of $N$ plays a role in the theory. In particular, to define $I^{0}(u)$ he minimised $E^{0}(v)$ over extensions $v: \mathbb{R}_{+}^{m+1} \rightarrow N$ with $\left.v\right|_{\mathcal{O}}=u$ as opposed to minimising over extensions $v: \mathbb{R}_{+}^{m+1} \rightarrow \mathbb{R}^{n}$ with $\left.v\right|_{\mathcal{O}}=u$ as is done to define $L(u)$ (for $\mathcal{O}=\partial \mathbb{R}_{+}^{2}$ ). This choice results in another fundamental difference between intrinsic and extrinsic fractional harmonic maps which we illustrate for the power $\frac{1}{2}$ (when $\beta=0$ ). Observe that any $v: \mathbb{R}_{+}^{2} \rightarrow \mathbb{R}^{n}$ for which $L(u)=E^{0}(v)$ satisfies $\Delta v=0$ in $\mathbb{R}_{+}^{2}$ and is hence smooth on $\mathbb{R}_{+}^{2}$ ( $\Delta$ is the usual Laplace operator on Euclidean space). In contrast, any $v: \mathbb{R}_{+}^{m+1} \rightarrow N$ for which $I^{0}(u)=E^{0}(v)$ is a (weakly) harmonic map from $\mathbb{R}_{+}^{m+1}$ to $N$ and satisfies

$$
\Delta v+A(v)(\nabla v, \nabla v)=0 \text { in } \mathbb{R}_{+}^{m+1} .
$$

Unlike solutions of $\Delta v=0$, solutions of (1.5) may have singularities in $\mathbb{R}_{+}^{m+1}$ in general. We conclude extrinsic $\frac{1}{2}$-harmonic maps always have a smooth extension to $\mathbb{R}_{+}^{m+1}$ but intrinsic $\frac{1}{2}$-harmonic maps need not. The same is true for all powers in $(0,1)$.

The regularity theory for extrinsic $\frac{1}{2}$-harmonic maps has been extended to a range of fractional powers for maps into general target manifolds $N$ by Da Lio et al. $[6,7,9,35,36]$ etc, as well as Millot and Sire [25] for the power $\frac{1}{2}$. Millot-Sire and Yu have also considered the regularity of extrinsic minimising fractional harmonic maps defined on the real line for powers in $\left(0, \frac{1}{2}\right)$ [26]. The methods Da Lio, Rivière and Schikorra used to obtain regularity take advantage of compensation phenomena, namely higher than expected regularity which can be derived from the Euler-Lagrange equations for the energies they considered. The higher regularity is revealed using a well chosen gauge transformation, in analogy to techniques used in the theory of harmonic maps. One of the earliest applications of this technique 
was used by Hélein to prove regularity for harmonic maps from Riemannian surfaces into spheres [20]. Millot, Sire and Yu instead consider the extensions of fractional harmonic maps and exploit their connection with free boundary problems for harmonic maps, a method first applied by Moser in the intrinsic case for $\frac{1}{2}$-harmonic maps to obtain (partial) regularity. To our knowledge, aside from the results we establish in this article, the only results regarding intrinsic fractional harmonic maps are those of Moser [30].

\section{Preliminaries}

The Euler-Lagrange equations for $E^{\beta}$ at critical points $v: \mathbb{R}_{+}^{m+1} \rightarrow N$ are semi-linear with leading order term $\operatorname{div}\left(x_{m+1}^{\beta} \nabla v\right)$. We will require Sobolev spaces suited to the analysis of solutions of such equations, as well as the associated linear equation $\operatorname{div}\left(x_{m+1}^{\beta} \nabla v\right)=0$. For $\beta \neq 0$ the coefficient $x_{m+1}^{\beta}$ degenerates or becomes singular on $\partial \mathbb{R}_{+}^{m+1}$ depending on the sign of $\beta$ and the theory of uniformly elliptic second order partial differential equations does not apply on sets overlapping $\partial \mathbb{R}_{+}^{m+1}$. Viewing the coefficient $x_{m+1}^{\beta}$ as a weighting (density) of the Lebesgue measure $\mathrm{d} x$, we may instead appeal to the theory of weighted second order degenerate elliptic equations. We recall and define the function spaces, and some of their analytical properties, necessary for our analysis and then record properties of solutions to $\operatorname{div}\left(x_{m+1}^{\beta} \nabla v\right)=0$ which we will require for the study of solutions of the Euler-Lagrange equations of $E^{\beta}$.

For every $\beta \in(-1,1)$, there exists $C>0$ such that $\frac{1}{\int_{B} \mathrm{~d} x} \int_{B}\left|x_{m+1}\right|^{\beta} \mathrm{d} x \frac{1}{\int_{B} \mathrm{~d} x} \int_{B}\left|x_{m+1}\right|^{-\beta}$ $\mathrm{d} x \leq C$ for every ball $B \subset \mathbb{R}^{m+1}$, where $\mathrm{d} x$ is the Lebesgue measure on $\mathbb{R}^{m+1}$. Hence $\left|x_{m+1}\right|^{\beta}$ is a weight of Muckenhoupt class $A_{2}$, see [19] for an overview of these weights. Every such weight is canonically associated to corresponding Sobolev and Lebesgue spaces. Let $\Omega \subset \mathbb{R}^{m+1}$. Define

$$
L_{\beta}^{2}\left(\Omega ; \mathbb{R}^{n}\right)=\left\{f: \Omega \rightarrow \mathbb{R}^{n}: f \text { is measurable, } \int_{\Omega}|f|^{2}\left|x_{m+1}\right|^{\beta} \mathrm{d} x<\infty\right\}
$$

which is a Hilbert space, see [5, Theorem 3.4.1], where the inner product of $f, g \in L_{\beta}^{2}\left(\Omega ; \mathbb{R}^{n}\right)$ is given by $\langle f, g\rangle_{L_{\beta}^{2}\left(\Omega ; \mathbb{R}^{n}\right)}=\int_{\Omega}\langle f, g\rangle\left|x_{m+1}\right|^{\beta} \mathrm{d} x$ where $\langle f, g\rangle$ is the inner product of $f$ and $g$ in $\mathbb{R}^{n}$. Define

$$
W_{\beta}^{1,2}\left(\Omega ; \mathbb{R}^{n}\right)=\left\{v: \Omega \rightarrow \mathbb{R}^{n}: v, \partial_{i} v \in L_{\beta}^{2}\left(\Omega ; \mathbb{R}^{n}\right) \text { for } i=1, \ldots, m+1\right\}
$$

where $\partial_{i} v$ denotes the weak partial derivative of $v$ with respect to $x_{i}$. Proposition 2.1.2 of [40] guarantees that $W_{\beta}^{1,2}\left(\Omega ; \mathbb{R}^{n}\right)$ is a Hilbert space with the inner product $\langle v, w\rangle_{W_{\beta}^{1,2}\left(\Omega ; \mathbb{R}^{n}\right)}=$ $\int_{\Omega}\langle v, w\rangle\left|x_{m+1}\right|^{\beta} \mathrm{d} x+\int_{\Omega}\langle\nabla u, \nabla v\rangle\left|x_{m+1}\right|^{\beta} \mathrm{d} x$ for $v, w \in W_{\beta}^{1,2}\left(\Omega ; \mathbb{R}^{n}\right)$, where we write $\langle\nabla v, \nabla w\rangle=\sum_{i=1}^{m+1}\left\langle\partial_{i} v, \partial_{i} w\right\rangle$. We also define the Hilbert space $W_{\beta, 0}^{1,2}\left(\Omega ; \mathbb{R}^{n}\right)$ as the closure of $C_{0}^{\infty}\left(\Omega ; \mathbb{R}^{n}\right)$ in $W_{\beta}^{1,2}\left(\Omega ; \mathbb{R}^{n}\right)$ with respect to the norm induced by the inner product on $W_{\beta}^{1,2}\left(\Omega ; \mathbb{R}^{n}\right)$. When $\beta=0$ we omit the subscript $\beta$ from the preceding notation.

It is worth noting that, for every $\beta \in(-1,1) \backslash\{0\}$, approximation by smooth functions in $L_{\beta}^{2}\left(\Omega ; \mathbb{R}^{n}\right)$ and $W_{\beta}^{1,2}\left(\Omega ; \mathbb{R}^{n}\right)$ works in the same way as for the spaces $L^{2}\left(\Omega ; \mathbb{R}^{n}\right)$ and $W^{1,2}\left(\Omega ; \mathbb{R}^{n}\right)$. The details of this process are given in Theorem 2.1.4 and Corollary 2.1.5 in [40]. The spaces $W_{\beta}^{1,2}, W_{\beta, 0}^{1,2}$ and $L_{\beta}^{2}$ also have essentially the same analytical properties, such as completeness, reflexivity etc. as the unweighted spaces $W^{1,2}, W_{0}^{1,2}$ and $L^{2}$ respectively. 
Moreover, since $\left|x_{m+1}\right|^{\beta}$ is an $A_{2}$ weight, many inequalities that hold for the spaces $W^{1, p}$, such as the Poincaré inequality, have counterparts for functions in $W_{\beta}^{1,2}$ [19].

We will need to refer to the relationship between $W_{\beta}^{1,2}$ and $W^{1, p}$ and record it in the following Lemma.

Lemma 2.1 Let $\beta \in(-1,1)$ and suppose $\Omega \subset \mathbb{R}_{+}^{m+1}$ is open and bounded.

1. If $\bar{\Omega} \subset \mathbb{R}_{+}^{m+1}$ then $W_{\beta}^{1,2}\left(\Omega ; \mathbb{R}^{n}\right)=W^{1,2}\left(\Omega ; \mathbb{R}^{n}\right)$.

2. If $\beta \leq 0$ then $W_{\beta}^{1,2}\left(\Omega ; \mathbb{R}^{n}\right) \subset W^{1,2}\left(\Omega ; \mathbb{R}^{n}\right)$.

3. If $\beta>0$ then $W_{\beta}^{1,2}\left(\Omega ; \mathbb{R}^{n}\right) \subset W^{1, p}\left(\Omega ; \mathbb{R}^{n}\right)$ for every $1 \leq p<\frac{2}{1+\beta}$.

Proof Part 1 follows as in this case the norms on $W_{\beta}^{1,2}\left(\Omega ; \mathbb{R}^{n}\right)$ and $W^{1,2}\left(\Omega ; \mathbb{R}^{n}\right)$ are equivalent. Part 2 follows as in this case the $W^{1,2}\left(\Omega ; \mathbb{R}^{n}\right)$ norm is dominated by a constant multiple (depending on $\Omega$ ) of the $W_{\beta}^{1,2}\left(\Omega ; \mathbb{R}^{n}\right)$ norm. Part 3 follows from Hölder's inequality.

\subsection{Weighted homogeneous Sobolev spaces}

Consider the Dirichlet energies

$$
E^{\beta}(v)=\frac{1}{2} \int_{\mathbb{R}_{+}^{m+1}} x_{m+1}^{\beta}|\nabla v|^{2} \mathrm{~d} x
$$

where $\beta \in(-1,1)$, which are well defined on

$$
\mathcal{D}_{+}\left(\mathbb{R}_{+}^{m+1} ; \mathbb{R}^{n}\right):=\left\{\phi|\phi=f|_{\mathbb{R}_{+}^{m+1}} \text { for some } f \in C_{0}^{\infty}\left(\mathbb{R}^{m+1} ; \mathbb{R}^{n}\right)\right\} .
$$

The energy $E^{\beta}$ is naturally associated to the following Sobolev space.

Definition 2.2 Let $\beta \in(-1,1)$. The Weighted Homogeneous Sobolev Space $\dot{W}_{\beta}^{1,2}\left(\mathbb{R}_{+}^{m+1} ; \mathbb{R}^{n}\right)$ is the completion of $\mathcal{D}_{+}\left(\mathbb{R}_{+}^{m+1} ; \mathbb{R}^{n}\right)$ with respect to the metric induced by the square root of $E^{\beta}$.

The elements of $\dot{W}_{\beta}^{1,2}\left(\mathbb{R}_{+}^{m+1} ; \mathbb{R}^{n}\right)$ are, strictly speaking, equivalence classes of Cauchy sequences and it will be necessary to have tangible representatives of these classes which may take values in $N$.

Lemma 2.3 Let $m \in \mathbb{N}$ with $m \geq 2$ and $\Omega$ be an open bounded subset of $\mathbb{R}_{+}^{m+1}$. If $m=2$ let $\beta \in\left(-3^{-1}, 1\right)$ and if $m \geq 3$ let $\beta \in(-1,1)$. Then the restriction operator $I: \mathcal{D}_{+}\left(\mathbb{R}_{+}^{m+1} ; \mathbb{R}^{n}\right) \rightarrow W_{\beta}^{1,2}\left(\Omega ; \mathbb{R}^{n}\right):\left.f \mapsto f\right|_{\Omega}$ extends to a bounded linear operator $I: \dot{W}_{\beta}^{1,2}\left(\mathbb{R}_{+}^{m+1} ; \mathbb{R}^{n}\right) \rightarrow W_{\beta}^{1,2}\left(\Omega ; \mathbb{R}^{n}\right)$. Moreover,

$$
\|I v\|_{W_{\beta}^{1,2}\left(\Omega ; \mathbb{R}^{n}\right)} \leq C\|v\|_{\dot{W}_{\beta}^{1,2}\left(\mathbb{R}_{+}^{m+1} ; \mathbb{R}^{n}\right)}
$$

for every $v \in \dot{W}_{\beta}^{1,2}\left(\mathbb{R}_{+}^{m+1} ; \mathbb{R}^{n}\right)$ where $C$ is a positive constant depending on $m$ and $\Omega$.

Proof It suffices to establish that (2.2) holds for all $\phi \in \mathcal{D}_{+}\left(\mathbb{R}_{+}^{m+1} ; \mathbb{R}^{n}\right)$ as this space is dense in $\dot{W}_{\beta}^{1,2}\left(\mathbb{R}_{+}^{m+1} ; \mathbb{R}^{n}\right)$ and $W_{\beta}^{1,2}\left(\Omega ; \mathbb{R}^{n}\right)$ is a Banach Space. Let $\phi \in \mathcal{D}_{+}\left(\mathbb{R}_{+}^{m+1} ; \mathbb{R}^{n}\right)$. By definition we have $\|\nabla \phi\|_{L_{\beta}^{2}\left(\Omega ; \mathbb{R}^{n}\right)}^{2} \leq\|\phi\|_{\dot{W}_{\beta}^{1,2}\left(\mathbb{R}_{+}^{m+1} ; \mathbb{R}^{n}\right)}^{2}$ so we need to estimate $\|\phi\|_{L_{\beta}^{2}\left(\Omega ; \mathbb{R}^{n}\right)}^{2}$ in terms of $\|\phi\|_{\dot{W}_{\beta}^{1,2}\left(\mathbb{R}_{+}^{m+1} ; \mathbb{R}^{n}\right)}^{2}$. 
Suppose $m \geq 3$. We have $\phi\left(\cdot, x_{m+1}\right) \in W^{1,2}\left(\mathbb{R}^{m} ; \mathbb{R}^{n}\right)$ for every $x_{m+1} \in[0, \infty)$. Let $\nabla^{\prime}$ denote the derivative of $\phi$ with respect to $x^{\prime}$ for $\left(x^{\prime}, x_{m+1}\right) \in \mathbb{R}^{m} \times[0, \infty)$, define $a=\inf _{\Omega}\left(x_{m+1}\right)$ and $b=\sup _{\Omega}\left(x_{m+1}\right)$ and set $l\left(x_{m+1}\right)=\Omega \cap\left(\mathbb{R}^{m} \times\left\{x_{m+1}\right\}\right)$. We apply Fubini's Theorem, together with Hölder's inequality, for conjugate exponents $\frac{m}{m-2}$ and $\frac{m}{2}$, and the Sobolev inequality in $\mathbb{R}^{m}$ to see that

$$
\begin{aligned}
\int_{\Omega} x_{m+1}^{\beta}|\phi|^{2} \mathrm{~d} x & =\int_{a}^{b} x_{m+1}^{\beta} \int_{l\left(x_{m+1}\right)}|\phi|^{2} \mathrm{~d} x^{\prime} \mathrm{d} x_{m+1} \\
& \leq C(\operatorname{diam}(\Omega))^{2} \int_{a}^{b} x_{m+1}^{\beta}\left(\int_{\mathbb{R}^{m}}|\phi|^{\frac{2 m}{m-2}} \mathrm{~d} x^{\prime}\right)^{\frac{m-2}{m}} \mathrm{~d} x_{m+1} \\
& \leq C(\operatorname{diam}(\Omega))^{2} \int_{a}^{b} x_{m+1}^{\beta} \int_{\mathbb{R}^{m}}\left|\nabla^{\prime} \phi\right|^{2} \mathrm{~d} x^{\prime} \mathrm{d} x_{m+1} \\
& \leq C(\operatorname{diam}(\Omega))^{2} \int_{\mathbb{R}_{+}^{m+1}} x_{m+1}^{\beta}\left|\nabla^{\prime} \phi\right|^{2} \mathrm{~d} x
\end{aligned}
$$

We conclude that $\|\phi\|_{W_{\beta}^{1,2}\left(\Omega ; \mathbb{R}^{n}\right)}^{2} \leq C\|\phi\|_{\dot{W}_{\beta}^{1,2}\left(\mathbb{R}_{+}^{m+1} ; \mathbb{R}^{n}\right)}^{2}$.

In order to apply the preceding method of proof when $m=2$ we would need to use the Sobolev Embedding Theorem on $\mathbb{R}$. However, in this case the theorem yields $W^{1,2}(\mathbb{R}) \hookrightarrow$ $L^{\infty}(\mathbb{R})$ and our previous method of proof is no longer viable. However, provided $\beta>-3^{-1}$, Corollary 2 in Section 2.1 .7 of [24] implies that for every $\phi \in C_{0}^{\infty}\left(\mathbb{R}^{3} ; \mathbb{R}^{n}\right)$, and therefore by approximation every $\phi \in W_{\beta}^{1,2}\left(\mathbb{R}^{3} ; \mathbb{R}^{n}\right)$, we have

$$
\left(\int_{\mathbb{R}^{3}}\left|x_{3}\right|^{3 \beta}|\phi|^{6} \mathrm{~d} x\right)^{\frac{1}{3}} \leq C \int_{\mathbb{R}^{3}}\left|x_{3}\right|^{\beta}|\nabla \phi|^{2} \mathrm{~d} x .
$$

The even reflection with respect to $\partial \mathbb{R}_{+}^{m+1}$, denoted $\tilde{\phi}$, of a $\phi \in \mathcal{D}_{+}\left(\mathbb{R}_{+}^{m+1} ; \mathbb{R}^{n}\right)$ is in $W_{\beta}^{1,2}\left(\mathbb{R}^{3} ; \mathbb{R}^{n}\right)$ and hence, applying Hölder's inequality, we find

$$
\begin{aligned}
\int_{\Omega} x_{3}^{\beta}|\phi|^{2} \mathrm{~d} x & \leq|\Omega|^{\frac{2}{3}}\left(\int_{\mathbb{R}^{3}}\left|x_{3}\right|^{3 \beta}|\tilde{\phi}|^{6} \mathrm{~d} x\right)^{\frac{1}{3}} \leq C|\Omega|^{\frac{2}{3}} \int_{\mathbb{R}^{3}}\left|x_{3}\right|^{\beta}|\nabla \tilde{\phi}|^{2} \mathrm{~d} x \\
& =2 C|\Omega|^{\frac{2}{3}} \int_{\mathbb{R}_{+}^{3}} x_{3}^{\beta}|\nabla \phi|^{2} \mathrm{~d} x
\end{aligned}
$$

for every $\phi \in \mathcal{D}_{+}\left(\mathbb{R}_{+}^{3} ; \mathbb{R}^{n}\right)$. We again conclude $\|\phi\|_{W_{\beta}^{1,2}\left(\Omega ; \mathbb{R}^{n}\right)}^{2} \leq C\|\phi\|_{\dot{W}_{\beta}^{1,2}\left(\mathbb{R}_{+}^{m+1} ; \mathbb{R}^{n}\right)}^{2}$.

Remark 2.4 We can further embedd $\dot{W}_{\beta}^{1,2}\left(\mathbb{R}_{+}^{m+1} ; \mathbb{R}^{n}\right)$ into $W^{1, p}\left(\Omega ; \mathbb{R}^{n}\right)$, where $p$ depends on $\beta$, for open bounded $\Omega \subset \mathbb{R}_{+}^{m+1}$ using Lemma 2.1 .

\subsection{Compactness of the embedding $W_{\beta}^{1,2} \hookrightarrow L_{\beta}^{2}$}

We will require an analogue of the compact embedding $W^{1,2} \hookrightarrow L^{2}$ in order to analyse rescaled limits of bounded sequences of Sobolev functions. Away from the boundary, that is, for $\Omega$ with $\bar{\Omega} \subset \mathbb{R}_{+}^{m+1}$, the compactness of the inclusion $W^{1,2} \hookrightarrow L^{2}$ yields the compactness of the inclusion $W_{\beta}^{1,2} \hookrightarrow L_{\beta}^{2}$ in view of Lemma 2.1. We have not been able to find a proof of compactness near $\partial \mathbb{R}_{+}^{m+1}$ in the literature so present one for completeness. Let 
$B_{r}(y)=\left\{x \in \mathbb{R}^{m+1}:|x-y|<r\right\}$ and $Q_{r}(y)=\left\{x \in \mathbb{R}^{m+1}:\left|x_{i}-y_{i}\right|<r, i=1, \ldots, m+1\right\}$ and define $B_{r}^{+}(y)=B_{r}(y) \cap \mathbb{R}_{+}^{m+1}$ and $Q_{r}^{+}(y)=Q_{r}(y) \cap \mathbb{R}_{+}^{m+1}$ for $y \in \partial \mathbb{R}_{+}^{m+1}$.

Lemma 2.5 Let $r>0, y \in \partial \mathbb{R}_{+}^{m+1}$ and suppose $\left(v_{j}\right)_{j \in \mathbb{N}}$ is a sequence in $W_{\beta}^{1,2}\left(\Omega ; \mathbb{R}^{n}\right)$ which satisfies $\sup _{j}\left\|v_{j}\right\|_{W_{\beta}^{1,2}\left(\Omega ; \mathbb{R}^{n}\right)}<\infty$ where $\Omega$ is either $Q_{r}^{+}(y)$ or $B_{r}^{+}(y)$. Then there exists a subsequence $\left(v_{j_{k}}\right)_{k \in \mathbb{N}}$ and $a v \in W_{\beta}^{1,2}\left(\Omega ; \mathbb{R}^{n}\right)$ such that

1. $v_{j_{k}} \rightarrow$ vin $W_{\beta}^{1,2}\left(\Omega ; \mathbb{R}^{n}\right)$

2. $v_{j_{k}} \rightarrow$ vin $L_{\beta}^{2}\left(\Omega ; \mathbb{R}^{n}\right)$

3. $\int_{\Omega} x_{m+1}^{\beta}|\nabla v|^{2} \mathrm{~d} x \leq \liminf _{k \rightarrow \infty} \int_{\Omega} x_{m+1}^{\beta}\left|\nabla v_{j_{k}}\right|^{2} \mathrm{~d} x$.

Proof For $\beta=0$, a proof can be found in [39, Section 1.3, Lemma 1]. Otherwise, statement 1 follows from the weak sequential compactness of the unit ball in a Hilbert space and statement 3 follows from statement 2 and the lower semi-continuity of a Hilbert space norm. Hence, the main task is to prove statement 2. We may assume $r=1$ and $y=0$ since statement 2 is invariant under rescaling and translations with respect to $x_{i}$ for $i=1, \ldots, m$. We further assume that $\Omega=Q_{1}^{+}(0)$ since if $\Omega=B_{1}^{+}(0)$ and the result is true on $Q_{1}^{+}(0)$ then we may compose with the bi-Lipschitz, piecewise $C^{1}$ with piecewise $C^{1}$ inverse map $Q_{1}^{+}(0) \rightarrow B_{1}^{+}(0): x \mapsto \max _{i}\left|x_{i}\right||x|^{-1} x$ and deduce the statement on $B_{1}^{+}(0)$.

Suppose $\left(v_{j}\right)_{j \in \mathbb{N}}$ is a sequence with $v_{j} \in W_{\beta}^{1,2}\left(Q_{1}^{+}(0) ; \mathbb{R}^{n}\right)$ for every $j$ and which satisfies

$$
\sup _{j \in \mathbb{N}}\left\|v_{j}\right\|_{W_{\beta}^{1,2}\left(Q_{1}^{+}(0) ; \mathbb{R}^{n}\right)} \leq M
$$

for some positive constant $M$. Relabelling if necessary, suppose $\left(v_{j}\right)_{j \in \mathbb{N}}$ is also the subsequence which satisfies $v_{j} \rightarrow v$ for $v \in W_{\beta}^{1,2}\left(Q_{1}^{+}(0) ; \mathbb{R}^{n}\right)$. Then

$$
\|v\|_{W_{\beta}^{1,2}\left(Q_{1}^{+}(0) ; \mathbb{R}^{n}\right)} \leq M .
$$

Let $Q^{\prime}=(-1,1)^{m}$, define $Q_{i}=\left\{\left(x^{\prime}, x_{m+1}\right) \in Q_{1}^{+}(0):(i+1)^{-1}<x_{m+1} \leq 1\right\}$ for $i \in \mathbb{N}$ and let $\hat{Q}_{i}=Q_{1}^{+}(0) \backslash Q_{i}=Q^{\prime} \times\left[0,(i+1)^{-1}\right]$. In view of (2.3), we have $\sup _{j \in \mathbb{N}}\left\|v_{j}\right\|_{W_{\beta}^{1,2}\left(Q_{i} ; \mathbb{R}^{n}\right)} \leq M$ for each $i \in \mathbb{N}$. Hence, using the compactness of the embed$\operatorname{ding} W_{\beta}^{1,2}\left(Q_{1} ; \mathbb{R}^{n}\right) \hookrightarrow L_{\beta}^{2}\left(Q_{1} ; \mathbb{R}^{n}\right)$, we find a $\tilde{v} \in W_{\beta}^{1,2}\left(Q_{1} ; \mathbb{R}^{n}\right)$ and a subsequence, which we denote $\left(v_{j}\right)_{j \in \Lambda_{1}}$ for an infinite set $\Lambda_{1} \subset \mathbb{N}$, which satisfies $v_{j} \rightarrow \tilde{v}$ in $W_{\beta}^{1,2}\left(Q_{1} ; \mathbb{R}^{n}\right)$, $v_{j} \rightarrow \tilde{v}$ in $L_{\beta}^{2}\left(Q_{1} ; \mathbb{R}^{n}\right)$ and almost everywhere as $j \rightarrow \infty$ with $j \in \Lambda_{1}$. Notice that $\left(v_{j}\right)_{j \in \Lambda_{1}}$ converges weakly to $v$ in $W_{\beta}^{1,2}\left(Q_{1} ; \mathbb{R}^{n}\right)$ because $\left(v_{j}\right)_{j \in \mathbb{N}}$ does and so, by the uniqueness of weak limits, we deduce $\tilde{v}=v$ in $Q_{1}$. Hence $v_{j} \rightarrow v$ in $L_{\beta}^{2}\left(Q_{1} ; \mathbb{R}^{n}\right)$ and almost everywhere as $j \rightarrow \infty$ as well. Repeating this process inductively for every $i \in \mathbb{N}$, we obtain sequences $\left(v_{j}\right)_{j \in \Lambda_{i}}$ with $\Lambda_{i+1} \subset \Lambda_{i}$ such that $\left(v_{j}\right)_{j \in \Lambda_{i}}$ converges to $v$ in $L_{\beta}^{2}\left(Q_{i} ; \mathbb{R}^{n}\right)$ and almost everywhere in $Q_{i}$. Hence we can choose an increasing sequence of numbers $\left(k_{i}\right)_{i \in \mathbb{N}}$ with $k_{i} \in \Lambda_{i}$ such that

$$
\int_{Q_{i}} x_{m+1}^{\beta}\left|v_{k}-v\right|^{2} \mathrm{~d} x<\frac{\int_{\frac{1}{i+1}}^{\frac{1}{i}} x_{m+1}^{\beta} \mathrm{d} x_{m+1}}{2^{i}} \leq \frac{1}{i^{2+\beta} 2^{i}}
$$

for $k \geq k_{i}$. Then the sequence $\left(v_{k_{i}}\right)_{i \in \mathbb{N}}$ converges to $v$ almost everywhere in $Q_{1}^{+}(0)$ and in $L_{\beta}^{2}\left(Q_{k} ; \mathbb{R}^{n}\right)$ for all $k \in \mathbb{N}$ as $i \rightarrow \infty$. Observe that

$$
\int_{Q_{1}^{+}(0)} x_{m+1}^{\beta}\left|v_{k_{i}}-v\right|^{2} \mathrm{~d} x=\int_{\hat{Q}_{i}} x_{m+1}^{\beta}\left|v_{k_{i}}-v\right|^{2} \mathrm{~d} x+\int_{Q_{i}} x_{m+1}^{\beta}\left|v_{k_{i}}-v\right|^{2} \mathrm{~d} x .
$$


By (2.5) we have $\int_{Q_{i}} x_{m+1}^{\beta}\left|v_{k_{i}}-v\right|^{2} \mathrm{~d} x<\frac{1}{i^{2+\beta} 2^{i}} \rightarrow 0$ as $i \rightarrow \infty$ so we consider the remaining term in (2.6). Using Chebychev's inequality combined with Fubini's Theorem, we may choose $c_{i} \in\left((i+1)^{-1}, i^{-1}\right)$ such that

$$
\int_{Q^{\prime}}\left|v_{k_{i}}\left(x^{\prime}, c_{i}\right)-v\left(x^{\prime}, c_{i}\right)\right|^{2} \mathrm{~d} x^{\prime} \leq \frac{1}{\left(\int_{\frac{1}{i+1}}^{\frac{1}{i}} x_{m+1}^{\beta} \mathrm{d} x_{m+1}\right)} \int_{\frac{1}{i+1}}^{\frac{1}{i}} \int_{Q^{\prime}} x_{m+1}^{\beta}\left|v_{k_{i}}-v\right|^{2} \mathrm{~d} x .
$$

Now for each $i \in \mathbb{N}$, we calculate

$$
\begin{aligned}
\int_{\hat{Q}_{i}} x_{m+1}^{\beta}\left|v_{k_{i}}-v\right|^{2} \mathrm{~d} x \leq & 4 \int_{\hat{Q}_{i}} x_{m+1}^{\beta}\left|v_{k_{i}}-v_{k_{i}}\left(x^{\prime}, c_{i}\right)\right|^{2} \mathrm{~d} x+4 \int_{\hat{Q}_{i}} x_{m+1}^{\beta}\left|v-v\left(x^{\prime}, c_{i}\right)\right|^{2} \mathrm{~d} x \\
& +4 \int_{\hat{Q}_{i}} x_{m+1}^{\beta}\left|v_{k_{i}}\left(x^{\prime}, c_{i}\right)-v\left(x^{\prime}, c_{i}\right)\right|^{2} \mathrm{~d} x .
\end{aligned}
$$

We apply Hölder's inequality and (2.4) to see that

$$
\begin{aligned}
\int_{\hat{Q}_{i}} x_{m+1}^{\beta}\left|v\left(x^{\prime}, x_{m+1}\right)-v\left(x^{\prime}, c_{i}\right)\right|^{2} \mathrm{~d} x & =\int_{\hat{Q}_{i}} x_{m+1}^{\beta}\left|\int_{x_{m+1}}^{c_{i}} s^{-\frac{\beta}{2}} s^{\frac{\beta}{2}} \partial_{m+1} v\left(x^{\prime}, s\right) \mathrm{d} s\right|^{2} \mathrm{~d} x \\
& \leq \frac{c_{i}^{1-\beta}}{1-\beta^{2}} c_{i}^{1+\beta} \int_{0}^{c_{i}} \int_{Q^{\prime}} x_{m+1}^{\beta}\left|\partial_{m+1} v\right|^{2} \mathrm{~d} x \\
& \leq \frac{1}{1-\beta^{2}} \frac{1}{i^{2}} M^{2}
\end{aligned}
$$

The bound for the integral on the left hand side of (2.9) but with $v$ replaced by $v_{k_{i}}$ is identical. We apply (2.7) followed by (2.5) to see that

$$
\begin{aligned}
\int_{\hat{Q}_{i}} x_{m+1}^{\beta}\left|v_{k_{i}}\left(x^{\prime}, c_{i}\right)-v\left(x^{\prime}, c_{i}\right)\right|^{2} \mathrm{~d} x & \leq \frac{\int_{0}^{\frac{1}{i+1}} x_{m+1}^{\beta} \mathrm{d} x_{m+1}}{\int_{\frac{1}{i+1}}^{\frac{1}{i}} x_{m+1}^{\beta} \mathrm{d} x_{m+1}} \int_{\frac{1}{i+1}}^{\frac{1}{i}} \int_{Q^{\prime}} x_{m+1}^{\beta}\left|v_{k_{i}}-v\right|^{2} \mathrm{~d} x \\
& \leq \frac{1}{(1+\beta) i^{1+\beta} 2^{i}}
\end{aligned}
$$

Finally we combine (2.5), (2.6), (2.8), (2.9) and (2.10) and let $i \rightarrow \infty$ to conclude the proof.

Remark 2.6 The method of proof of Lemma 2.5 is also valid for $\Omega$ of the form $\Omega=\mathcal{O} \times[0, r]$ for $r>0$ and $\mathcal{O} \subset \partial \mathbb{R}_{+}^{m+1}$.

\subsection{Energy decay for a linear Neumann-type problem}

When examining the limit of re-scaled sequences of Sobolev functions, as in the proof of Lemma 4.18, we will obtain a weak solution of the Neumann-type problem

$$
\operatorname{div}\left(x_{m+1}^{\beta} \nabla v\right)=0 \text { in } B_{R}^{+}\left(x_{0}\right) \text { and } x_{m+1}^{\beta} \partial_{m+1} v=0 \text { in } \partial B_{R}^{+}\left(x_{0}\right) \cap \partial \mathbb{R}_{+}^{m+1},
$$

for some $R>0$ and $x_{0} \in \partial \mathbb{R}_{+}^{m+1}$. The rate of decay of the re-scaled energy of such solutions on concentric half-balls centred at $x_{0}$ will play a role in the proof of the aforementioned lemma and we estimate this decay here. 
Lemma 2.7 Let $\beta \in(-1,1), x_{0} \in \partial \mathbb{R}_{+}^{m+1}$ and suppose $v \in W_{\beta}^{1,2}\left(B_{R}^{+}\left(x_{0}\right) ; \mathbb{R}^{n}\right)$ satisfies

$$
\int_{B_{R}^{+}\left(x_{0}\right)} x_{m+1}^{\beta}\langle\nabla v, \nabla \psi\rangle \mathrm{d} x=0
$$

for every $\psi \in C_{0}^{\infty}\left(B_{R}\left(x_{0}\right) ; \mathbb{R}^{n}\right)$. There exists a $\gamma=\gamma(m, \beta) \in(0,1)$ and a positive constant $C$ such that

$$
\left(\frac{r}{2}\right)^{1-m-\beta} \int_{B_{\frac{r}{2}}^{+}\left(x_{0}\right)} x_{m+1}^{\beta}|\nabla v|^{2} \mathrm{~d} x \leq C r^{2 \gamma}
$$

for every $r \leq \frac{R}{2}$.

Proof The even reflection of $v$ in $\partial \mathbb{R}_{+}^{m+1}$, which we do not relabel, belongs to $W_{\beta}^{1,2}\left(B_{R}\left(x_{0}\right)\right.$; $\mathbb{R}^{n}$ ) and satisfies

$$
\int_{B_{R}\left(x_{0}\right)}\left|x_{m+1}\right|^{\beta}\langle\nabla v, \nabla \psi\rangle \mathrm{d} x=0
$$

for every $\psi \in C_{0}^{\infty}\left(B_{R}\left(x_{0}\right) ; \mathbb{R}^{n}\right)$. A result of Fabes et al., see [13, Theorem 2.3.12], implies the local Hölder continuity of $v$ in $B_{R}\left(x_{0}\right)$. In particular, there exists a constant $C$ such that

$$
|v(x)-v(y)| \leq C|x-y|^{\gamma}
$$

for some $\gamma \in(0,1)$ and every $x, y \in B_{\frac{R}{2}}\left(x_{0}\right)$.

By approximation, (2.14) holds for every $\psi \in W_{\beta, 0}^{1,2}\left(B_{R}\left(x_{0}\right) ; \mathbb{R}^{n}\right)$. Let $\eta \in C_{0}^{\infty}\left(B_{r}\right.$ $\left.\left(x_{0}\right) ;[0,1]\right)$ be a cutoff function with $\eta \equiv 1$ in $B_{\frac{r}{2}}\left(x_{0}\right)$ and $|\nabla \eta| \leq \frac{C}{r}$ for a fixed positive $C \geq 2$. We observe that $\phi=\eta^{2}(v-\lambda)$ is an admissible test function for every $\lambda \in \mathbb{R}^{n}$. Testing (2.14) against $\phi$ and using Young's inequality, $a b \leq \delta \frac{a^{2}}{2}+\frac{b^{2}}{2 \delta}$ for $a, b \geq 0$ and $\delta>0$, we see that

$$
\begin{aligned}
\int_{B_{r}\left(x_{0}\right)}\left|x_{m+1}\right|^{\beta} \eta^{2}|\nabla v|^{2} \mathrm{~d} x \leq & \delta \int_{B_{r}\left(x_{0}\right)}\left|x_{m+1}\right|^{\beta} \eta^{2}|\nabla v|^{2} \mathrm{~d} x \\
& +\frac{C}{\delta} \int_{B_{r}\left(x_{0}\right)}\left|x_{m+1}\right|^{\beta}|\nabla \eta|^{2}|v-\lambda|^{2} \mathrm{~d} x .
\end{aligned}
$$

We choose $\delta=\frac{1}{2}$, set $\lambda=v\left(x_{0}\right)$, recall $|\nabla \eta| \leq \frac{C}{r}$ and apply (2.15) to see that

$$
\int_{B_{r}\left(x_{0}\right)}\left|x_{m+1}\right|^{\beta} \eta^{2}|\nabla v|^{2} \mathrm{~d} x \leq C r^{m+\beta-1+2 \gamma}
$$

for another positive $C>0$, independent of $r \leq \frac{R}{2}$. Multiplying $(2.16)$ by $\left(2^{-1} r\right)^{1-m-\beta}$ concludes the proof.

\subsection{Boundary monotonicity formula for the average energy of solutions to the degenerate linear equation}

In order to utilise a version of the method of harmonic replacement, see Lemma 4.23 in Sect. 4.12, we need to know how the average energy of solutions to $\operatorname{div}\left(\left|x_{m+1}\right|^{\beta} \nabla v\right)=0$ behaves on concentric balls. When these balls are centred on $\partial \mathbb{R}_{+}^{m+1}$, we have the following. 
Lemma 2.8 Let $B_{R}\left(x_{0}\right) \subset \mathbb{R}^{m+1}$ with $x_{0} \in \partial \mathbb{R}_{+}^{m+1}$ and $R \leq 1$. Suppose $v \in$ $W_{\beta}^{1,2}\left(B_{R}\left(x_{0}\right) ; \mathbb{R}^{n}\right)$ is a weak solution of $\operatorname{div}\left(\left|x_{m+1}\right|^{\beta} \nabla v\right)=0$ in $B_{R}\left(x_{0}\right)$. If $\beta \in(-1,0]$, or if $\beta \in(0,1)$ and $v$ is symmetric with respect to $\partial \mathbb{R}_{+}^{m+1}$ in $B_{R}\left(x_{0}\right)$, then

$$
s^{-(1+m+\beta)} \int_{B_{s}\left(x_{0}\right)}\left|x_{m+1}\right|^{\beta}|\nabla v|^{2} \mathrm{~d} x \leq r^{-(1+m+\beta)} \int_{B_{r}\left(x_{0}\right)}\left|x_{m+1}\right|^{\beta}|\nabla v|^{2} \mathrm{~d} x
$$

for every $0<s \leq r \leq R$.

Remark 2.9 It appears that Lemma 2.8 is a particular case of Theorem 2.6 of [3]. The function

$$
\tilde{v}\left(x^{\prime}, x_{m+1}\right)=\left\{\begin{array}{ll}
\frac{1}{1-\beta} x_{m+1}^{1-\beta} & \text { if } x_{m+1} \geq 0 \\
-\frac{1}{1-\beta}\left(-x_{m+1}\right)^{1-\beta} & \text { if } x_{m+1}<0
\end{array}=(1-\beta)^{-1} x_{m+1}\left|x_{m+1}\right|^{-\beta}\right.
$$

fulfils the hypothesis of Lemma 2.8, with the exception that it is not symmetric with respect to $\partial \mathbb{R}_{+}^{m+1}$ when $\beta \in(0,1)$. Furthermore, $s^{-(1+m+\beta)} \int_{B_{s}(0)}\left|x_{m+1}\right|^{\beta}|\nabla \tilde{v}|^{2} \mathrm{~d} x=C(m, \beta) s^{-2 \beta}$. It follows that $\tilde{v}$ does not satisfy the conclusion of the lemma (e.g on $B_{1}(0)$ ) when $\beta \in(0,1)$. Hence Lemma 2.8 does not hold in general when $\beta \in(0,1)$. This also potentially affects the aforementioned theorem.

The remainder of this section is predominantly devoted to a proof of Lemma 2.8. We also establish a similar monotonicity formula for balls $B_{R}\left(x_{0}\right)$ with $\left(x_{0}\right)_{m+1} \geq \theta R$ for $\theta \geq 2$ and give explicit dependence on $\theta$. Our method is essentially that used to show the well known monotonicity results for the average energy of sub-harmonic functions, which is based on the observation $\Delta|\nabla v|^{2} \geq 0$ if $\Delta v=0$. When $\beta=0$ our result reduces to the usual growth formula for the average energy of sub-harmonic functions.

Define $v^{*}:=\left|x_{m+1}\right|^{\beta} \partial_{m+1} v$. This function will be integral to our proof of Lemma 2.8 because, as we will see in more detail later, it satisfies $\operatorname{div}\left(\left|x_{m+1}\right|^{-\beta} \nabla v^{*}\right)=0$ when $\operatorname{div}\left(\left|x_{m+1}\right|^{\beta} \nabla v\right)=0$. The fact that if $\operatorname{div}\left(x_{m+1}^{\beta} \nabla v\right)=0$ in $\mathbb{R}_{+}^{m+1}$ then $\operatorname{div}\left(x_{m+1}^{-\beta} \nabla v^{*}\right)=0$ was first observed by Caffarelli and Silvestre [2].

First, we consider the regularity of solutions of $\operatorname{div}\left(\left|x_{m+1}\right|^{\beta} \nabla v\right)=0$; the following regularity results are known and have essentially been obtained in, for example [4]. We give a proof regardless, as an illustration that the method of difference quotients works essentially unchanged in the directions $x_{1}, \ldots, x_{m}$, which we will take advantage of when considering the regularity of manifold valued minimisers of $E^{\beta}$.

Let $i=1, \ldots, m, \Omega \subset \mathbb{R}^{m+1}$ and let $h \in \mathbb{R} \backslash\{0\}$. Define the difference quotient of $v: \Omega \rightarrow \mathbb{R}^{n}$ by $\Delta_{i}^{h} v(x)=h^{-1}\left(v\left(x+h e_{i}\right)-v(x)\right)$ where $e_{i}$ denotes the $i$ th basis vector in $\mathbb{R}^{m+1}$ and $\operatorname{dist}(x, \partial \Omega)<|h|$.

Lemma 2.10 Let $\Omega \subset \mathbb{R}^{m+1}$ be open and $v \in W_{\beta}^{1,2}\left(\Omega ; \mathbb{R}^{n}\right)$. Then for any $i=1, \ldots, m$ we have $\Delta_{i}^{h} v \in L_{\beta}^{2}\left(K ; \mathbb{R}^{n}\right)$ for any compact $K \subset \Omega$, provided $|h|<\operatorname{dist}(K, \partial \Omega)$. In particular,

$$
\int_{K}\left|x_{m+1}\right|^{\beta}\left|\Delta_{i}^{h} v\right|^{2} \mathrm{~d} x \leq \int_{\Omega}\left|x_{m+1}\right|^{\beta}\left|\partial_{i} v\right|^{2} \mathrm{~d} x .
$$

Proof This proof follows the proof of Lemma 7.23 in [15]. We assume that $h \geq 0$, the argument for negative $h$ is analogous. Let $v \in C^{1}\left(\Omega ; \mathbb{R}^{n}\right) \cap W_{\beta}^{1,2}\left(\Omega ; \mathbb{R}^{n}\right)$. Using the notation $K_{h}=\left\{x \in \mathbb{R}^{m+1}: \operatorname{dist}(x, K) \leq h\right\}$ and noting $K_{h} \subset \Omega$, by Fubini's Theorem and the compactness of $K$, for $h$ with $|h|<\operatorname{dist}(K, \partial \Omega)$ and any $i=1, \ldots, m$, we calculate

$$
\int_{K}\left|x_{m+1}\right|^{\beta}\left|\Delta_{i}^{h} v\right|^{2} \mathrm{~d} x \leq \int_{K}\left|x_{m+1}\right|^{\beta} \frac{1}{h} \int_{0}^{h}\left|\partial_{i} v\left(x+t e_{i}\right)\right|^{2} \mathrm{~d} t \mathrm{~d} x
$$




$$
\begin{aligned}
& \leq \frac{1}{h} \int_{0}^{h} \int_{K_{h}}\left|x_{m+1}\right|^{\beta}\left|\partial_{i} v\right|^{2} \mathrm{~d} x \mathrm{~d} t \\
& \leq \int_{\Omega}\left|x_{m+1}\right|^{\beta}\left|\partial_{i} v\right|^{2} \mathrm{~d} x .
\end{aligned}
$$

We deduce the result for $v \in W_{\beta}^{1,2}\left(\Omega ; \mathbb{R}^{n}\right)$ by approximation.

Next we prove a criterion for the existence of weak derivatives in the directions $x_{i}$ for $i=1, \ldots, m$.

Lemma 2.11 Suppose $\Omega \subset \mathbb{R}^{m+1}$ is open and bounded and let $v \in L_{\beta}^{2}\left(\Omega ; \mathbb{R}^{n}\right)$. For any $i=1, \ldots, m$, suppose there exist constants $M>0$ and $\tilde{h}>0$ such that

$$
\int_{K}\left|x_{m+1}\right|^{\beta}\left|\Delta_{i}^{h} v\right|^{2} \mathrm{~d} x \leq M
$$

for every $h \neq 0$ with $|h|<\tilde{h}$ and compact $K \subset \Omega$ with $\operatorname{dist}(K, \partial \Omega)>|h|$. Then the weak derivative $\partial_{i} v$ exists in $\Omega$ and satisfies

$$
\int_{\Omega}\left|x_{m+1}\right|^{\beta}\left|\partial_{i} v\right|^{2} \mathrm{~d} x \leq M
$$

Proof First choose a sequence $\left(h_{k}\right)_{k \in \mathbb{N}}$ with $h_{k} \rightarrow 0$. We discard $h_{k}$ with $\left|h_{k}\right| \geq \tilde{h}$ and reindex to $k \in \mathbb{N}$. Define $v_{i}^{h_{k}}(x)=\Delta_{i}^{h_{k}} v(x)$ when $x \in \Omega$ and $\operatorname{dist}(x, \partial \Omega) \geq 2\left|h_{k}\right|$ and $v_{i}^{h_{k}}=0$ otherwise. It follows that $\left\{v_{i}^{h_{k}}\right\}_{k \in \mathbb{N}}$ is a bounded sequence in $L_{\beta}^{2}\left(\Omega ; \mathbb{R}^{n}\right)$. Hence there is a subsequence, which we index again by $k \in \mathbb{N}$, such that $h_{k} \rightarrow 0$ and $v_{i}^{h_{k}} \rightarrow \tilde{v}_{i}$ weakly in $L_{\beta}^{2}\left(\Omega ; \mathbb{R}^{n}\right)$. Furthermore, this convergence, together with the weak lower semi-continuity of a Hilbert space norm, guarantees that $\int_{\Omega} x_{m+1}^{\beta}\left|\tilde{v}_{i}\right|^{2} \mathrm{~d} x \leq M$. Note that Hölder's inequality implies $L_{\beta}^{2}\left(\Omega ; \mathbb{R}^{n}\right) \subset L^{p}\left(\Omega ; \mathbb{R}^{n}\right)$ for some $p \in(1,2]$ depending on $\beta$. Thus each linear functional on $L^{p}\left(\Omega ; \mathbb{R}^{n}\right)$ restricts to a linear functional on $L_{\beta}^{2}\left(\Omega ; \mathbb{R}^{n}\right)$ and $v_{i}^{h_{k}}$ converges to $\tilde{v}_{i}$ weakly in $L^{p}\left(\Omega ; \mathbb{R}^{n}\right)$. As in the proof of Lemma 7.24 in [15], it follows that $\tilde{v}_{i}$ is the weak derivative $\partial_{i} v$.

We now establish the regularity properties of solutions of $\operatorname{div}\left(\left|x_{m+1}\right|^{\beta} \nabla v\right)=0$ needed for the proof of Lemma 2.8 .

Lemma 2.12 Let $v \in W_{\beta}^{1,2}\left(B_{R}\left(x_{0}\right) ; \mathbb{R}^{n}\right)$ and suppose $v$ is a weak solution of div $\left(\left|x_{m+1}\right|^{\beta} \nabla v\right)$ $=0$ in $B_{R}\left(x_{0}\right)$. For every $r<R$ and $i=1, \ldots, m$ it follows that $\partial_{i} v \in W_{\beta}^{1,2}\left(B_{r}\left(x_{0}\right) ; \mathbb{R}^{n}\right)$, $\partial_{i} v$ is a weak solution of $\operatorname{div}\left(\left|x_{m+1}\right|^{\beta} \nabla v\right)=0$ in $B_{r}\left(x_{0}\right)$ and $\partial_{i} v$ is locally Hölder continuous in $B_{R}\left(x_{0}\right)$. In addition, $v^{*} \in W_{-\beta}^{1,2}\left(B_{r}\left(x_{0}\right) ; \mathbb{R}^{n}\right)$ and $v^{*}$ is a weak solution of $\operatorname{div}\left(\left|x_{m+1}\right|^{-\beta} \nabla v^{*}\right)=0$ in $B_{r}\left(x_{0}\right)$ and $v^{*}$ is locally Hölder continuous in $B_{R}\left(x_{0}\right)$.

Proof Elliptic regularity theory shows that $v$ is smooth in $B_{R}\left(x_{0}\right) \backslash \partial \mathbb{R}_{+}^{m+1}$ [15]. Observe that $v$ satisfies

$$
\int_{B_{R}\left(x_{0}\right)}\left|x_{m+1}\right|^{\beta}\langle\nabla v, \nabla \phi\rangle \mathrm{d} x=0
$$

for every $\phi \in W_{\beta, 0}^{1,2}\left(B_{R}\left(x_{0}\right) ; \mathbb{R}^{n}\right)$ by approximation. Let $r<R$ and choose $\eta \in C_{0}^{\infty}\left(B_{R}\left(x_{0}\right)\right)$ with $\eta \equiv 1$ in $B_{r}\left(x_{0}\right), \eta \equiv 0$ in $B_{R}\left(x_{0}\right) \backslash B_{r+\frac{R-r}{2}}\left(x_{0}\right), 0 \leq \eta \leq 1$ and $|\nabla \eta| \leq \frac{C}{R-r}$. Let $\Delta_{i}^{h} v$ 
be the difference quotient of $v$ for some $i=1, \ldots, m$ and suppose $|h|<\frac{R-r}{4}$. Then $\phi=-\Delta_{i}^{-h}\left(\eta^{2} \Delta_{i}^{h} v\right) \in W_{\beta, 0}^{1,2}\left(B_{R}\left(x_{0}\right) ; \mathbb{R}^{n}\right)$ is an admissible test function for (2.17) and an application of Young's inequality, $a b \leq \delta \frac{a^{2}}{2}+\delta^{-1} \frac{b^{2}}{2}$ for $a, b \geq 0$ and $\delta>0$, together with an integration by parts and Lemma 2.10 implies

$$
\begin{aligned}
\int_{B_{R}\left(x_{0}\right)} \eta^{2}\left|x_{m+1}\right|^{\beta}\left|\nabla \Delta_{i}^{h} v\right|^{2} \mathrm{~d} x= & -\int_{B_{R}\left(x_{0}\right)} 2 \eta\left|x_{m+1}\right|^{\beta}\left\langle\nabla \Delta_{i}^{h} v \cdot \nabla \eta, \Delta_{i}^{h} v\right\rangle \mathrm{d} x \\
\leq & \frac{C}{R-r} \delta \int_{B_{R}\left(x_{0}\right)} \eta^{2}\left|x_{m+1}\right|^{\beta}\left|\nabla \Delta_{i}^{h} v\right|^{2} \mathrm{~d} x \\
& +\frac{C}{R-r} \delta^{-1} \int_{B_{R}\left(x_{0}\right)}\left|x_{m+1}\right|^{\beta}\left|\partial_{i} v\right|^{2} \mathrm{~d} x .
\end{aligned}
$$

Since $\eta \equiv 1$ in $B_{r}\left(x_{0}\right)$, choosing $\delta=\frac{R-r}{2 C}$ we deduce that

$$
\int_{B_{r}\left(x_{0}\right)}\left|x_{m+1}\right|^{\beta}\left|\nabla \Delta_{i}^{h} v\right|^{2} \mathrm{~d} x \leq \frac{C}{(R-r)^{2}} \int_{B_{R}\left(x_{0}\right)}\left|x_{m+1}\right|^{\beta}\left|\partial_{i} v\right|^{2} \mathrm{~d} x .
$$

The right hand side above is independent of $h$ and thus Lemma 2.11 implies the weak derivative $\nabla \partial_{i} v$ exists and is in $L_{\beta}^{2}\left(B_{r}\left(x_{0}\right) ; \mathbb{R}^{(m+1) n}\right)$. Hence $\partial_{i} v \in W_{\beta}^{1,2}\left(B_{r}\left(x_{0}\right) ; \mathbb{R}^{n}\right)$ for every $r<R$. We integrate by parts in (2.17) to see that $\partial_{i} v$ is a weak solution of $\operatorname{div}\left(\left|x_{m+1}\right|^{\beta} \nabla v\right)=0$ in $B_{r}\left(x_{0}\right)$ for every $r<R$. It follows from [13, Theorem 2.3.12] that each $\partial_{i} v$ is locally Hölder continuous in $B_{R}\left(x_{0}\right)$. We inductively deduce that for any multi-index $\alpha^{\prime}$ with $\alpha_{m+1}^{\prime}=0$, $D^{\alpha^{\prime}} v \in W_{\beta}^{1,2}\left(B_{r}\left(x_{0}\right) ; \mathbb{R}^{n}\right)$ is a weak solution of $\operatorname{div}\left(\left|x_{m+1}\right|^{\beta} \nabla D^{\alpha^{\prime}} v\right)=0$ in $B_{r}\left(x_{0}\right)$ for every $r<R$ and $D^{\alpha^{\prime}} v$ is locally Hölder continuous in $B_{R}\left(x_{0}\right)$.

Since $\partial_{i} v \in W_{\beta}^{1,2}\left(B_{r}\left(x_{0}\right) ; \mathbb{R}^{n}\right)$ for every $r<R$ and $i=1, \ldots, m$, we have

$$
\int_{B_{r}\left(x_{0}\right)}\left|x_{m+1}\right|^{-\beta}\left|\partial_{i} v^{*}\right|^{2} \mathrm{~d} x=\int_{B_{r}\left(x_{0}\right)}\left|x_{m+1}\right|^{\beta}\left|\partial_{i} \partial_{m+1} v\right|^{2} \mathrm{~d} x<\infty .
$$

We also have $\Delta^{\prime} v \in W_{\beta}^{1,2}\left(B_{r}\left(x_{0}\right) ; \mathbb{R}^{n}\right)$, where $\Delta^{\prime}$ is the Laplace operator with respect to the variables $x_{1}, \ldots, x_{m}$. Furthermore, as $v$ solves $\operatorname{div}\left(\left|x_{m+1}\right|^{\beta} \nabla v\right)=0$ classically in $B_{R}\left(x_{0}\right) \backslash \partial \mathbb{R}_{+}^{m+1}$, we have $\left|x_{m+1}\right|^{-\beta} \partial_{m+1} v^{*}=-\Delta^{\prime} v \in W_{\beta}^{1,2}\left(B_{r}\left(x_{0}\right) ; \mathbb{R}^{n}\right)$ for every $r<R$. Hence

$$
\int_{B_{r}\left(x_{0}\right)}\left|x_{m+1}\right|^{-\beta}\left|\partial_{m+1} v^{*}\right|^{2} \mathrm{~d} x=\int_{B_{r}\left(x_{0}\right)}\left|x_{m+1}\right|^{\beta}\left|\Delta^{\prime} v\right|^{2} \mathrm{~d} x<\infty .
$$

Together, (2.18) and (2.19) imply $\int_{B_{r}\left(x_{0}\right)}\left|x_{m+1}\right|^{-\beta}\left|\nabla v^{*}\right|^{2} \mathrm{~d} x<\infty$ for every $r<R$. Moreover, we have

$$
\int_{B_{r}\left(x_{0}\right)}\left|x_{m+1}\right|^{-\beta}\left|v^{*}\right|^{2} \mathrm{~d} x=\int_{B_{r}\left(x_{0}\right)}\left|x_{m+1}\right|^{\beta}\left|\partial_{m+1} v\right|^{2} \mathrm{~d} x<\infty,
$$

since $v \in W_{\beta}^{1,2}\left(B_{R}\left(x_{0}\right) ; \mathbb{R}^{n}\right)$. We can directly verify that $\partial_{i} v^{*}, i=1, \ldots, m$, are the weak derivatives of $v^{*}$ and omit the details. Now consider $\partial_{m+1} v^{*}$. Let $\nabla^{\prime}$ denote the gradient operator with respect to the variables $x_{1}, \ldots, x_{m}$ and let $\psi \in C_{0}^{\infty}\left(B_{R}\left(x_{0}\right) ; \mathbb{R}^{n}\right)$. Since $\left|x_{m+1}\right|^{-\beta} \partial_{m+1} v^{*}=-\Delta^{\prime} v \in W_{\beta}^{1,2}\left(B_{r}\left(x_{0}\right) ; \mathbb{R}^{n}\right)$ and $v$ is a weak solution of $\operatorname{div}\left(\left|x_{m+1}\right|^{\beta} \nabla v\right)=0$, we see that

$$
\int_{B_{R}\left(x_{0}\right)}\left\langle\partial_{m+1} v^{*}, \psi\right\rangle \mathrm{d} x=-\int_{B_{R}\left(x_{0}\right)}\left|x_{m+1}\right|^{\beta}\left\langle\Delta^{\prime} v, \psi\right\rangle \mathrm{d} x
$$




$$
\begin{aligned}
& =\int_{B_{R}\left(x_{0}\right)}\left|x_{m+1}\right|^{\beta}\left\langle\nabla^{\prime} v, \nabla^{\prime} \psi\right\rangle \mathrm{d} x \\
& =-\int_{B_{R}\left(x_{0}\right)}\left\langle v^{*}, \partial_{m+1} \psi\right\rangle \mathrm{d} x .
\end{aligned}
$$

It follows that $v^{*} \in W_{-\beta}^{1,2}\left(B_{r}\left(x_{0}\right) ; \mathbb{R}^{n}\right)$ for every $r<R$. In a similar manner we calculate

$$
\begin{aligned}
\int_{B_{R}\left(x_{0}\right)}\left|x_{m+1}\right|^{-\beta}\left\langle\nabla v^{*}, \nabla \psi\right\rangle \mathrm{d} x= & \int_{B_{R}\left(x_{0}\right)}\left|x_{m+1}\right|^{-\beta}\left\langle\partial_{m+1} v^{*}, \partial_{m+1} \psi\right\rangle \mathrm{d} x \\
& +\int_{B_{R}\left(x_{0}\right)}\left|x_{m+1}\right|^{-\beta}\left\langle\nabla^{\prime} v^{*}, \nabla^{\prime} \psi\right\rangle \mathrm{d} x \\
= & -\int_{B_{R}\left(x_{0}\right)}\left\langle\Delta^{\prime} v, \partial_{m+1} \psi\right\rangle \mathrm{d} x+\int_{B_{R}\left(x_{0}\right)}\left\langle\Delta^{\prime} v, \partial_{m+1} \psi\right\rangle \mathrm{d} x \\
= & 0 .
\end{aligned}
$$

Hence $v^{*}$ is a weak solution of $\operatorname{div}\left(\left|x_{m+1}\right|^{\beta} \nabla v^{*}\right)=0$ in $B_{r}\left(x_{0}\right)$ for every $r<R$. It follows from [13, Theorem 2.3.12] that $v^{*}$ is locally Hölder continuous in $B_{R}\left(x_{0}\right)$.

Corollary 2.13 Suppose $v \in W_{\beta}^{1,2}\left(B_{R}\left(x_{0}\right) ; \mathbb{R}^{n}\right)$ and assume $v$ weakly satisfies $\operatorname{div}\left(\left|x_{m+1}\right|^{\beta} \nabla v\right)=0$ in $B_{R}\left(x_{0}\right)$. Then the derivatives $D^{\alpha^{\prime}} v$, where $\alpha^{\prime}$ is a multi-index with $\left(\alpha^{\prime}\right)_{m+1}=0$, are elements of $W_{\beta}^{1,2}\left(B_{r}\left(x_{0}\right) ; \mathbb{R}^{n}\right)$ and weak solutions of $\operatorname{div}\left(\left|x_{m+1}\right|^{\beta} \nabla v\right)=0$ in $B_{r}\left(x_{0}\right)$ for every $r<R$ and are locally Hölder continuous in $B_{R}\left(x_{0}\right)$. Furthermore, the functions $\left(D^{\alpha^{\prime}} v\right)^{*}:=\left|x_{m+1}\right|^{\beta} \partial_{m+1} D^{\alpha^{\prime}} v$ are elements of $W_{-\beta}^{1,2}\left(B_{r}\left(x_{0}\right) ; \mathbb{R}^{n}\right)$ and weak solutions of $\operatorname{div}\left(\left|x_{m+1}\right|^{-\beta} \nabla\left(D^{\alpha^{\prime}} v\right)^{*}\right)=0$ in $B_{r}\left(x_{0}\right)$ for every $r<R$ and are locally Hölder continuous in $B_{R}\left(x_{0}\right)$.

Proof This follows from a direct application of Lemma 2.12.

Next we record a condition on the integral of the normal derivative of Sobolev functions which implies the type of monotonicity we want to establish in Lemma 2.8.

Lemma 2.14 Suppose that $v \in W_{\beta}^{1,2}\left(B_{R}\left(x_{0}\right)\right)$, where $x_{0} \in \partial \mathbb{R}_{+}^{m+1}$, and that

$$
\int_{\partial B_{\rho}\left(x_{0}\right)} v \cdot\left|x_{m+1}\right|^{\beta} \nabla v \mathrm{~d} S(x) \geq 0
$$

for almost every $\rho \in(0, R)$, where $v$ is the outward pointing unit normal on $\partial B_{\rho}\left(x_{0}\right)$, then

$$
s^{-(1+m+\beta)} \int_{B_{s}\left(x_{0}\right)}\left|x_{m+1}\right|^{\beta} v \mathrm{~d} x \leq r^{-(1+m+\beta)} \int_{B_{r}\left(x_{0}\right)}\left|x_{m+1}\right|^{\beta} v \mathrm{~d} x
$$

for every $0<s \leq r \leq R$.

Proof This proof follows the proof of Theorem 2.1 in Section 2 of [15] and the proof of Proposition 2.2 in Section III of [14]. For almost every $0<s \leq r<R$, using Fubini's Theorem, we see that

$$
\begin{aligned}
& r^{-(m+\beta)} \int_{\partial B_{r}\left(x_{0}\right)}\left|x_{m+1}\right|^{\beta} v \mathrm{~d} S(x)-s^{-(m+\beta)} \int_{\partial B_{S}\left(x_{0}\right)}\left|x_{m+1}\right|^{\beta} v \mathrm{~d} S(x) \\
& =\int_{\partial B_{1}(0)}\left|\omega_{m+1}\right|^{\beta} \int_{s}^{r} \frac{\partial}{\partial t} v\left(t \omega+x_{0}\right) \mathrm{d} t \mathrm{~d} \omega
\end{aligned}
$$




$$
\begin{aligned}
& =\int_{s}^{r} t^{-(m+\beta)} \int_{\partial B_{t}\left(x_{0}\right)}\left|x_{m+1}\right|^{\beta} v \cdot \nabla v \mathrm{~d} S(x) \mathrm{d} t \\
& \geq 0 .
\end{aligned}
$$

Define the absolutely continuous function $f(r)=\int_{B_{r}\left(x_{0}\right)}\left|x_{m+1}\right|^{\beta} v \mathrm{~d} x$ for $0 \leq r \leq R$. Using (2.22) we calculate

$$
\begin{aligned}
f(r) & =\int_{0}^{r} f^{\prime}(\rho) \mathrm{d} \rho=\int_{0}^{r} \rho^{m+\beta} \rho^{-(m+\beta)} f^{\prime}(\rho) \mathrm{d} \rho \\
& \leq \int_{0}^{r} \rho^{m+\beta} r^{-(m+\beta)} f^{\prime}(r) \mathrm{d} \rho=\frac{r}{1+m+\beta} f^{\prime}(r)
\end{aligned}
$$

for $0<r<R$. It follows that $\left(r^{-(1+m+\beta)} f(r)\right)^{\prime} \geq 0$ and integrating between $s \leq r \leq R$ completes the proof.

Proof of Lemma 2.8 Note that $v$ is smooth in $B_{R}\left(x_{0}\right) \backslash \partial \mathbb{R}_{+}^{m+1}$. Hence $\operatorname{div}\left(\left|x_{m+1}\right|^{\beta} \nabla \partial_{i} v\right)=0$ and $\operatorname{div}\left(\left|x_{m+1}\right|^{-\beta} \nabla v^{*}\right)=0$ classically in this set. Furthermore, we have

$$
\begin{aligned}
0=\partial_{m+1} \operatorname{div}\left(\left|x_{m+1}\right|^{\beta} \nabla v\right)= & \operatorname{div}\left(\left|x_{m+1}\right|^{\beta} \nabla \partial_{m+1} v\right)+\operatorname{sgn}\left(x_{m+1}\right) \frac{\beta}{\left|x_{m+1}\right|} \operatorname{div}\left(\left|x_{m+1}\right|^{\beta} \nabla v\right) \\
& -\beta\left|x_{m+1}\right|^{\beta-2} \partial_{m+1} v \\
= & \operatorname{div}\left(\left|x_{m+1}\right|^{\beta} \nabla \partial_{m+1} v\right)-\beta\left|x_{m+1}\right|^{\beta-2} \partial_{m+1} v
\end{aligned}
$$

so that $\operatorname{div}\left(\left|x_{m+1}\right|^{\beta} \nabla \partial_{m+1} v\right)=\beta\left|x_{m+1}\right|^{\beta-2} \partial_{m+1} v$ in $B_{R}\left(x_{0}\right) \backslash \partial \mathbb{R}_{+}^{m+1}$. Hence on $B_{R}\left(x_{0}\right) \backslash$ $\partial \mathbb{R}_{+}^{m+1}$ we have

$$
\begin{aligned}
\operatorname{div}\left(\left|x_{m+1}\right|^{\beta} \nabla\left|\partial_{i} v\right|^{2}\right) & =2\left|x_{m+1}\right|^{\beta}\left|\nabla \partial_{i} v\right|^{2}+2\left\langle\partial_{i} v, \operatorname{div}\left(\left|x_{m+1}\right|^{\beta} \nabla \partial_{i} v\right)\right\rangle \geq 0, \\
\operatorname{div}\left(\left|x_{m+1}\right|^{-\beta} \nabla\left|v^{*}\right|^{2}\right) & =2\left|x_{m+1}\right|^{\beta}\left|\nabla v^{*}\right|^{2}+2\left\langle v^{*}, \operatorname{div}\left(\left|x_{m+1}\right|^{-\beta} \nabla v^{*}\right)\right\rangle \geq 0,
\end{aligned}
$$

and, when $\beta \in(0,1)$,

$$
\operatorname{div}\left(\left|x_{m+1}\right|^{\beta} \nabla\left|\partial_{m+1} v\right|^{2}\right)=2\left|x_{m+1}\right|^{\beta}\left|\nabla \partial_{m+1} v\right|^{2}+2 \beta\left|x_{m+1}\right|^{\beta-2}\left|\partial_{m+1} v\right|^{2} \geq 0,
$$

classically where $i=1, \ldots, m$.

Fix $R>r>\varepsilon>0$ and let $B_{r}^{\varepsilon}\left(x_{0}\right)=B_{r}\left(x_{0}\right) \cap\left\{x \in \mathbb{R}^{m+1}:\left|x_{m+1}\right| \geq \varepsilon\right\}$. Using the divergence theorem, we calculate

$$
\begin{aligned}
& 0 \leq \int_{\partial B_{r}\left(x_{0}\right)} \mathbb{1} \frac{}{B_{r}^{\varepsilon}\left(x_{0}\right)}\left|x_{m+1}\right|^{\beta} v \cdot \nabla\left|\partial_{i} v\right|^{2} \mathrm{~d} S(x) \\
& -\int_{B_{\sqrt{r^{2}-\varepsilon^{2}}}} \varepsilon_{\left.x_{0}\right)}^{\beta} e_{m+1} \cdot\left(\nabla\left|\partial_{i} v\right|^{2}\left(x^{\prime}, \varepsilon\right)-\nabla\left|\partial_{i} v\right|^{2}\left(x^{\prime},-\varepsilon\right)\right) \mathrm{d} x^{\prime}, \\
& 0 \leq \int_{\partial B_{r}\left(x_{0}\right)} \mathbb{1} \frac{}{B_{r}^{\varepsilon}\left(x_{0}\right)}\left|x_{m+1}\right|^{-\beta} v \cdot \nabla\left|v^{*}\right|^{2} \mathrm{~d} S(x) \\
& -\int_{B_{\sqrt{r^{2}-\varepsilon^{2}}}^{m}\left(x_{0}\right)} \varepsilon^{-\beta} e_{m+1} \cdot\left(\nabla\left|v^{*}\right|^{2}\left(x^{\prime}, \varepsilon\right)-\nabla\left|v^{*}\right|^{2}\left(x^{\prime},-\varepsilon\right)\right) \mathrm{d} x^{\prime}
\end{aligned}
$$

and, when $\beta \in(0,1)$,

$$
0 \leq \int_{\partial B_{r}\left(x_{0}\right)} \mathbb{1}_{\overline{B_{r}^{\varepsilon}\left(x_{0}\right)}}\left|x_{m+1}\right|^{\beta} v \cdot \nabla\left|\partial_{m+1} v\right|^{2} \mathrm{~d} S(x)
$$




$$
-\int_{B_{\sqrt[r^{2}-\varepsilon^{2}]{m}}^{m}\left(x_{0}\right)} \varepsilon^{\beta} e_{m+1} \cdot\left(\nabla\left|\partial_{m+1} v\right|^{2}\left(x^{\prime}, \varepsilon\right)-\nabla\left|\partial_{m+1} v\right|^{2}\left(x^{\prime},-\varepsilon\right)\right) \mathrm{d} x^{\prime}
$$

where $\mathbb{1} \frac{1}{B_{r}^{\varepsilon}\left(x_{0}\right)}$ is the indicator function of $\overline{B_{r}^{\varepsilon}\left(x_{0}\right)}, v$ is the outward unit normal on $\partial B_{r}\left(x_{0}\right)$, $\mathrm{d} S$ is the Lebesgue measure on $\partial B_{r}\left(x_{0}\right)$ and $B_{s}^{m}\left(x_{0}\right)=\left\{x \in \mathbb{R}^{m}:\left|x-x_{0}\right|<s\right\}$.

We consider the terms on the right hand side of (2.23)-(2.25) separately with a view to taking the limit as $\varepsilon \rightarrow 0^{+}$. Lemma 2.12 and Corollary 2.13 imply that $\partial_{i} v, \partial_{j} \partial_{i} v \in$ $W_{\beta}^{1,2}\left(B_{r}\left(x_{0}\right) ; \mathbb{R}^{n}\right)$ and are locally Hölder continuous in $B_{R}\left(x_{0}\right)$ for $i, j=1, \ldots, m$. The lemma and corollary further imply that $v^{*},\left(\partial_{i} v\right)^{*} \in W_{-\beta}^{1,2}\left(B_{r}\left(x_{0}\right) ; \mathbb{R}^{n}\right)$, where $i=1, \ldots, m$, are locally Hölder continuous in $B_{R}\left(x_{0}\right)$ and hence uniformly continuous in $\overline{B_{r}\left(x_{0}\right)}$. We can therefore check that $\left|\partial_{i} v\right|^{2} \in W_{\beta}^{1,2}\left(B_{r}\left(x_{0}\right)\right)$, integrating over $B_{r}^{\varepsilon}\left(x_{0}\right)$ and letting $\varepsilon \rightarrow 0^{+}$for the $m+1$ th derivative. It follows that

$$
\begin{aligned}
\left.|| x_{m+1}\right|^{\beta} v \cdot \nabla\left|\partial_{i} v\right|^{2} \mid & \leq 2\left|\left\langle v_{m+1}\left(\partial_{i} v\right)^{*}, \partial_{i} v\right\rangle\right|+\left.2 \sum_{j=1}^{m}|| x_{m+1}\right|^{\beta}\left\langle v_{j} \partial_{j} \partial_{i} v, \partial_{i} v\right\rangle \mid \\
& \leq C\left(1+\left|x_{m+1}\right|^{\beta}\right),
\end{aligned}
$$

where $C$ is a positive constant that may depend on $r$ but is independent of $\varepsilon$. Furthermore, since $\partial_{i} v$ and $\left(\partial_{i} v\right)^{*}$ are uniformly continuous in $\overline{B_{r}\left(x_{0}\right)}$, we see that

$$
\mathbb{1}_{B_{\sqrt{r^{2}-\varepsilon^{2}}}^{m}}\left(x_{0}\right) e_{m+1} \varepsilon^{\beta} \cdot\left(\nabla\left|\partial_{i} v\right|^{2}\left(x^{\prime}, \varepsilon\right)-\nabla\left|\partial_{i} v\right|^{2}\left(x^{\prime},-\varepsilon\right)\right) \rightarrow 0 \text { uniformly as } \varepsilon \rightarrow 0^{+}
$$

for $\left(x^{\prime}, 0\right) \in \overline{B_{r}\left(x_{0}\right)}$.

Since each $\partial_{j} \partial_{i} v \in W_{\beta}^{1,2}\left(B_{r}\left(x_{0}\right) ; \mathbb{R}^{n}\right)$ is locally Hölder continuous in $B_{R}\left(x_{0}\right)$ for $i, j=1, \ldots, m$ and $\operatorname{div}\left(\left|x_{m+1}\right|^{\beta} \nabla v\right)=0$ classically in $B_{R}\left(x_{0}\right) \backslash \partial \mathbb{R}_{+}^{m+1}$, we have $\left|x_{m+1}\right|^{-\beta} \partial_{m+1} v^{*}=-\Delta^{\prime} v \in W_{\beta}^{1,2}\left(B_{r}\left(x_{0}\right) ; \mathbb{R}^{n}\right)$ is uniformly continuous in $\overline{B_{r}\left(x_{0}\right)}$ for every $r<R$. We can hence check $\left|v^{*}\right|^{2} \in W_{-\beta}^{1,2}\left(B_{r}\left(x_{0}\right)\right)$. We also have

$$
\begin{aligned}
\left.|| x_{m+1}\right|^{-\beta} v \cdot \nabla\left|v^{*}\right|^{2} \mid & \leq\left. 2|| x_{m+1}\right|^{-\beta}\left\langle v_{m+1} \partial_{m+1} v^{*}, v^{*}\right\rangle\left|+2 \sum_{i=1}^{m}\right|\left|x_{m+1}\right|^{-\beta}\left\langle v_{i} \partial_{i} v^{*}, v^{*}\right\rangle \mid \\
& \leq C\left(1+\left|x_{m+1}\right|^{-\beta}\right)
\end{aligned}
$$

and

$\mathbb{1}_{B_{\sqrt{r^{2}-\varepsilon^{2}}}^{m}}\left(x_{0}\right) e_{m+1} \varepsilon^{-\beta} \cdot\left(\nabla\left|v^{*}\right|^{2}\left(x^{\prime}, \varepsilon\right)-\nabla\left|v^{*}\right|^{2}\left(x^{\prime},-\varepsilon\right)\right) \rightarrow 0$ uniformly as $\varepsilon \rightarrow 0^{+}$

for $\left(x^{\prime}, 0\right) \in \overline{B_{r}\left(x_{0}\right)}$.

In order to derive similar conclusions to (2.26) and (2.27) for the constituent integrands of (2.25), we assume that $\beta \in(0,1)$ and that $v$ is symmetric with respect to $\partial \mathbb{R}_{+}^{m+1}$, namely $v\left(x^{\prime}, x_{m+1}\right)=v\left(x^{\prime},-x_{m+1}\right)$ for every $\left(x^{\prime}, x_{m+1}\right) \in B_{R}\left(x_{0}\right)$. The symmetry of $v$ implies $v^{*}$ must be odd with respect to $\partial \mathbb{R}_{+}^{m+1}$, that is $v^{*}\left(x^{\prime}, x_{m+1}\right)=-v^{*}\left(x^{\prime},-x_{m+1}\right)$ for every $\left(x^{\prime}, x_{m+1}\right) \in B_{R}\left(x_{0}\right)$ and hence, as it is also continuous in $B_{R}\left(x_{0}\right)$ we have $v^{*}\left(x^{\prime}, 0\right)=0$ for every $\left(x^{\prime}, 0\right) \in B_{R}\left(x_{0}\right)$.

Fix $\left(x^{\prime}, 0\right) \in B_{R}\left(x_{0}\right)$ and note that $\left(x^{\prime}, 0\right) \in B_{r}\left(x_{0}\right)$ for some $r<R$ and choose $h$ with $|h|$ sufficiently small as to ensure $\left(x^{\prime}, h\right) \in B_{r}\left(x_{0}\right)$. We see that

$$
|h|^{-1}\left|v\left(x^{\prime}, h\right)-v\left(x^{\prime}, 0\right)\right|=\left|\partial_{m+1} v\left(x^{\prime}, x_{m+1}\right)\right|
$$




$$
\begin{aligned}
& =\left|x_{m+1}\right|^{-\beta}\left|v^{*}\left(x^{\prime}, x_{m+1}\right)-0\right| \\
& =\left|x_{m+1}\right|^{-\beta}\left|\partial_{m+1} v^{*}\left(x^{\prime}, \xi\right)\right|\left|x_{m+1}\right| \\
& \leq\left.\left|x_{m+1}\right||| \xi\right|^{-\beta} \partial_{m+1} v^{*}\left(x^{\prime}, \xi\right) \mid \\
& \leq C|h| \rightarrow 0 \text { as } h \rightarrow 0,
\end{aligned}
$$

where $x_{m+1}$ with $\left|x_{m+1}\right| \in(0,|h|)$ and $\xi$ with $|\xi| \in\left(0,\left|x_{m+1}\right|\right)$ are chosen such that the Mean Value Theorem holds. Thus we see that $\partial_{m+1} v\left(x^{\prime}, 0\right)=0$ classically for $\left(x^{\prime}, 0\right) \in B_{R}\left(x_{0}\right)$. Analogous calculations to those on the right hand side above show that $\partial_{m+1} v$ is continuous at $\left(x^{\prime}, 0\right)$ and hence continuous in $B_{R}\left(x_{0}\right)$. We also have

$$
\left|x_{m+1}\right|^{\beta} \partial_{m+1}^{2} v=\partial_{m+1} v^{*}-\beta x_{m+1}^{-1} v^{*}
$$

for $\left(x^{\prime}, x_{m+1}\right) \in \overline{B_{r}\left(x_{0}\right)} \backslash \partial \mathbb{R}_{+}^{m+1}$ and hence

$$
\begin{aligned}
\left.|| x_{m+1}\right|^{\beta} \partial_{m+1}^{2} v\left(x^{\prime}, x_{m+1}\right) \mid & \leq\left|\partial_{m+1} v^{*}\right|+\beta\left|x_{m+1}^{-1} v^{*}\right| \\
& \leq C+\beta\left|\partial_{m+1} v^{*}\left(x^{\prime}, \xi\right)\right| \\
& \leq C,
\end{aligned}
$$

in $\overline{B_{r}\left(x_{0}\right)} \backslash \partial \mathbb{R}_{+}^{m+1}$, where $\xi$ is chosen with $|\xi| \in\left(0,\left|x_{m+1}\right|\right)$ such that the Mean Value Theorem holds. It follows that $\left|x_{m+1}\right|^{\beta} \partial_{m+1}^{2} v$ is essentially bounded in $\overline{B_{r}\left(x_{0}\right)}$ and $\partial B_{r}\left(x_{0}\right)$.

The preceding discussion implies $\left|\partial_{m+1} v\right|^{2} \in W_{\beta}^{1,2}\left(B_{r}\left(x_{0}\right)\right)$ and

$$
\left.|| x_{m+1}\right|^{\beta} v \cdot \nabla\left|\partial_{m+1} v\right|^{2} \mid \leq C
$$

on $\overline{B_{r}\left(x_{0}\right)} \backslash \partial \mathbb{R}_{+}^{m+1}$. Furthermore, using the symmetry of $v$, we see that $\mathbb{1}_{B_{\sqrt{r^{2}-\varepsilon^{2}}}}\left(x_{0}\right) e_{m+1} \varepsilon^{\beta} \cdot\left(\nabla\left|\partial_{m+1} v\right|^{2}\left(x^{\prime}, \varepsilon\right)-\nabla\left|\partial_{m+1} v\right|^{2}\left(x^{\prime},-\varepsilon\right)\right) \rightarrow 0$ uniformly as $\varepsilon \rightarrow 0^{+}$

for $\left(x^{\prime}, 0\right) \in \overline{B_{r}\left(x_{0}\right)}$.

Using Lebesgue's Dominated Convergence Theorem, we combine (2.23) with (2.26) and (2.27), (2.24) with (2.28) and (2.29) and (2.25) with (2.30) and (2.31) to see that

$$
0 \leq \int_{\partial B_{r}\left(x_{0}\right)}\left|x_{m+1}\right|^{\beta} v \cdot \nabla\left|\partial_{i} v\right|^{2} \mathrm{~d} S(x), \quad 0 \leq \int_{\partial B_{r}\left(x_{0}\right)}\left|x_{m+1}\right|^{-\beta} v \cdot \nabla\left|v^{*}\right|^{2} \mathrm{~d} S(x)
$$

and, when $\beta \in(0,1)$ and $v$ is symmetric with respect to $\partial \mathbb{R}_{+}^{m+1}$,

$$
0 \leq \int_{\partial B_{r}\left(x_{0}\right)}\left|x_{m+1}\right|^{\beta} v \cdot \nabla\left|\partial_{m+1} v\right|^{2} \mathrm{~d} S(x)
$$

respectively. Noting that $\left|x_{m+1}\right|^{-\beta}\left|v^{*}\right|^{2}=\left|x_{m+1}\right|^{\beta}\left|\partial_{m+1} v\right|^{2}$, we apply Lemma 2.14 , to see that

$$
\begin{gathered}
s^{-(1+m+\beta)} \int_{B_{s}\left(x_{0}\right)}\left|x_{m+1}\right|^{\beta}\left|\nabla^{\prime} v\right|^{2} \mathrm{~d} x \leq r^{-(1+m+\beta)} \int_{B_{r}\left(x_{0}\right)}\left|x_{m+1}\right|^{\beta}\left|\nabla^{\prime} v\right|^{2} \mathrm{~d} x, \\
s^{-(1+m-\beta)} \int_{B_{s}\left(x_{0}\right)}\left|x_{m+1}\right|^{\beta}\left|\partial_{m+1} v\right|^{2} \mathrm{~d} x \leq r^{-(1+m-\beta)} \int_{B_{r}\left(x_{0}\right)}\left|x_{m+1}\right|^{\beta}\left|\partial_{m+1} v\right|^{2} \mathrm{~d} x
\end{gathered}
$$

and, when $\beta \in(0,1)$ and $v$ is symmetric with respect to $\partial \mathbb{R}_{+}^{m+1}$,

$$
s^{-(1+m+\beta)} \int_{B_{s}\left(x_{0}\right)}\left|x_{m+1}\right|^{\beta}\left|\partial_{m+1} v\right|^{2} \mathrm{~d} x \leq r^{-(1+m+\beta)} \int_{B_{r}\left(x_{0}\right)}\left|x_{m+1}\right|^{\beta}\left|\partial_{m+1} v\right|^{2} \mathrm{~d} x
$$


for every $0<s \leq r<R$. We apply Lebesgue's Dominated Convergence Theorem, sending $r \rightarrow R^{-}$and combine (2.32), (2.33) and (2.34) to see the Lemma holds for $0<s \leq r \leq R$.

Remark 2.15 We observe from (2.33) and (2.34) that without the symmetry condition on $v$, the monotonicity of the average energy of $\left|\partial_{m+1} v\right|^{2}$ is better than required if $\beta \in(-1,0]$ and may be worse than required if $\beta \in(0,1)$; the symmetry of $v$ resolves this issue by implying continuity of $\partial_{m+1} v$ on $\partial \mathbb{R}_{+}^{m+1}$ which, as the function given in Remark 2.9 illustrates, cannot be expected in general.

\subsection{Interior monotonicity formula}

We need a counterpart to Lemma 2.8 for balls in the interior of $\mathbb{R}_{+}^{m+1}$. Since the weight $x_{m+1}^{\beta}$ is not scale invariant on a (Euclidean) ball $B_{\rho}(y)$ with $y_{m+1} \geq 2 \rho$, we only expect monotonicity of the average energy (with respect to the Lebesgue measure) of solutions to $\operatorname{div}\left(x_{m+1}^{\beta} \nabla v\right)=$ 0 up to a correcting factor. As the radius tends to zero, the coefficients of uniform ellipticity for the preceding equation tend to constants. Accordingly, the correcting factor becomes smaller with the radius. General monotonicity-type formulas for linear uniformly elliptic equations are available in [14] for example. We perform the following calculations in order to determine how the correcting factor behaves explicitly as the radius decays geometrically. We first establish monotonicity on the boundary of concentric balls.

Lemma 2.16 Let $\beta \in(-1,1), B_{R}(y) \subset \mathbb{R}_{+}^{m+1}$ with $\overline{B_{R}(y)} \subset \mathbb{R}_{+}^{m+1}$ and suppose $v \in$ $C^{2}\left(B_{R}(y) ; \mathbb{R}^{n}\right)$ satisfies div $\left(x_{m+1}^{\beta} \nabla|v|^{2}\right) \geq 0$ classically in $B_{R}(y)$. Then, letting $\mathrm{d} S$ denote Lebesgue surface measure and sgn denote the sign function, for $0<s \leq r<R$ we have

$$
\begin{aligned}
& s^{-m} \frac{1}{\left(y_{m+1}-\operatorname{sgn}(\beta) s\right)^{\beta}} \int_{\partial B_{s}(y)} x_{m+1}^{\beta}|v|^{2} \mathrm{~d} S(x) \\
& \quad \leq r^{-m} \frac{1}{\left(y_{m+1}-\operatorname{sgn}(\beta) r\right)^{\beta}} \int_{\partial B_{r}(y)} x_{m+1}^{\beta}|v|^{2} \mathrm{~d} S(x) .
\end{aligned}
$$

Proof Let $\rho<R$. We calculate $0 \leq \int_{\partial B_{\rho}(y)} x_{m+1}^{\beta} v \cdot \nabla|v|^{2} \mathrm{~d} S(x)$, where $v$ is the unit outward normal on $\partial B_{\rho}(y)$. Now using variables $\rho=|x-y|$ and $\omega=\frac{x-y}{\rho}$ we have

$$
\begin{aligned}
0 \leq & \rho^{m} \int_{\mathbb{S}^{m}}\left(\rho \omega_{m+1}+y_{m+1}\right)^{\beta} \frac{\partial}{\partial \rho}\left(|v(\rho \omega+y)|^{2}\right) \mathrm{d} \omega \\
= & \rho^{m} \int_{\mathbb{S}^{m}} \frac{\partial}{\partial \rho}\left(\left(\rho \omega_{m+1}+y_{m+1}\right)^{\beta}|v(\rho \omega+y)|^{2}\right) \mathrm{d} \omega \\
& -\rho^{m} \int_{\mathbb{S}^{m}} \beta \omega_{m+1}\left(\rho \omega_{m+1}+y_{m+1}\right)^{\beta-1}|v(\rho \omega+y)|^{2} \mathrm{~d} \omega \\
= & \rho^{m} \frac{\partial}{\partial \rho}\left(\rho^{-m} \int_{\partial B_{\rho}(y)} x_{m+1}^{\beta}|v|^{2} \mathrm{~d} S(x)\right)-\int_{\partial B_{\rho}(y)} \frac{\beta}{\rho} x_{m+1}^{\beta-1}\left(x_{m+1}-y_{m+1}\right)|v|^{2} \mathrm{~d} S(x) .
\end{aligned}
$$

We define $f(\rho)=\rho^{-m} \int_{\partial B_{\rho}(y)} x_{m+1}^{\beta}|v|^{2} \mathrm{~d} S(x)$ and divide by $\rho^{m}$ to see that

$$
\begin{aligned}
0 & \leq f^{\prime}(\rho)-\frac{\beta}{\rho} f(\rho)+\frac{y_{m+1} \beta}{\rho^{m+1}} \int_{\partial B_{\rho}(y)} x_{m+1}^{\beta-1}|v|^{2} \mathrm{~d} S(x) \\
& \leq f^{\prime}(\rho)-\frac{\beta}{\rho} f(\rho)+\frac{y_{m+1} \beta}{\rho\left(y_{m+1}-\operatorname{sgn}(\beta) \rho\right)} f(\rho)
\end{aligned}
$$




$$
=f^{\prime}(\rho)+\left(\frac{|\beta|}{y_{m+1}-\operatorname{sgn}(\beta) \rho}\right) f(\rho) .
$$

Hence $0 \leq\left(\left(y_{m+1}-\operatorname{sgn}(\beta) \rho\right)^{-\beta} f(\rho)\right)^{\prime}$ and integrating between $s<r$ concludes the proof.

With this lemma in hand we can establish the following counterpart to Lemma 2.8.

Lemma 2.17 Let $B_{R}(y) \subset \mathbb{R}^{m+1}$ with $y_{m+1} \geq \theta R$ for $\theta \geq 2$. Suppose $v \in C^{2}\left(B_{R}(y) ; \mathbb{R}^{n}\right) \cap$ $W_{\beta}^{1,2}\left(B_{R}(y) ; \mathbb{R}^{n}\right)$ satisfies $\operatorname{div}\left(x_{m+1}^{\beta} \nabla v\right)=0$ in $B_{R}(y)$. Then there exists $C=C(m)$ such that

$$
\left(\frac{R}{2}\right)^{-(m+1)} \int_{B_{\frac{R}{2}}(y)} x_{m+1}^{\beta}|\nabla v|^{2} \mathrm{~d} x \leq\left(1+\frac{C}{\theta-1}\right) R^{-(m+1)} \int_{B_{R}(y)} x_{m+1}^{\beta}|\nabla v|^{2} \mathrm{~d} x .
$$

Proof Let $g(r):=\int_{B_{r}(y)} x_{m+1}^{\beta}|f|^{2} \mathrm{~d} x$ where $f$ satisfies the assumptions of Lemma 2.16 and $r \in(0, R)$. Then we have

$$
\begin{aligned}
g(r) & =\int_{0}^{r} \int_{\partial B_{\rho}(y)} x_{m+1}^{\beta}|f|^{2} \mathrm{~d} S(x) \mathrm{d} \rho \\
& =\int_{0}^{r}\left(y_{m+1}-\operatorname{sgn}(\beta) \rho\right)^{\beta} \rho^{m}\left(y_{m+1}-\operatorname{sgn}(\beta) \rho\right)^{-\beta} \rho^{-m} \int_{\partial B_{\rho}(y)} x_{m+1}^{\beta}|f|^{2} \mathrm{~d} S(x) \mathrm{d} \rho \\
& \leq \int_{0}^{r}\left(y_{m+1}-\operatorname{sgn}(\beta) \rho\right)^{\beta} \rho^{m} \mathrm{~d} \rho\left(y_{m+1}-\operatorname{sgn}(\beta) r\right)^{-\beta} r^{-m} \int_{\partial B_{r}(y)} x_{m+1}^{\beta}|f|^{2} \mathrm{~d} S(x) \\
& \leq y_{m+1}^{\beta} \frac{r}{m+1}\left(y_{m+1}-\operatorname{sgn}(\beta) r\right)^{-\beta} \int_{\partial B_{r}(y)} x_{m+1}^{\beta}|f|^{2} \mathrm{~d} S(x) .
\end{aligned}
$$

Hence

$$
\begin{aligned}
0 & \leq g^{\prime}(r)-g(r) y_{m+1}^{-\beta} \frac{m+1}{r}\left(y_{m+1}-\operatorname{sgn}(\beta) r\right)^{\beta} \\
& =g^{\prime}(r)-g(r) \frac{m+1}{r}\left(1-\operatorname{sgn}(\beta) \frac{r}{y_{m+1}}\right)^{\beta} \\
& =g^{\prime}(r)-g(r) \frac{m+1}{r}\left(1+\beta\left(1-\operatorname{sgn}(\beta) \frac{s}{y_{m+1}}\right)^{\beta-1} \operatorname{sgn}(\beta) \frac{-r}{y_{m+1}}\right) \\
& =g^{\prime}(r)-g(r) \frac{m+1}{r}+g(r) \frac{m+1}{r}|\beta|\left(1-\operatorname{sgn}(\beta) \frac{s}{y_{m+1}}\right)^{\beta-1} \frac{r}{y_{m+1}},
\end{aligned}
$$

where $s \in(0, r)$ is such that the Mean Value Theorem holds for the function $r \mapsto(1-$ $\left.\operatorname{sgn}(\beta) \frac{r}{y_{m+1}}\right)^{\beta}$. Now recall that $y_{m+1} \geq \theta R \geq \theta r$ for $\theta \geq 2$. We hence find

$$
0 \leq g^{\prime}(r)-g(r) \frac{m+1}{r}+g(r) \frac{m+1}{r}|\beta| \frac{1}{\theta-1} .
$$

It follows that $0 \leq\left(r^{-(m+1)} r \frac{|\beta|(m+1)}{\theta-1} g(r)\right)^{\prime}$ and consequently, if $\frac{R}{2} \leq r<R$, we have

$$
\begin{aligned}
\left(\frac{R}{2}\right)^{-(m+1)} g\left(\frac{R}{2}\right) & \leq 2^{\frac{|\beta|(m+1)}{\theta-1}} r^{-(m+1)} g(r) \\
& =\left(1+\frac{|\beta|(m+1)}{\theta-1} \xi^{\frac{|\beta|(m+1)}{\theta-1}-1}\right) r^{-(m+1)} g(r)
\end{aligned}
$$




$$
\leq\left(1+\frac{|\beta|(m+1)}{\theta-1} 2^{|\beta|(m+1)}\right) r^{-(m+1)} g(r)
$$

where $\xi \in(1,2)$ is such that the Mean Value theorem holds for the function $t \mapsto$ $t^{\frac{|\beta|(m+1)}{\theta-1}}$. Since $\operatorname{div}\left(x_{m+1}^{\beta} \nabla v\right)=0$, as observed in the proof of Lemma 2.8, we have $\operatorname{div}\left(x_{m+1}^{\beta} \nabla\left|\partial_{i} v\right|^{2}\right) \geq 0$ for $i=1, \ldots, m$ and $\operatorname{div}\left(x_{m+1}^{-\beta} \nabla\left|x_{m+1}^{\beta} \partial_{m+1} v\right|^{2}\right) \geq 0$. We apply (2.36) with $f=\partial_{i} v$ for $i=1, \ldots, m$, and with $f=x_{m+1}^{\beta} \partial_{m+1} v$ and $-\beta$ in place of $\beta$ and combine the results, letting $r \rightarrow R^{-}$to conclude the proof.

\subsection{Solutions of the linear degenerate Dirichlet problem}

We require further results regarding the following Dirichlet problem: solve

$$
\operatorname{div}\left(\left|x_{m+1}\right|^{\beta} \nabla v\right)=0 \text { in } B_{R}\left(x_{0}\right) \quad \text { and } \quad v=\phi \text { on } \partial B_{R}\left(x_{0}\right)
$$

for a given $\phi$, where $x_{0} \in \mathbb{R}^{m+1}$, in order to apply a version of the method of harmonic replacement in the proof of Lemma 4.23. A weak solution of (2.37) is a $v \in W_{\beta}^{1,2}\left(B_{R}\left(x_{0}\right) ; \mathbb{R}^{n}\right)$ which weakly satisfies $\operatorname{div}\left(\left|x_{m+1}\right|^{\beta} \nabla v\right)=0$ in $B_{R}\left(x_{0}\right)$ with $v-\phi \in W_{\beta, 0}^{1,2}\left(B_{R}\left(x_{0}\right) ; \mathbb{R}^{n}\right)$. We collect the results we require, which can be found in [19], in the form of a lemma.

Lemma 2.18 Suppose $\phi \in W_{\beta}^{1,2}\left(B_{R}\left(x_{0}\right) ; \mathbb{R}^{n}\right)$. Then there exists a $v \in W_{\beta}^{1,2}\left(B_{R}\left(x_{0}\right) ; \mathbb{R}^{n}\right)$ which is a weak solution of the Dirichlet problem (2.37). Any such solution is unique and continuous in $B_{R}\left(x_{0}\right)$, if $\phi \in C\left(\overline{B_{R}\left(x_{0}\right)} ; \mathbb{R}^{n}\right)$ then $v(x) \rightarrow \phi(z)$ as $x \rightarrow z$ for $z \in \partial B_{R}\left(x_{0}\right)$ and the weak maximum principle

$$
\frac{\max }{B_{R}\left(x_{0}\right)} v=\max _{\partial B_{R}\left(x_{0}\right)} v=\max _{\partial B_{R}\left(x_{0}\right)} \phi
$$

and weak minimum principle

$$
\frac{\min }{B_{R}\left(x_{0}\right)} v=\min _{\partial B_{R}\left(x_{0}\right)} v=\min _{\partial B_{R}\left(x_{0}\right)} \phi
$$

both hold, where we take the maximum and minimum component-wise. If $w \in W_{\beta}^{1,2}\left(B_{R}\left(x_{0}\right)\right.$; $\left.\mathbb{R}^{n}\right)$ also satisfies $w-\phi \in W_{\beta, 0}^{1,2}\left(B_{R}\left(x_{0}\right) ; \mathbb{R}^{n}\right)$ then

$$
\int_{B_{R}\left(x_{0}\right)}\left|x_{m+1}\right|^{\beta}|\nabla v|^{2} \mathrm{~d} x \leq \int_{B_{R}\left(x_{0}\right)}\left|x_{m+1}\right|^{\beta}|\nabla w|^{2} \mathrm{~d} x .
$$

Proof Since $\left|x_{m+1}\right|^{\beta}$ is of Muckenhoupt class $A_{2}$ it follows from 1.6 of [19] that $\left|x_{m+1}\right|^{\beta}$ is a 2-admissible weight so we may apply the theory of [19]. Aside from the minimising property of $v$, the assertions of the lemma are consequences of Theorem 3.70, Corollary 6.32, the strong maximum principle 6.5 and lastly 3.17 in [19]. If $v$ is a weak solution of (2.37) for a given $\phi$ and $w \in W_{\beta}^{1,2}\left(B_{R}\left(x_{0}\right) ; \mathbb{R}^{n}\right)$ with $w-\phi \in W_{\beta, 0}^{1,2}\left(B_{R}\left(x_{0}\right) ; \mathbb{R}^{n}\right)$ then $w-v \in W_{\beta, 0}^{1,2}\left(B_{R}\left(x_{0}\right) ; \mathbb{R}^{n}\right)$. Hence, by approximation, we have

$$
\int_{B_{R}\left(x_{0}\right)}\left|x_{m+1}\right|^{\beta}\langle\nabla v, \nabla(w-v)\rangle \mathrm{d} x=0,
$$

so that

$$
\int_{B_{R}\left(x_{0}\right)}\left|x_{m+1}\right|^{\beta}|\nabla w|^{2} \mathrm{~d} x=\int_{B_{R}\left(x_{0}\right)}\left|x_{m+1}\right|^{\beta}|\nabla v|^{2} \mathrm{~d} x+\int_{B_{R}\left(x_{0}\right)}\left|x_{m+1}\right|^{\beta}|\nabla(w-v)|^{2} \mathrm{~d} x
$$

which concludes the proof. 
The uniqueness of solutions to the Dirichlet problem (2.37) implies that solutions with boundary data which are symmetric with respect to $\partial \mathbb{R}_{+}^{m+1}$ are themselves symmetric. More precisely, we have the following.

Lemma 2.19 Let $x_{0} \in \partial \mathbb{R}_{+}^{m+1}$ and suppose $v, \phi \in W_{\beta}^{1,2}\left(B_{R}\left(x_{0}\right) ; \mathbb{R}^{n}\right)$ and $v$ is a weak solution of the Dirichlet problem (2.37) with $\phi$ as boundary data. Let $\phi \in C\left(\overline{B_{R}\left(x_{0}\right)} ; \mathbb{R}^{n}\right)$ and suppose $\phi\left(x^{\prime}, x_{m+1}\right)=\phi\left(x^{\prime},-x_{m+1}\right)$ for every $\left(x^{\prime}, x_{m+1}\right) \in \overline{B_{R}\left(x_{0}\right)}$. Then $v\left(x^{\prime}, x_{m+1}\right)=$ $v\left(x^{\prime},-x_{m+1}\right)$ for every $\left(x^{\prime}, x_{m+1}\right) \in B_{R}\left(x_{0}\right)$.

Proof The continuity of $\phi$ in $\overline{B_{R}\left(x_{0}\right)}$, combined with an application of Lemma 2.18, implies that $v$ and, consequently, $\tilde{v}\left(x^{\prime}, x_{m+1}\right):=v\left(x^{\prime},-x_{m+1}\right)$ are continuous in $\overline{B_{R}\left(x_{0}\right)}$. We observe that $\tilde{v} \in W_{\beta}^{1,2}\left(B_{R}\left(x_{0}\right) ; \mathbb{R}^{n}\right)$ weakly satisfies $\operatorname{div}\left(\left|x_{m+1}\right|^{\beta} \nabla \tilde{v}\right)=0$ in $B_{R}\left(x_{0}\right)$ and $\left.\tilde{v}\right|_{\partial B_{R}\left(x_{0}\right)}=$ $\left.\phi\right|_{\partial B_{R}\left(x_{0}\right)}$ so that $\tilde{v}-\phi \in W_{\beta, 0}^{1,2}\left(B_{R}\left(x_{0}\right) ; \mathbb{R}^{n}\right)$. Hence $v$ and $\tilde{v}$ solve the same Dirichlet problem; solutions to this problem are unique by Lemma 2.18 and thus $\tilde{v}=v$.

\section{Intrinsic fractional harmonic maps}

We assume, translating $N$ if necessary, that $0 \in N$. For reasons discussed in the introduction we always assume $m \geq 2$; when $m \geq 3$ we let $\beta \in(-1,1)$ and when $m=2$ we let $\beta \in\left(-3^{-1}, 1\right)$. This allows us to apply Lemma 2.3 and we can define

$$
\dot{W}_{\beta}^{1,2}\left(\mathbb{R}_{+}^{m+1} ; N\right)=\left\{v \in \dot{W}_{\beta}^{1,2}\left(\mathbb{R}_{+}^{m+1} ; \mathbb{R}^{n}\right): v(x) \in N \text { for almost every } x \in \mathbb{R}_{+}^{m+1}\right\} .
$$

Henceforth, in this section and Sect. 4, we fix an open $\mathcal{O} \subset \partial \mathbb{R}_{+}^{m+1}$ such that a continuous linear trace operator with respect to $\mathcal{O}$ exists; we do not assume $\mathcal{O}$ is necessarily the whole of $\partial \mathbb{R}_{+}^{m+1}$. We can, for example, obtain such a trace operator $T: \dot{W}_{\beta}^{1,2}\left(\mathbb{R}_{+}^{m+1} ; \mathbb{R}^{n}\right) \rightarrow$ $L^{p}\left(\mathcal{O} ; \mathbb{R}^{n}\right)$, where $p=p(\beta) \in(1,2]$, by combining Lemmata 2.1 and 2.3 with [12, Section 4.3, Theorem 1] whenever $\mathcal{O}$ is contained in the boundary of a Lipschitz $\Omega \subset \mathbb{R}_{+}^{m+1}$. Define

$$
I^{\beta}(u)=\inf \left\{E^{\beta}(v): v \in \dot{W}_{\beta}^{1,2}\left(\mathbb{R}_{+}^{m+1} ; N\right), T v=u\right\}
$$

for $u \in T\left(\dot{W}_{\beta}^{1,2}\left(\mathbb{R}_{+}^{m+1} ; N\right)\right)$. Recall from the introduction that $I^{\beta}$ serves as an intrinsic energy for $u$; it does not depend on the choice of embedding of $N$ into Euclidean space. Moreover, $I^{\beta}$ coincides with the square of the fractional Sobolev norm $\|u\|_{\dot{H}^{\frac{1-\beta}{2}}}\left(\partial \mathbb{R}_{+}^{m+1} ; \mathbb{R}^{n}\right)$ when $N=\mathbb{R}^{n}$ and $\mathcal{O}=\partial \mathbb{R}_{+}^{m+1}$.

For every $u \in T\left(\dot{W}_{\beta}^{1,2}\left(\mathbb{R}_{+}^{m+1} ; N\right)\right)$, an application of the direct method of the calculus of variations shows that there exists $v \in \dot{W}_{\beta}^{1,2}\left(\mathbb{R}_{+}^{m+1} ; N\right)$ with $T v=u$, where $T$ is the trace operator with respect to $\mathcal{O}$, such that $I^{\beta}(u)=E^{\beta}(v)$. For a given $u$, such a $v$ is referred to henceforth as a minimal harmonic map. Any minimal harmonic map $v$ is weakly harmonic in $\mathbb{R}_{+}^{m+1}$ with respect to the metric represented in Euclidean coordinates by $x_{m+1}^{\alpha} \delta_{i j}$, where $\beta=\frac{\alpha(m-1)}{2}$; the Dirichlet energy on $\mathbb{R}_{+}^{m+1}$ for this metric is precisely $E^{\beta}$. Such a $v$ therefore satisfies

$$
\int_{\mathbb{R}_{+}^{m+1}} x_{m+1}^{\beta}(\langle\psi, A(v)(\nabla v, \nabla v)\rangle-\langle\nabla v, \nabla \psi\rangle) \mathrm{d} x=0
$$


for every $\phi \in C_{0}^{\infty}\left(\mathbb{R}_{+}^{m+1} ; \mathbb{R}^{n}\right)$, where $A$ is the second fundamental form of $N$ and

$$
\langle\nabla v, \nabla \psi\rangle=\sum_{i=1}^{m+1}\left\langle\partial_{i} v, \partial_{i} \psi\right\rangle \quad \text { and } A(v)(\nabla v, \nabla v)=\sum_{i=1}^{m+1} A(v)\left(\partial_{i} v, \partial_{i} v\right) .
$$

Formally, if $v$ is sufficiently regular in $\mathbb{R}_{+}^{m+1} \cup \mathcal{O}$, we calculate

$\int_{\mathbb{R}_{+}^{m+1}} x_{m+1}^{\beta}(\langle\psi, A(v)(\nabla v, \nabla v)\rangle-\langle\nabla v, \nabla \psi\rangle) \mathrm{d} x=\int_{\mathcal{O}}\left\langle\left(x_{m+1}^{\beta} \partial_{m+1} v\right)\left(x^{\prime}, 0\right), \phi\left(x^{\prime}\right)\right\rangle \mathrm{d} x^{\prime}$,

for every $\phi \in C_{0}^{\infty}\left(\mathcal{O} ; \mathbb{R}^{n}\right)$ and any $\psi \in \mathcal{D}_{+}\left(\mathbb{R}_{+}^{m+1} ; \mathbb{R}^{n}\right)$ with $\psi\left(x^{\prime}, 0\right)=\phi\left(x^{\prime}\right)$, where $\mathrm{d} x^{\prime}$ is the Lebsegue measure on $\mathbb{R}^{m}$. In general, the integral in (3.1) defines a distribution on $\mathcal{O}$ given by

$$
\partial_{m+1}^{\beta} v(\phi):=\int_{\mathbb{R}_{+}^{m+1}} x_{m+1}^{\beta}(\langle\psi, A(v)(\nabla v, \nabla v)\rangle-\langle\nabla v, \nabla \psi\rangle) \mathrm{d} x
$$

for $\phi \in C_{0}^{\infty}\left(\mathcal{O} ; \mathbb{R}^{n}\right)$. This observation allows us, analogously to [30, Proposition 1.1], to identify a superdifferential for $I^{\beta}$. Recall that since $N$ is compact, Theorem 1 in Section 2.12.3 of [39] gives a tubular neighbourhood of $N$, which has the form $U_{\delta}(N)=\{x \in$ $\left.\mathbb{R}^{n}: \operatorname{dist}(x, N)<\delta\right\}$ for a $\delta=\delta(N)>0$, and a smooth map $\pi_{N}: U_{\delta}(N) \rightarrow N$ such that $\left|\pi_{N}(y)-y\right|=\operatorname{dist}(y, N)$ for every $y \in U_{\delta}(N)$. Using the same method of proof as [30, Proposition 1.1] we deduce the following.

Lemma 3.1 Let $u \in T\left(\dot{W}_{\beta}^{1,2}\left(\mathbb{R}_{+}^{m+1} ; N\right)\right)$ and $v \in \dot{W}_{\beta}^{1,2}\left(\mathbb{R}_{+}^{m+1} ; N\right)$ be a minimal harmonic map with $T v=u$. Then for $\phi \in C_{0}^{\infty}\left(\mathcal{O} ; \mathbb{R}^{n}\right)$,

$$
I^{\beta}\left(\pi_{N}(u+t \phi)\right) \leq I^{\beta}(u)-t \partial_{m+1}^{\beta} v(\phi)+o(|t|)
$$

as $t \rightarrow 0$.

It follows from this proposition that if $\left.\frac{\partial}{\partial t}\right|_{t=0} I^{\beta}\left(\pi_{N}(u+t \phi)\right)$ exists then it is equal to $-\partial_{m+1}^{\beta} v(\phi)$ where $v$ is any minimal harmonic map with $T v=u$; this indicates a candidate for the first variation of $I^{\beta}$.

Definition 3.2 Let $\beta \in(-1,1)$ and $\mathcal{D}_{\beta}$ be the collection of all $u \in T\left(\dot{W}_{\beta}^{1,2}\left(\mathbb{R}_{+}^{m+1} ; N\right)\right)$ such that there exists a distribution $\lambda_{\beta} \in\left(C_{0}^{\infty}\left(\mathcal{O} ; \mathbb{R}^{n}\right)\right)^{*}$ with $\lambda_{\beta}=-\partial_{m+1}^{\beta} v$ for every minimal harmonic map $v \in \dot{W}_{\beta}^{1,2}\left(\mathbb{R}_{+}^{m+1} ; N\right)$ with $T v=u$. Then we may define a map $\Lambda_{\beta}: \mathcal{D}_{\beta} \rightarrow\left(C_{0}^{\infty}\left(\mathcal{O} ; \mathbb{R}^{n}\right)\right)^{*}: u \mapsto \lambda_{\beta}=\Lambda_{\beta} u$.

In [30, Theorem 1.1] Moser showed that $\Lambda_{0}$ is the first variation of $I^{0}$. The method of proof of Moser's theorem, applied with the Lebesgue measure $\mathrm{d} x$ on $\mathbb{R}^{m+1}$ replaced by $x_{m+1}^{\beta} \mathrm{d} x$, yields the following.

Lemma 3.3 If $u \in \mathcal{D}_{\beta}$, then

$$
\left.\frac{\partial}{\partial t}\right|_{t=0} I^{\beta}\left(\pi_{N}(u+t \phi)\right)=\Lambda_{\beta} u(\phi)
$$

for all $\phi \in C_{0}^{\infty}\left(\mathcal{O} ; \mathbb{R}^{n}\right)$. If $u \notin \mathcal{D}_{\beta}$, then there exists $\phi \in C_{0}^{\infty}\left(\mathcal{O} ; \mathbb{R}^{n}\right)$ such that the function $t \mapsto I^{\beta}\left(\pi_{N}(u+t \phi)\right)$ is not differentiable at 0 . 
Consequently, we may define intrinsic fractional harmonic maps as follows.

Definition 3.4 Let $\beta \in(-1,1)$ and $u \in T\left(\dot{W}_{\beta}^{1,2}\left(\mathbb{R}_{+}^{m+1} ; N\right)\right)$. If $\Lambda_{\beta} u=0$ then we say that $u$ is an intrinsic $\frac{1-\beta}{2}$-harmonic map.

As discussed in the introduction, intrinsic $\frac{1-\beta}{2}$-harmonic maps are the boundary values of free boundary harmonic maps from $\mathbb{R}_{+}^{m+1}$ to $N$. Such maps may have singularities in $\mathcal{O}$ and we may not expect regularity in general. We consider a smaller class of fractional harmonic maps which locally minimise $I^{\beta}$ in order to obtain partial regularity in $\mathcal{O}$.

Definition 3.5 We say that $u \in T\left(\dot{W}_{\beta}^{1,2}\left(\mathbb{R}_{+}^{m+1} ; N\right)\right)$ is an intrinsic locally minimising $\frac{1-\beta}{2}$ harmonic map, or a local minimiser of $I^{\beta}$, if for every compact $K \subset \mathcal{O}$ and every $\tilde{u} \in$ $T\left(\dot{W}_{\beta}^{1,2}\left(\mathbb{R}_{+}^{m+1} ; N\right)\right)$ with $\left.u\right|_{\mathcal{O} \backslash K}=\left.\tilde{u}\right|_{\mathcal{O} \backslash K}$ we have $I^{\beta}(u) \leq I^{\beta}(\tilde{u})$.

This definition allows us to consider interior regularity for a class of critical points of $I^{\beta}$ without explicitly specifying boundary conditions. For example, minimisers of $I^{\beta}$ with respect to Dirichlet or free boundary conditions satisfy the definition. In order to deduce regularity results for $u$, we analyse their minimal harmonic extensions in more detail. To this end, we make the following definition.

Definition 3.6 Let $v \in \dot{W}_{\beta}^{1,2}\left(\mathbb{R}_{+}^{m+1} ; N\right)$. We say that $v$ is $E^{\beta}$ minimising, or energy minimising, in $\mathbb{R}_{+}^{m+1}$ relative to $\mathcal{O} \subset \partial \mathbb{R}_{+}^{m+1}$, if for every compact $K \subset \mathbb{R}^{m+1}$ with $K \cap \partial \mathbb{R}_{+}^{m+1} \subset \mathcal{O}$ and for every $w \in \dot{W}_{\beta}^{1,2}\left(\mathbb{R}_{+}^{m+1} ; N\right)$ with $\left.v\right|_{\mathbb{R}_{+}^{m+1} \backslash K}=\left.w\right|_{\mathbb{R}_{+}^{m+1} \backslash K}$ we have $E^{\beta}(v) \leq E^{\beta}(w)$.

Local minimisers of $I^{\beta}$ and minimisers of $E^{\beta}$ relative to $\mathcal{O}$ are connected as follows.

Lemma 3.7 Suppose $u \in T\left(\dot{W}_{\beta}^{1,2}\left(\mathbb{R}_{+}^{m+1} ; N\right)\right)$ locally minimises $I^{\beta}$ in the sense of Definition 4.11 and fix a minimal harmonic map $v \in \dot{W}_{\beta}^{1,2}\left(\mathbb{R}_{+}^{m+1} ; N\right)$ with $T v=u$. Then $v$ is a minimiser of $E^{\beta}$ relative to $\mathcal{O}$.

Proof Let $K \subset \mathbb{R}^{m+1}$ be compact such that the compact set $K_{m}:=K \cap \partial \mathbb{R}_{+}^{m+1} \subset \mathcal{O}$ and suppose that $w \in \dot{W}_{\beta}^{1,2}\left(\mathbb{R}_{+}^{m+1} ; N\right)$ satisfies $\left.v\right|_{\mathbb{R}_{+}^{m+1} \backslash K}=\left.w\right|_{\mathbb{R}_{+}^{m+1} \backslash K}$. Define $\tilde{u}=T w$ and let $\tilde{v}$ be a minimal harmonic map with $T \tilde{v}=\tilde{u}$. Since $\mathcal{O}$ is open in $\partial \mathbb{R}_{+}^{m+1}$ and $K_{m} \subset \mathcal{O}$ is compact we have $\operatorname{dist}^{m}\left(K_{m} ; \partial \mathcal{O}\right)>0$, where $\operatorname{dist}^{m}$ is the distance in $\partial \mathbb{R}_{+}^{m+1}$. We can therefore choose an open set $\tilde{\mathcal{O}} \subset \mathcal{O}$ with $K_{m} \subset \tilde{\mathcal{O}} \subset \overline{\mathcal{O}} \subset \mathcal{O}$. Since $K_{m}$ is closed and $\tilde{\mathcal{O}}$ is open we have $\operatorname{dist}^{m}\left(K_{m} ; \partial \tilde{\mathcal{O}}\right)>0$ as well. It follows that $\operatorname{dist}(\mathcal{O} \backslash \overline{\mathcal{O}} ; K):=\kappa>0$, where dist is the Euclidean distance in $\overline{\mathbb{R}_{+}^{m+1}}$. The continuity of the trace operator yields

$$
\int_{\mathcal{O} \backslash \tilde{\mathcal{O}}}|u-\tilde{u}|^{p} \mathrm{~d} x=\int_{\mathcal{O} \backslash \tilde{\mathcal{O}}}|T(v-w)|^{p} \mathrm{~d} x \leq C|| v-\left.w\right|_{W^{1, p}\left((\mathcal{O} \backslash \tilde{\mathcal{O}}) \times(0, \kappa) ; \mathbb{R}^{n}\right)} ^{p}=0,
$$

since $v=w$ in $\mathcal{O} \backslash \overline{\tilde{\mathcal{O}}} \times(0, \kappa)$, where $p$ is the number from Lemma 2.1 depending on $\beta$. Since $v$ and $\tilde{v}$ are minimal harmonic maps and $u$ is a minimiser of $I^{\beta}$, we have

$$
E^{\beta}(v)=I^{\beta}(u) \leq I^{\beta}(\tilde{u})=E^{\beta}(\tilde{v}) \leq E^{\beta}(w)
$$

as required. 
As a consequence of the preceding lemma, we can consider the regularity of minimisers of $E^{\beta}$ relative to $\mathcal{O}$ on relatively open balls (in the Euclidean topology) centred on $\mathbb{R}_{+}^{m+1} \cup \mathcal{O}$ in order to prove regularity of fractional harmonic maps. Our main results, stated and proved in Sect. 4 , constitute an $\varepsilon$-regularity and a corresponding partial regularity theorem for minimisers of $E^{\beta}$ relative to $\mathcal{O}$ which translate into the following partial regularity result for fractional harmonic maps.

Theorem 3.8 Fix $\mathcal{O} \subset \partial \mathbb{R}_{+}^{m+1}$. When $m \geq 3$ let $\beta \in(-1,1)$ and when $m=2$ let $\beta \in$ $\left(-3^{-1}, 1\right)$. Suppose $u \in T\left(\dot{W}_{\beta}^{1,2}\left(\mathbb{R}_{+}^{m+1} ; N\right)\right)$ is a local minimiser of $I^{\beta}$. Then there exists a relatively closed set $\Sigma \subset \mathcal{O}$ with $\mathcal{H}^{m+\beta-1}(\Sigma)=0$ such that $u \in C^{\infty}(\mathcal{O} \backslash \Sigma ; N)$.

Proof Fix a minimal harmonic map $v \in \dot{W}_{\beta}^{1,2}\left(\mathbb{R}_{+}^{m+1} ; N\right)$ with $T v=u$. As $u$ is a minimiser of $I^{\beta}$, Lemma 3.7 implies $v$ is a minimiser of $E^{\beta}$ relative to $\mathcal{O}$. An application of Theorem 4.3 implies the result.

\section{Partial regularity of minimisers of $E^{\beta}$ relative to $\mathcal{O}$}

The main result of this section is the following $\varepsilon$-regularity theorem for minimisers of $E^{\beta}$ relative to $\mathcal{O}$. To state the theorem and subsequent results we will need the following notation. For a set $\Omega \subset \mathbb{R}_{+}^{m+1}$ we will sometimes split the boundary $\partial \Omega$ into the (possibly empty) sets $\partial^{+} \Omega=\partial \Omega \cap \mathbb{R}_{+}^{m+1}$ and $\partial^{0} \Omega=\partial \Omega \cap \partial \mathbb{R}_{+}^{m+1}$. Let $x_{0} \in \partial \mathbb{R}_{+}^{m+1}$ and recall the notation $B_{R}^{+}\left(x_{0}\right)=\left\{x \in \mathbb{R}_{+}^{m+1}:\left|x-x_{0}\right|<R\right\}$.

Theorem 4.1 If $m \geq 3$, let $\beta \in(-1,1)$ and if $m=2$ let $\beta \in\left(-3^{-1}, 1\right)$. Let $v \in \dot{W}_{\beta}^{1,2}\left(\mathbb{R}_{+}^{m+1} ; N\right)$ be a minimiser of $E^{\beta}$ relative to $\mathcal{O}$, let $x_{0} \in \partial \mathbb{R}_{+}^{m+1}$ and $B_{R}^{+}\left(x_{0}\right)$ be a half-ball with $R \leq 1$ and $\overline{\partial^{0} B_{R}^{+}\left(x_{0}\right)} \subset \mathcal{O}$. There exists $\varepsilon=\varepsilon(m, N, \beta)>0$ such that the following holds. If $R^{1-m-\beta} \int_{B_{R}^{+}\left(x_{0}\right)} x_{m+1}^{\beta}|\nabla v|^{2} \mathrm{~d} x \leq \varepsilon$ then there is a $\theta=\theta(m, N, \beta) \in(0,1)$ and $a \gamma=\gamma(m, N, \beta) \in(0,1)$ such that $\left.v \in C^{0, \gamma} \overline{\left(B_{\theta R}^{+}\left(x_{0}\right)\right.} ; N\right)$. Furthermore, for every $l \in \mathbb{N}$ there is a $\theta=\theta(m, N, \beta, l) \in(0,1)$ and $a \gamma=\gamma(m, N, \beta, l) \in(0,1)$ such that $D^{\alpha^{\prime}} v \in C^{0, \gamma}\left(\overline{B_{\theta R}^{+}\left(x_{0}\right)} ; \mathbb{R}^{n}\right)$ for every $\alpha^{\prime} \in \mathbb{N}_{0}^{m+1}$ with $\left|\alpha^{\prime}\right| \leq l$ and $\alpha_{m+1}^{\prime}=0$.

Remark 4.2 Henceforth, we assume the conditions on $m$ and $\beta$ from Theorem 4.1. We have restricted to considering $\alpha^{\prime}$ with $\alpha_{m+1}^{\prime}=0$ as (partial) regularity of these derivatives up to the boundary will yield the desired regularity for fractional harmonic maps stated in Theorem 3.8. The main purpose of the theorem is to provide regularity estimates which are uniform up to $\mathcal{O}$; such estimates do not follow from known theory.

Theorem 4.1, combined with the partial regularity theory for harmonic maps yields the following. We use the notation $\mathcal{H}^{t}$ to denote the $t$-dimensional Hausdorff measure, with respect to the Euclidean metric on $\mathbb{R}^{m+1}$, for $t \geq 0$.

Theorem 4.3 Let $v \in \dot{W}_{\beta}^{1,2}\left(\mathbb{R}_{+}^{m+1} ; N\right)$ be a minimiser of $E^{\beta}$ relative to $\mathcal{O}$. There exists sets $\Sigma_{\text {int }} \subset \mathbb{R}_{+}^{m+1}$ and $\Sigma_{\text {bdry }} \subset \mathcal{O}$ such that the following holds. The set $\Sigma_{\text {int }}$ is relatively closed in $\mathbb{R}_{+}^{m+1}$ and has Hausdorff dimension at most $m-2$. The set $\Sigma_{\text {bdry }}$ is relatively closed in $\mathcal{O}$ and $\mathcal{H}^{m+\beta-1}\left(\Sigma_{\text {bdry }}\right)=0$. The set $\Sigma:=\Sigma_{\text {int }} \cup \Sigma_{\text {bdry }}$ is relatively closed in $\mathbb{R}_{+}^{m+1} \cup \mathcal{O}$ and $\mathcal{H}^{m+\beta-1}(\Sigma)=0$. Furthermore, we have $v \in C^{\infty}\left(\mathbb{R}_{+}^{m+1} \backslash \Sigma_{\text {int }} ; N\right), v \in$ $C_{\text {loc }}^{0,1}\left(\left(\mathbb{R}_{+}^{m+1} \cup \mathcal{O}\right) \backslash \Sigma ; N\right)$ and for every multi-index $\alpha^{\prime} \in \mathbb{N}^{m+1}$ with $\alpha_{m+1}^{\prime}=0$ we have 
$D^{\alpha^{\prime}} v \in C_{\text {loc }}^{0,1}\left(\left(\mathbb{R}_{+}^{m+1} \cup \mathcal{O}\right) \backslash \Sigma ; \mathbb{R}^{n}\right)$ and $\nabla D^{\alpha^{\prime}} v \in L_{\text {loc }}^{\infty}\left(\left(\mathbb{R}_{+}^{m+1} \cup \mathcal{O}\right) \backslash \Sigma ; \mathbb{R}^{(m+1) n}\right)$. Finally, for every $\alpha^{\prime} \in \mathbb{N}_{0}^{m+1}$ with $\alpha_{m+1}^{\prime}=0$, we have $x_{m+1}^{\beta} \partial_{m+1} D^{\alpha^{\prime}} v \in C_{\text {loc }}^{0, \gamma}\left(\left(\mathbb{R}_{+}^{m+1} \cup \mathcal{O}\right) \backslash \Sigma ; \mathbb{R}^{(m+1) n}\right)$ for some $\gamma=\gamma\left(m, N, \beta, \alpha^{\prime}\right) \in(0,1)$.

Remark 4.4 The existence and properties of $\Sigma_{\text {int }}$ follows from the theory of Schoen and Uhlenbeck [37].

\subsection{Euler-Lagrange and stationary equations for minimisers}

Minimisers of $E^{\beta}$ relative to $\mathcal{O}$ are critical points of $E^{\beta}$ with respect to outer and inner variations, including those which vary their boundary data in $\mathcal{O}$. As a consequence, they satisfy two systems of partial differential equations which we describe presently.

Let $\psi \in \mathcal{D}_{+}\left(\mathbb{R}_{+}^{m+1} ; \mathbb{R}^{n}\right)$, as defined in Sect. 2.1, with $\psi(\cdot, 0) \in C_{0}^{\infty}\left(\mathcal{O} ; \mathbb{R}^{n}\right)$. For sufficiently small $t$ we define an outer variation of $v$ by $v_{t}=\pi_{N}(v+t \psi) \in N$, where $\pi_{N}$ is the nearest point projection onto $N$. Critical points of $E^{\beta}$ with respect to variations of the form $v_{t}$ satisfy

$$
\int_{\mathbb{R}_{+}^{m+1}} x_{m+1}^{\beta}(\langle\nabla v, \nabla \psi\rangle-\langle\psi, A(v)(\nabla v, \nabla v)\rangle) \mathrm{d} x=0 .
$$

Note that there is a Neumann-type boundary condition implicit in (4.1). In particular, if $v$ is sufficiently smooth in $\mathbb{R}_{+}^{m+1} \cup \mathcal{O}$ we have

$$
x_{m+1}^{\beta} \partial_{m+1} v=0 \text { in } \mathcal{O} .
$$

A (weakly) harmonic map satisfying (4.1) is said to be (weakly) harmonic with respect to the Neumann type boundary condition (4.2).

Define $\Phi_{t}(x)=x+t \phi(x)$ for $x \in \overline{\mathbb{R}_{+}^{m+1}}$, where $\phi \in \mathcal{D}_{+}\left(\mathbb{R}_{+}^{m+1} ; \mathbb{R}^{m+1}\right)$ is such that $\phi(\cdot, 0) \in C_{0}^{\infty}\left(\mathcal{O} ; \partial \mathbb{R}_{+}^{m+1}\right)$ and $|t|$ is small enough to make $\Phi_{t}$ into a diffeomorphism of $\overline{\mathbb{R}_{+}^{m+1}}$ with $\Phi_{t}(\mathcal{O}) \subset \mathcal{O}$. We say $v \in \dot{W}_{\beta}^{1,2}\left(\mathbb{R}_{+}^{m+1} ; N\right)$ is a critical point of the Dirichlet energy corresponding to inner variations $v_{t}:=v \circ \Phi_{t}$, or variations of the independent variable, if $v$ satisfies

$$
\begin{aligned}
& \int_{\mathbb{R}_{+}^{m+1}} \sum_{i=1}^{m+1} \sum_{k=1}^{m+1} x_{m+1}^{\beta}\left(2\left\langle\frac{\partial v}{\partial x_{i}}, \frac{\partial v}{\partial x_{k}}\right\rangle-\delta_{i k}|\nabla v|^{2}\right) \frac{\partial \phi_{k}}{\partial x_{i}} \mathrm{~d} x \\
& =\int_{\mathbb{R}_{+}^{m+1}} \beta x_{m+1}^{\beta-1} \phi_{m+1}|\nabla v|^{2} \mathrm{~d} x
\end{aligned}
$$

for every $\phi$ as above. A weakly harmonic map with respect to the Neumann type boundary condition (4.2) which satisfies (4.3) for every $\phi \in \mathcal{D}_{+}\left(\mathbb{R}_{+}^{m+1} ; \mathbb{R}^{m+1}\right)$ with $\phi(\cdot, 0) \in$ $C_{0}^{\infty}\left(\mathcal{O} ; \partial \mathbb{R}_{+}^{m+1}\right)$ is called weakly stationary harmonic, or stationary harmonic, with respect to the Neumann type boundary condition (4.2).

\subsection{Energy monotonicity}

Stationary harmonic maps satisfy a monotonicity formula for an appropriately scaled version of the energy over balls with closure in $\mathbb{R}_{+}^{m+1}$. This property was proved by Schoen and Uhlenbeck for energy minimisers, see [37, Proposition 2.4], and Price, see the remark after Theorem 1 in [32], for stationary harmonic maps. 
As a consequence of (4.3), we show that stationary harmonic maps with respect to the Neumann-type boundary condition (4.2) satisfy a similar monotonicity formula on half-balls $B_{\rho}^{+}(y)$ with centre $y$ in $\mathcal{O}$ and which satisfy $\overline{\partial^{0} B_{\rho}^{+}(y)}=\overline{B^{m}(y)} \subset \mathcal{O}$. Moreover, we state a version of the formula for balls with closure contained in $\mathbb{R}_{+}^{m+1}$, giving an explicit expression for the factors that the constants involved depend upon.

Lemma 4.5 Suppose $v \in \dot{W}_{\beta}^{1,2}\left(\mathbb{R}_{+}^{m+1} ; N\right)$ is a weakly stationary harmonic map with respect to the Neumann-type boundary condition (4.2). Suppose y in $\mathcal{O}$ and consider $B_{R}^{+}(y)$ with $\overline{\partial^{0} B_{R}^{+}(y)} \subset \mathcal{O}$. Then

$$
\begin{aligned}
& r^{1-m-\beta} \int_{B_{r}^{+}(y)} x_{m+1}^{\beta}|\nabla v|^{2} \mathrm{~d} x-s^{1-m-\beta} \int_{B_{s}^{+}(y)} x_{m+1}^{\beta}|\nabla v|^{2} \mathrm{~d} x \\
& =2 \int_{B_{r}^{+}(y) \backslash B_{s}^{+}(y)} x_{m+1}^{\beta} \frac{|(x-y) \cdot \nabla v|^{2}}{|x-y|^{m+1+\beta}} \mathrm{d} x
\end{aligned}
$$

whenever $0 \leq s \leq r \leq R$ and therefore $\rho \mapsto \rho^{1-m-\beta} \int_{B_{\rho}^{+}(y)} x_{m+1}^{\beta}|\nabla v|^{2} \mathrm{~d} x$ is a nondecreasing function of $\rho$ for $0<\rho \leq R$.

Proof The proof is analogous to that of the monotonicity formula for stationary harmonic maps. We follow [39, Section 2.4] and [29, Lemma 3.3]; we test (4.3) with $\phi(x)=(x-$ $y) \eta(x)$, where $\eta \in C_{0}^{\infty}\left(B_{\rho}(y)\right)$, which yields

$$
\begin{aligned}
& (m-1+\beta) \int_{\mathbb{R}_{+}^{m+1}} x_{m+1}^{\beta}|\nabla v|^{2} \eta \mathrm{d} x+\int_{\mathbb{R}_{+}^{m+1}} x_{m+1}^{\beta}(x-y) \cdot \nabla \eta|\nabla v|^{2} \mathrm{~d} x \\
& \quad=2 \int_{\mathbb{R}_{+}^{m+1}} x_{m+1}^{\beta}\langle(x-y) \cdot \nabla v, \nabla \eta \cdot \nabla v\rangle \mathrm{d} x .
\end{aligned}
$$

Let $\chi \in C_{0}^{\infty}(\mathbb{R} ;[0,1])$ with $\chi(s) \equiv 1$ for $s \geq 1$ and $\chi(s) \equiv 0$ for $s \leq \frac{1}{2}$. The smooth functions defined by $\eta_{j}(x)=\chi(j(\rho-|x-y|))$ are admissible choices for $\eta$ in (4.4) and $\left\{\eta_{j}\right\}_{j \in \mathbb{N}}$ converges pointwise to the indicator function of $B_{\rho}^{+}(y)$. We substitute $\eta_{j}$ for $\eta$ in (4.4) and take the limit as $j \rightarrow \infty$, using Lebesgue's Dominated Convergence and Differentiation Theorems, to see that

$$
\begin{aligned}
& (m-1+\beta) \int_{B_{\rho}^{+}(y)} x_{m+1}^{\beta}|\nabla v|^{2} \mathrm{~d} x-\rho \int_{\partial^{+} B_{\rho}^{+}(y)} x_{m+1}^{\beta}|\nabla v|^{2} \mathrm{~d} S(x) \\
& =-\frac{2}{\rho} \int_{\partial^{+} B_{\rho}^{+}(y)} x_{m+1}^{\beta}|(x-y) \cdot \nabla v|^{2} \mathrm{~d} S(x)
\end{aligned}
$$

for almost every $\rho>0$, where $\mathrm{d} S$ is the Lebesgue measure on $\partial B_{\rho}(y)$. Multiplying the above by the factor $-\rho^{-(\beta+m)}$ and bearing in mind that $\frac{\mathrm{d}}{\mathrm{d} \rho} \int_{B_{\rho}^{+}(y)} x_{m+1}^{\beta}|\nabla v|^{2} \mathrm{~d} x=$ $\int_{\partial^{+} B_{\rho}^{+}(y)} x_{m+1}^{\beta}|\nabla v|^{2} \mathrm{~d} S(x)$ for almost all $\rho>0$, we find

$$
\frac{\mathrm{d}}{\mathrm{d} \rho}\left(\rho^{1-m-\beta} \int_{B_{\rho}^{+}(y)} x_{m+1}^{\beta}|\nabla v|^{2} \mathrm{~d} x\right)=2 \int_{\partial^{+} B_{\rho}^{+}(y)} x_{m+1}^{\beta} \frac{|(x-y) \cdot \nabla v|^{2}}{|x-y|^{m+1+\beta}} \mathrm{d} S(x)
$$

for almost every $\rho>0$. Integrating between $0<s<r$ concludes the proof. 
Remark 4.6 A consequence of Lemma 4.5 is that we can define the density function

$$
\Theta_{v}^{\beta}(y)=\lim _{\rho \rightarrow 0^{+}} \rho^{1-m-\beta} \int_{B_{\rho}^{+}(y)} x_{m+1}^{\beta}|\nabla v|^{2} \mathrm{~d} x
$$

for every $y \in \mathcal{O}$, analogously to Definition 1 in Section 2.5 of [39]. Using Lemma 4.5 we deduce $\Theta_{v}^{\beta}$ is upper semi-continuous in $\mathcal{O}$ for any map $v$ which is weakly stationary harmonic with respect to the Neumann-type boundary condition (4.2).

The following version of the energy monotonicity formula is due to Grosse-Brauckmann [16, Theorem 1]. We do not give a proof, but remark that the explicit form of the constant in the forthcoming formula can be determined using the method of proof of Lemma 4.5.

Lemma 4.7 Suppose $v \in \dot{W}_{\beta}^{1,2}\left(\mathbb{R}_{+}^{m+1} ; N\right)$ is a minimiser of $E^{\beta}$ relative to $\mathcal{O}$. Fix a ball $B_{\rho_{0}}(y)$ with $\overline{B_{\rho_{0}}(y)} \subset \mathbb{R}_{+}^{m+1}$ for some $\rho_{0}>0$ and suppose $r$ and s satisfy $0<s<r<\rho_{0}$. Then

$$
\begin{aligned}
& e^{r C|\beta|} r^{1-m} \int_{B_{r}(y)} x_{m+1}^{\beta}|\nabla v|^{2} \mathrm{~d} x-e^{s C|\beta|} s^{1-m} \int_{B_{s}(y)} x_{m+1}^{\beta}|\nabla v|^{2} \mathrm{~d} x \\
& \geq 2 \int_{B_{r}(y) \backslash B_{s}(y)} x_{m+1}^{\beta} e^{|x-y| C|\beta|} \frac{|(x-y) \cdot \nabla v|^{2}}{|x-y|^{m+1}} \mathrm{~d} x
\end{aligned}
$$

and therefore, for $0<\rho<\rho_{0}, \rho \mapsto e^{\rho C|\beta|} \rho^{1-m} \int_{B_{\rho}(y)} x_{m+1}^{\beta}|\nabla v|^{2} \mathrm{~d} x$ is a non-decreasing function of $\rho$ where $C=\left(y_{m+1}-\rho_{0}\right)^{-1}=\left(\operatorname{dist}\left(B_{\rho_{0}}(y), \partial \mathbb{R}_{+}^{m+1}\right)\right)^{-1}$.

\subsection{A modified lemma of Morrey}

In order to prove Theorem 4.21, in analogy with the regularity theory of harmonic maps, we will show that the re-scaled, scale-invariant energies in the monotonicity formulas in Sect. 4.2 decay slightly faster than implied by the Lemmata as the radius decreases. This will permit the application of a well-known lemma of Morrey, see [29, Lemma 2.1] for example, which is used to derive Hölder continuity from sufficiently fast energy decay. We will reduce the hypothesis of this lemma to similar hypothesis for the re-scaled energies from the monotonicity formula. To this end, we introduce a class of ball with closure in $\mathbb{R}_{+}^{m+1}$ on which the metrics $x_{m+1}^{\alpha} \delta_{i j}$, discussed in Sect. 3 and corresponding weights $x_{m+1}^{\beta}$ are uniformly equivalent to the Euclidean metric and 1 respectively. We also introduce classes of balls and half-balls contained in the interior of a given larger half-ball $B_{R}^{+}\left(x_{0}\right)$ for $R>0$ and $x_{0} \in \partial \mathbb{R}_{+}^{m+1}$.

Define

$$
\mathcal{B}=\left\{B_{\rho}(y) \subset \mathbb{R}_{+}^{m+1}: y_{m+1} \geq 2 \rho\right\} \quad \text { and } \quad \mathcal{B}_{\theta}=\left\{B_{\rho}(y) \subset \mathbb{R}_{+}^{m+1}: y_{m+1} \geq \theta \rho\right\}
$$

for $\theta \geq 2$. Then $\mathcal{B}_{\theta} \subset \mathcal{B}$ and $\mathcal{B}_{2}=\mathcal{B}$. We further define

$$
\mathcal{B}_{\theta}\left(x_{0}, R, r\right)=\left\{B_{\rho}(y) \subset B_{R}^{+}\left(x_{0}\right): y_{m+1} \geq \theta \rho, y \in B_{r}^{+}\left(x_{0}\right)\right\},
$$

omitting the subscript $\theta$ in the case $\theta=2$, and let

$$
\mathcal{B}^{+}\left(x_{0}, R, r\right)=\left\{B_{\rho}^{+}(y) \subset B_{R}^{+}\left(x_{0}\right): y_{m+1}=0,\left|x_{0}-y\right|<r, \rho \leq r\right\} .
$$


Observe that, on any $B_{\rho}(y) \in \mathcal{B}$, we can choose constants $c, C, c_{0}$ and $C_{0}$ independently of $\beta$ such that for every $x \in B_{\rho}(y)$ and $\beta \in(-1,1)$ we have

$$
c y_{m+1}^{\beta} \leq x_{m+1}^{\beta} \leq C y_{m+1}^{\beta} \quad \text { and } \quad c_{0} \leq \frac{\sup _{B_{\rho}(y)} x_{m+1}^{\beta}}{\inf _{B_{\rho}(y)} x_{m+1}^{\beta}} \leq C_{0} .
$$

Lemma 4.8 Let $\gamma>0, x_{0} \in \partial \mathbb{R}_{+}^{m+1}, a>0, \theta_{1} \geq 2$ and $\theta_{2} \leq \frac{1}{2}$. Define $\theta=\frac{\theta_{2}}{2 \theta_{1}}$. Then there exists a constant $C_{0}=C_{0}\left(m, \gamma, \theta_{1}, \beta\right)$ such that if $v \in W_{\beta}^{1,2}\left(B_{R}^{+}\left(x_{0}\right) ; \mathbb{R}^{n}\right)$ with

$$
r^{-2 m} \int_{B}\left|x_{m+1}\right|^{-\beta} \mathrm{d} x \int_{B}|\nabla v|^{2}\left|x_{m+1}\right|^{\beta} \mathrm{d} x \leq a r^{\gamma}
$$

for every $B=B_{r}^{+}(y) \in \mathcal{B}^{+}\left(x_{0}, R, \theta_{2} R\right)$ and every $B=B_{r}(y) \in \mathcal{B}_{\theta_{1}}\left(x_{0}, R, \theta_{2} R\right)$, then for almost every $x_{1}, x_{2} \in B_{\theta R}^{+}\left(x_{0}\right)$,

$$
\left|v\left(x_{1}\right)-v\left(x_{2}\right)\right| \leq C_{0} a^{\frac{1}{2}}\left|x_{1}-x_{2}\right|^{\frac{\gamma}{2}} .
$$

Proof Let $B_{r}(y) \subset B_{2 \theta R}\left(x_{0}\right)$ with $y_{m+1} \geq 0, y \in B_{\theta R}\left(x_{0}\right)$ and $r \leq \theta R$, for a $\gamma \in(0,1)$, an $a>0$ and $\theta$ as specified. Such a $B_{r}(y)$ must satisfy either $B_{r}(y) \in \mathcal{B}_{\theta_{1}}\left(x_{0}, 2 \theta R, \theta R\right)$ or $B_{r}(y) \notin \mathcal{B}_{\theta_{1}}\left(x_{0}, 2 \theta R, \theta R\right)$. We consider these cases in turn and we work with the even reflection of $v$ with respect to $\partial \mathbb{R}_{+}^{m+1}$, which we do not relabel and which is in $W_{\beta}^{1,2}\left(B_{R}\left(x_{0}\right) ; \mathbb{R}^{n}\right)$.

Suppose $B_{r}(y) \in \mathcal{B}_{\theta_{1}}\left(x_{0}, 2 \theta R, \theta R\right)$ with $r \leq \theta R$. Then $B_{r}(y) \in \mathcal{B}_{\theta_{1}}\left(x_{0}, R, \theta_{2} R\right)$ and an application of Hölder's inequality and the assumptions of the lemma yields

$$
r^{-m} \int_{B_{r}(y)}|\nabla v| \mathrm{d} x \leq\left(r^{-2 m} \int_{B_{r}(y)}\left|x_{m+1}\right|^{-\beta} \mathrm{d} x \int_{B_{r}(y)}|\nabla v|^{2}\left|x_{m+1}\right|^{\beta} \mathrm{d} x\right)^{\frac{1}{2}} \leq a^{\frac{1}{2}} r^{\frac{\gamma}{2}} .
$$

Now suppose $B_{r}(y) \notin \mathcal{B}_{\theta_{1}}\left(x_{0}, 2 \theta R, \theta R\right)$ and $r \leq \theta R$. In this case, since $B_{r}(y) \subset$ $B_{2 \theta R}\left(x_{0}\right)$ and $y \in B_{\theta R}\left(x_{0}\right)$ by assumption, we must have $y_{m+1}<\theta_{1} r$. Hence $y_{m+1}-r<\zeta r$, where $\zeta \geq 1$ is such that $\theta_{1}=\zeta+1$, and thus $B_{r}(y) \subset B_{(2+\zeta) r}\left(y_{0}\right)$ where $y_{0}=y-\left(0, y_{m+1}\right)$. We observe that $B_{(2+\zeta) r}^{+}\left(y_{0}\right) \in \mathcal{B}^{+}\left(x_{0}, R, \theta_{2} R\right)$. Therefore, defining $s=(2+\zeta) r$ and using the assumptions of the lemma, the symmetry of $v$ and applying Hölder's inequality, we find

$$
\begin{aligned}
r^{-m} \int_{B_{r}(y)}|\nabla v| \mathrm{d} x & \leq r^{-m} \int_{B_{s}\left(y_{0}\right)}|\nabla v| \mathrm{d} x \\
& =2 r^{-m} \int_{B_{s}^{+}\left(y_{0}\right)}|\nabla v| \mathrm{d} x \\
& \leq 2(2+\zeta)^{m}\left(s^{-2 m} \int_{B_{s}^{+}\left(y_{0}\right)}\left|x_{m+1}\right|^{-\beta} \mathrm{d} x \int_{B_{s}^{+}\left(y_{0}\right)}|\nabla v|^{2}\left|x_{m+1}\right|^{\beta} \mathrm{d} x\right)^{\frac{1}{2}} \\
& \leq 2(2+\zeta)^{m+\frac{\gamma}{2}} a^{\frac{1}{2}} r^{\frac{\gamma}{2}}
\end{aligned}
$$

Since $v$ is even with respect to $\partial \mathbb{R}_{+}^{m+1}$, we deduce that either (4.8) or (4.9) holds on any $B_{r}(y) \subset B_{2 \theta R}\left(x_{0}\right)$ with $y \in B_{\theta R}\left(x_{0}\right)$ and $r \leq \theta R$. Hence we have established that the hypothesis of the decay lemma of Morrey hold on $B_{2 \theta R}\left(x_{0}\right)$, see [29, Lemma 2.1]. An application of this lemma concludes the proof. 


\subsection{Interior estimates for Hölder continuity}

Using the regularity theory of Schoen and Uhlenbeck [37] and Schoen [38], we show that minimisers $v \in \dot{W}_{\beta}^{1,2}\left(\mathbb{R}_{+}^{m+1} ; N\right)$ of $E^{\beta}$ relative to $\mathcal{O}$ essentially satisfy (4.7) in Lemma 4.8, provided the scale-invariant energy $R^{1-m-\beta} \int_{B_{R}^{+}\left(x_{0}\right)} x_{m+1}^{\beta}|\nabla v|^{2} \mathrm{~d} x$ is sufficiently small. To this end, we show that the preceding scale-invariant energy $B_{R}^{+}\left(x_{0}\right)$ controls the scaleinvariant Euclidean energy on a class of ball with closure in $B_{R}^{+}\left(x_{0}\right)$. We also recall the relevant theory from [37,38, Sections 1,2 and 3], stating the results in our context with slightly different notation. Our goal is to prove the following.

Lemma 4.9 Suppose $v \in \dot{W}_{\beta}^{1,2}\left(\mathbb{R}_{+}^{m+1} ; N\right)$ is a minimiser of $E^{\beta}$ relative to $\mathcal{O}$. Let $B_{R}^{+}\left(x_{0}\right)$ be a half-ball with $R \leq 1$ and $\overline{\partial^{0} B_{R}^{+}\left(x_{0}\right)} \subset \mathcal{O}$. There exists an $\varepsilon_{0}=\varepsilon_{0}(m, N)>0$, a $\theta=\theta(m, N) \geq 2$ and a positive $C=C(m, N)$ such that if

$$
R^{1-m-\beta} \int_{B_{R}^{+}\left(x_{0}\right)} x_{m+1}^{\beta}|\nabla v|^{2} \mathrm{~d} x \leq \varepsilon_{0}
$$

then

$$
\rho^{1-m} \int_{B_{\rho}(y)}|\nabla v|^{2} \mathrm{~d} x \leq C\left(\frac{\rho}{r}\right)^{\gamma} r^{1-m} \int_{B_{r}(y)}|\nabla v|^{2} \mathrm{~d} x
$$

on every $B_{r}(y) \in \mathcal{B}_{\theta}\left(x_{0}, R, \frac{R}{3}\right)$ for $0<\rho \leq r$ and $a \gamma=\gamma(m, N) \in(0,1)$.

To establish the preceding lemma, we observe the following relationship between the scale-invariant energy on $B_{R}^{+}\left(x_{0}\right)$ and the scale-invariant Euclidean energy on a class of ball with closure in $B_{R}^{+}\left(x_{0}\right)$.

Lemma 4.10 Suppose $v \in \dot{W}_{\beta}^{1,2}\left(\mathbb{R}_{+}^{m+1} ; N\right)$ is a minimiser of $E^{\beta}$ relative to $\mathcal{O}$. Let $B_{R}^{+}\left(x_{0}\right)$ be a half-ball with $\overline{\partial^{0} B_{R}^{+}\left(x_{0}\right)} \subset \mathcal{O}$ and suppose $B_{\rho}(y) \in \mathcal{B}\left(x_{0}, R, \frac{R}{3}\right)$. Then there is a constant $C=C(m)$ such that

$$
\rho^{1-m} \int_{B_{\rho}(y)}|\nabla v|^{2} \mathrm{~d} x \leq C R^{1-m-\beta} \int_{B_{R}^{+}\left(x_{0}\right)} x_{m+1}^{\beta}|\nabla v|^{2} \mathrm{~d} x .
$$

Proof Notice that any ball $B_{\rho}(y) \in \mathcal{B}$ satisfies $B_{\rho}(y) \subset B_{\frac{y_{m+1}}{2}}(y)$ so we can choose the scaling factor $e^{\frac{2|\beta| \rho}{y_{m+1}}}$ in Lemma 4.7. Furthermore, $e^{\frac{2|\beta| \rho}{y_{m+1}}} \leq e$ since $y_{m+1} \geq 2 \rho$ and $\beta \in$ $(-1,1)$. Hence, using (4.6) and applying Lemma 4.7, we find

$$
\begin{aligned}
\rho^{1-m} \int_{B_{\rho}(y)}|\nabla v|^{2} \mathrm{~d} x & \leq C y_{m+1}^{-\beta} e^{\frac{2|\beta| \rho}{y_{m+1}}} \rho^{1-m} \int_{B_{\rho}(y)} x_{m+1}^{\beta}|\nabla v|^{2} \mathrm{~d} x \\
& \leq C\left(\frac{y_{m+1}}{2}\right)^{1-m-\beta} \int_{B_{\frac{y_{m+1}}{2}}(y)} x_{m+1}^{\beta}|\nabla v|^{2} \mathrm{~d} x .
\end{aligned}
$$

Let $y=\left(y_{1}, \ldots, y_{m+1}\right)$ and $y^{+}=\left(y_{1}, \ldots, y_{m}, 0\right)$. Note that $B_{\frac{y_{m+1}}{2}}(y) \subset B_{\frac{3 y_{m+1}}{2}}^{+}\left(y^{+}\right)$and, since $B_{\rho}(y) \in \mathcal{B}\left(x_{0}, R, \frac{R}{3}\right)$, we have $y \in B_{\frac{R}{3}}^{+}\left(x_{0}\right)$ and $B_{\frac{3 y_{m+1}}{2}}^{+}\left(y^{+}\right) \subset B_{\frac{R}{2}}^{+}\left(y^{+}\right) \subset B_{R}^{+}\left(x_{0}\right)$. Using these facts we apply Lemma 4.5 to see that

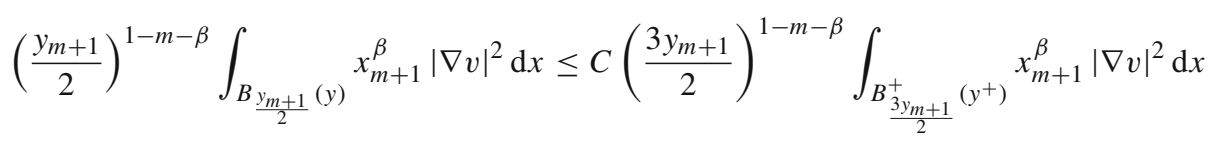




$$
\begin{aligned}
& \leq C\left(\frac{R}{2}\right)^{1-m-\beta} \int_{B_{\frac{R}{2}}^{+}\left(y^{+}\right)} x_{m+1}^{\beta}|\nabla v|^{2} \mathrm{~d} x \\
& \leq C R^{1-m-\beta} \int_{B_{R}^{+}\left(x_{0}\right)} x_{m+1}^{\beta}|\nabla v|^{2} \mathrm{~d} x
\end{aligned}
$$

where $C=C(m)$. The combination of (4.12) and (4.13) yields (4.11).

The maps considered in $[37,38]$ belong to

$$
W^{1,2}(\Omega ; N)=\left\{v \in W^{1,2}\left(\Omega ; \mathbb{R}^{n}\right): v(x) \in N \text { for almost every } x \in B_{r}(y)\right\},
$$

for open $\Omega \subset \mathbb{R}_{+}^{m+1}$. Consider the compact Riemannian manifold $\overline{B_{1}(0)}$ with metric $\tilde{g}$. Recall from the introduction that the Dirichlet energy functional on $\overline{B_{1}(0)}$ is given by

$$
E_{\tilde{g}}(v)=\int_{B_{1}(0)}|\nabla v|_{\tilde{g}}^{2} \sqrt{\operatorname{det}(\tilde{g})} \mathrm{d} x .
$$

A minimiser of $E_{\tilde{g}}$ with fixed boundary data is defined in Section 1 of [37] as follows.

Definition 4.11 Any $v \in W^{1,2}\left(B_{1}(0) ; N\right)$ is an $E_{\tilde{g}}$ minimising map if it satisfies $E_{\tilde{g}}(v) \leq$ $E_{\tilde{g}}(w)$ for any $w \in W^{1,2}\left(B_{1}(0) ; N\right)$ with $v-w \in W_{0}^{1,2}\left(\overline{B_{1}(0)} ; \mathbb{R}^{n}\right)$.

The metric $\tilde{g}$ is assumed to be of class $C^{2}$ on $\overline{B_{1}(0)}$. For $\Lambda>0$ denote by $\mathscr{E}_{\Lambda}$ the class of functionals $E_{\tilde{g}}$ on $\overline{B_{1}(0)}$ with metric $\tilde{g}$ such that $\tilde{g}_{i j}(0)=\delta_{i j}$ and

$$
\sum_{i, j, k}\left|\partial_{k} \tilde{g}_{i j}\right| \leq \Lambda
$$

If $v$ is $E_{\tilde{g}}$-minimising with $E_{\tilde{g}} \in \mathscr{E}_{\Lambda}$ then we say $v \in \mathscr{H}_{\Lambda}$.

Schoen and Uhlenbeck [37] proved their $\varepsilon$-regularity theorem for minimisers of functionals of the form $\tilde{E}_{\tilde{g}}+F$, where $F$ gives rise to terms in the Euler-Lagrange equations which are lower order than those coming from the energy. We state the result of their theorem with $F=0$. The following Lemma is Theorem 3.1 in [37].

Lemma 4.12 There exists $\varepsilon=\varepsilon(m, N)>0$ such that if $v \in \mathscr{H}_{\Lambda}, \Lambda \leq \varepsilon$ and $\int_{B_{1}(0)}|\nabla v|^{2} \mathrm{~d} x \leq \varepsilon$, then $v$ is Hölder continuous in $B_{\frac{1}{2}}(0)$ and

$$
\left|v\left(x_{1}\right)-v\left(x_{2}\right)\right| \leq C\left|x_{1}-x_{2}\right|^{\gamma}
$$

for constants $C=C(m, N)$ and $\gamma=\gamma(m, N) \in(0,1)$ and every $x_{1}, x_{2} \in B_{\frac{1}{2}}(0)$.

It is well known that continuous weakly harmonic maps are smooth, see [22] for example. It is more readily shown that Hölder continuous harmonic maps are smooth; this is the content of the following lemma, which is Lemma 3.1 of [38].

Lemma 4.13 Consider a ball $B_{r}(y) \subset \mathbb{R}_{+}^{m+1}$ and suppose $v \in W^{1,2}\left(B_{r}(y) ; N\right)$ is a weakly harmonic map which is Hölder continuous on $B_{r}(y)$. Then $v$ is smooth on $B_{r}(y)$.

The final lemma we will need is Theorem 2.2 from [38].

Lemma 4.14 Let $v \in C^{2}\left(B_{r}(0) ; N\right)$ and $\tilde{g}$ be a Riemannian metric on $B_{r}(0)$. Suppose $v$ is harmonic with respect to $\tilde{g}$ in $B_{r}(0)$ and $\tilde{g}$ satisfies $\left|\partial_{k} \tilde{g}_{i j}\right| \leq \Lambda r^{-1}$ for $i, j, k=1, \ldots, m+1$ 
and $\Lambda^{-1}\left(\delta_{i j}\right) \leq\left(\tilde{g}_{i j}\right) \leq \Lambda\left(\delta_{i j}\right)$ in the sense oftensors, where $\delta_{i j}=1$ when $i=j$ and $\delta_{i j}=0$ otherwise. Then there exists an $\varepsilon=\varepsilon(\Lambda, m, N)>0$ such that if

$$
r^{1-m} \int_{B_{r}(0)}|\nabla v|_{\tilde{g}}^{2}(\operatorname{det}(\tilde{g}))^{\frac{1}{2}} \mathrm{~d} x \leq \varepsilon
$$

then

$$
\sup _{B_{\frac{r}{2}}(0)}|\nabla v|_{\tilde{g}}^{2} \leq C r^{-(1+m)} \int_{B_{r}(0)}|\nabla v|_{\tilde{g}}^{2}(\operatorname{det}(\tilde{g}))^{\frac{1}{2}} \mathrm{~d} x
$$

for a constant $C=C(\Lambda, m, N)$.

Proof of Lemma 4.9 Suppose $R^{1-m-\beta} \int_{B_{R}^{+}\left(x_{0}\right)} x_{m+1}^{\beta}|\nabla v|^{2} \mathrm{~d} x \leq \varepsilon_{0}$ for an $\varepsilon_{0}>0$ to be chosen small and let $\varepsilon$ be the number from Lemma 4.12 .

Recall the metric $g$ given in Euclidean coordinates by $g:=x_{m+1}^{\alpha} \delta_{i j}$ and define $\hat{g}$ on $\overline{B_{1}(0)}$ by

$$
\hat{g}_{i j}(x)=\delta_{i j}\left(1+r y_{m+1}^{-1} x_{m+1}\right)^{\alpha}=y_{m+1}^{-\alpha} g_{i j}(r x+y) .
$$

The energy corresponding to $\hat{g}$ is

$$
E_{\hat{g}}(\hat{v})=\frac{1}{2} \int_{B_{1}(0)}\left(1+r y_{m+1}^{-1} x_{m+1}\right)^{\beta}|\nabla \hat{v}|^{2} \mathrm{~d} x
$$

for maps $\hat{v} \in W^{1,2}\left(B_{1}(0) ; \mathbb{R}^{n}\right)$. Since $B_{r}(y) \in B_{\theta}\left(x_{0}, R, \frac{R}{3}\right)$ for a $\theta \geq 2$ to be chosen, we have $B_{r}(y) \in \mathcal{B}$. Hence, using (4.6) and noting that $\beta=\alpha\left(\frac{m-1}{2}\right) \in(-1,1)$, we find constants $c, C$ depending only on $m$ such that

$$
c \leq\left(1+r y_{m+1}^{-1} x_{m+1}\right)^{\beta} \leq C \quad \text { and } \quad c \leq \hat{g}_{i j}(x) \leq C .
$$

We note that $\partial_{k} \hat{g}=0$ for $k \neq m+1$ and, again using (4.6), we calculate

$$
\left|\partial_{m+1}\left(1+r y_{m+1}^{-1} x_{m+1}\right)^{\alpha}\right|=r y_{m+1}^{-1}|\alpha|\left|\left(1+r y_{m+1}^{-1} x_{m+1}\right)^{\alpha-1}\right| \leq C r y_{m+1}^{-1}
$$

where $C$ is chosen independently of $\alpha$. Hence, if we set $\theta=\theta(m, N) \geq \max \left\{2,(m+1) C \varepsilon^{-1}\right\}$ then we conclude that

$$
\sum_{i, j, k}\left|\partial_{k} \tilde{g}_{i j}\right|=\sum_{i=1}^{m+1}\left|\partial_{m+1} \tilde{g}_{i i}\right| \leq \varepsilon .
$$

We assume the preceding choice of $\theta$ henceforth so that (4.17) holds on any $B_{r}(y) \in$ $\mathcal{B}_{\theta}\left(x_{0}, R, \frac{R}{3}\right)$.

Define $v_{r, y}(x)=v(r x+y)$ for $x \in B_{1}(0)$. Lemmata 2.1 and 2.3 imply $\dot{W}_{\beta}^{1,2}\left(\mathbb{R}_{+}^{m+1} ; N\right) \hookrightarrow$ $W^{1,2}\left(B_{r}(y) ; N\right)$ for every $B_{r}(y)$ with $\overline{B_{r}(y)} \subset \mathbb{R}_{+}^{m+1}$, regardless of $\beta \in(-1,1)$. A change of variables then yields $v_{r, y} \in W^{1,2}\left(B_{1}(0) ; N\right)$. Furthermore, since $v$ is a minimiser of $E^{\beta}$ relative to $\mathcal{O}$ we readily calculate that $v_{r, y}$ is a minimiser of $E_{\hat{g}}$ in the sense of Definition 4.11 on $B_{1}(0)$, that is, $v_{r, y}$ minimises $E_{\hat{g}}$ among all maps in $W^{1,2}\left(B_{1}(0) ; N\right)$ with the same boundary values as $v_{r, y}$. 
Our considerations so far imply that if $B_{r}(y) \in \mathcal{B}_{\theta}\left(x_{0}, R, \frac{R}{3}\right)$, for our preceding choice of $\theta$, then $v_{r, y} \in \mathscr{H}_{\varepsilon}$. Let $C$ be the constant from Lemma 4.10 and suppose that $\varepsilon_{0} \leq C^{-1} \varepsilon$. An application of this lemma, combined with a change of variables, yields

$$
\int_{B_{1}(0)}\left|\nabla v_{r, y}\right|^{2} \mathrm{~d} x=r^{1-m} \int_{B_{r}(y)}|\nabla v|^{2} \mathrm{~d} x \leq C R^{1-m-\beta} \int_{B_{R}^{+}\left(x_{0}\right)} x_{m+1}^{\beta}|\nabla v|^{2} \mathrm{~d} x \leq C \varepsilon_{0} \leq \varepsilon .
$$

This holds for every $B_{r}(y) \in \mathcal{B}_{\theta}\left(x_{0}, R, \frac{R}{3}\right)$. We may therefore apply Lemma 4.12 to $v_{r, y}$ to deduce that it is Hölder continuous in $B_{\frac{1}{2}}(0)$. Re-scaling implies $v$ is Hölder continuous in every $B_{\frac{r}{2}}(y) \in \mathcal{B}_{\theta}\left(x_{0}, R, \frac{R}{3}\right)$, or equivalently $v$ is Hölder continuous in every $B_{r}(y) \in$ $\mathcal{B}_{2 \theta}\left(x_{0}, R, \frac{R}{3}\right)$.

Since $v$ is weakly harmonic in $\mathbb{R}_{+}^{m+1}$ with respect to the metric represented by $x_{m+1}^{\alpha} \delta_{i j}$ (where $\beta=\alpha \frac{m-1}{2}$ ), it is weakly harmonic with respect to $x_{m+1}^{\alpha} \delta_{i j}$ on every $B_{r}(y)$ with $\overline{B_{r}(y)} \subset \mathbb{R}_{+}^{m+1}$. Thus it follows from Lemma 4.13 that $v$ is smooth in each $B_{r}(y) \in$ $\mathcal{B}_{2 \theta}\left(x_{0}, R, \frac{R}{3}\right)$, which holds if and only if each $v_{r, y}$ corresponding to such a $B_{r}(y)$ is smooth in $B_{1}(0)$. We further deduce that $v_{r, y}$ is harmonic in $B_{1}(0)$ with respect to $\hat{g}$ using the chain rule. Moreover, it follows from (4.16) and (4.17) that $\hat{g}$ satisfies the assumptions required of the metric in Lemma 4.14. We combine (4.16) with (4.18) to see that

$$
\int_{B_{1}(0)}\left(1+r y_{m+1}^{-1} x_{m+1}\right)^{\beta}\left|\nabla v_{r, y}\right|^{2} \mathrm{~d} x \leq C \int_{B_{1}(0)}\left|\nabla v_{r, y}\right|^{2} \mathrm{~d} x \leq C \varepsilon_{0} .
$$

In addition to our previous stipulation for $\varepsilon_{0}$, we further require that $\varepsilon_{0} \leq \frac{\varepsilon_{1}}{C}$ where $\varepsilon_{1}$ is the number from Lemma 4.14. We apply this lemma, recalling $\beta=\alpha\left(\frac{m-1}{2}\right)$ and $B_{r}(y) \in \mathcal{B}$, to see that

$$
\begin{aligned}
r^{2} \sup _{B_{\frac{r}{2}}(y)}\left(x_{m+1} y_{m+1}^{-1}\right)^{\beta}|\nabla v|^{2} & =\sup _{B_{\frac{1}{2}}(0)}\left(1+r y_{m+1}^{-1} x_{m+1}\right)^{\beta}|\nabla v r, y|^{2} \\
& \leq C \sup _{B_{\frac{1}{2}}(0)}\left(1+r y_{m+1}^{-1} x_{m+1}\right)^{-\alpha}\left|\nabla v_{r, y}\right|^{2} \\
& \leq C \int_{B_{1}(0)}\left(1+r y_{m+1}^{-1} x_{m+1}\right)^{\beta}\left|\nabla v_{r, y}\right|^{2} \mathrm{~d} x \\
& =C r^{1-m} \int_{B_{r}(y)}\left(x_{m+1} y_{m+1}^{-1}\right)^{\beta}|\nabla v|^{2} \mathrm{~d} x .
\end{aligned}
$$

As a result, for any $\sigma \in\left(0, \frac{1}{2}\right]$ we have

$$
\begin{aligned}
& (\sigma r)^{1-m} \int_{B_{\sigma r}(y)}\left(x_{m+1} y_{m+1}^{-1}\right)^{\beta}|\nabla v|^{2} \mathrm{~d} x \\
& \quad \leq C \sigma^{2} r^{1-m} \int_{B_{r}(y)}\left(x_{m+1} y_{m+1}^{-1}\right)^{\beta}|\nabla v|^{2} \mathrm{~d} x .
\end{aligned}
$$

Choose $\sigma$ such that $\sigma^{2} \leq \frac{1}{2 C}$ and let $\rho \leq r$. Then

$$
\begin{aligned}
& e^{\frac{|\beta| \sigma r}{y_{m+1}-r}}(\sigma r)^{1-m} \int_{B_{\sigma r}(y)}\left(x_{m+1} y_{m+1}^{-1}\right)^{\beta}|\nabla v|^{2} \mathrm{~d} x \\
& \leq \frac{1}{2} e^{\frac{|\beta| r}{y_{m+1}-r}} r^{1-m} \int_{B_{r}(y)}\left(x_{m+1} y_{m+1}^{-1}\right)^{\beta}|\nabla v|^{2} \mathrm{~d} x
\end{aligned}
$$


on every $B_{r}(y) \in \mathcal{B}_{2 \theta}\left(x_{0}, R, \frac{R}{3}\right)$. The map $\rho \mapsto e^{\frac{|\beta| \rho}{y_{m+1}-r}} \rho^{1-m} \int_{B_{\rho}(y)}\left(x_{m+1} y_{m+1}^{-1}\right)^{\beta}|\nabla v|^{2} \mathrm{~d} x$ is non-decreasing in $\rho$ for $\rho \leq r$ as a result of Lemma 4.7. Lemma 8.23 of [15] hence implies

$$
\begin{aligned}
& e^{\frac{|\beta| \rho}{y_{m+1}-r}} \rho^{1-m} \int_{B_{\rho}(y)}\left(x_{m+1} y_{m+1}^{-1}\right)^{\beta}|\nabla v|^{2} \mathrm{~d} x \\
& \leq C\left(\frac{\rho}{r}\right)^{\gamma} e^{\frac{|\beta| r}{y_{m+1}-r}} r^{1-m} \int_{B_{r}(y)}\left(x_{m+1} y_{m+1}^{-1}\right)^{\beta}|\nabla v|^{2} \mathrm{~d} x
\end{aligned}
$$

for every $\rho \leq r$ on every $B_{r}(y) \in \mathcal{B}_{2 \theta}\left(x_{0}, R, \frac{R}{3}\right)$. It follows that

$$
\rho^{1-m} \int_{B_{\rho}(y)}|\nabla v|^{2} \mathrm{~d} x \leq C\left(\frac{\rho}{r}\right)^{\gamma} r^{1-m} \int_{B_{r}(y)}|\nabla v|^{2} \mathrm{~d} x
$$

on every $B_{r}(y) \in \mathcal{B}_{2 \theta}\left(x_{0}, R, \frac{R}{3}\right)$ for $0<\rho \leq r$. This concludes the proof.

\subsection{A modified lemma of Luckhaus}

Here we begin our construction of comparison maps. We prove a partial extension (to the particular case of our degenerate/singular metrics $x_{m+1}^{\alpha} \delta_{i j}$ ) of a lemma of Luckhaus, Lemma 3 in [23], as presented in Lemma 1 Section 2.6 of [39].

Let $\mathbb{S}^{m} \subset \mathbb{R}^{m+1}$ denote the $m$ dimensional unit sphere, centred at the origin and equipped with the metric induced by the Euclidean metric on $\mathbb{R}^{m+1}$. Define $\mathbb{S}_{+}^{m}=\mathbb{S}^{m} \cap \mathbb{R}_{+}^{m+1}$ with the metric induced from $\mathbb{S}^{m}$. We let $\omega$ denote a point in $\mathbb{S}^{m} \subset \mathbb{R}^{m+1}$ or $\mathbb{S}_{+}^{m} \subset \mathbb{R}_{+}^{m+1}$ and write $\mathrm{d} \omega$ for the volume element corresponding to the induced metric. Recall the notation $\partial^{+} \Omega=$ $\partial \Omega \cap \mathbb{R}_{+}^{m+1}$ for $\Omega \subset \mathbb{R}^{m+1}$ and $Q_{r}(y)=\left\{x \in \mathbb{R}^{m+1}:\left|x_{i}-y_{i}\right|<r, i=1, \ldots, m+1\right\}$ for $y \in \mathbb{R}^{m+1}$. We also write $Q_{r}^{+}(y)=Q_{r}(y) \cap \mathbb{R}_{+}^{m+1}$ for $y \in \partial \mathbb{R}_{+}^{m+1}$.

In order to state the modified Luckhaus lemma precisely we introduce the notion of a Sobolev space for functions whose domain is either $\mathbb{S}^{m}$ or $\mathbb{S}_{+}^{m}$.

Definition 4.15 Let $\varepsilon>0$ and $\rho>0$. Suppose $S=\rho \mathbb{S}^{m}$ and $V_{\varepsilon}=B_{\rho+\varepsilon}(0) \backslash B_{\rho-\varepsilon}(0)$ or $S=\rho \mathbb{S}_{+}^{m}$ and $V_{\varepsilon}=B_{\rho+\varepsilon}^{+}(0) \backslash B_{\rho-\varepsilon}^{+}(0)$. An element $v \in L_{\beta}^{2}\left(S ; \mathbb{R}^{n}\right)$ is said to be in $W_{\beta}^{1,2}\left(S ; \mathbb{R}^{n}\right)$ if the map $v\left(\rho \frac{x}{|x|}\right) \in W_{\beta}^{1,2}\left(V_{\varepsilon} ; \mathbb{R}^{n}\right)$ for some $\varepsilon>0$. An element $v \in L_{\beta}^{2}(S \times$ $\left.[a, b] ; \mathbb{R}^{n}\right)$, with $a<b$ real numbers, is said to be in $W_{\beta}^{1,2}\left(S \times[a, b] ; \mathbb{R}^{n}\right)$ if the map $v\left(\rho \frac{x}{|x|}, s\right) \in W_{\beta}^{1,2}\left(V_{\varepsilon} \times[a, b] ; \mathbb{R}^{n}\right)$ for some $\varepsilon>0$. If $N \subset \mathbb{R}^{n}$ is compact, we say $v$ is in $W_{\beta}^{1,2}(S ; N)$ or $W_{\beta}^{1,2}(S \times[a, b] ; N)$ if $v$ is in $W_{\beta}^{1,2}\left(S ; \mathbb{R}^{n}\right)$ or $W_{\beta}^{1,2}\left(S \times[a, b] ; \mathbb{R}^{n}\right)$ respectively and $v(x) \in N$ for almost every $x \in S$.

We now state our version of the Luckhaus lemma.

Lemma 4.16 Let $m \geq 2$ and $\beta \in(-1,1)$. Let $N$ be a compact subset of $\mathbb{R}^{n}$ and suppose $u, v \in W_{\beta}^{1,2}\left(\mathbb{S}_{+}^{m} ; N\right)$. Then for all $\varepsilon \in(0,1)$ there is a $w \in W_{\beta}^{1,2}\left(\mathbb{S}_{+}^{m} \times[0, \varepsilon] ; \mathbb{R}^{n}\right)$ such that $w$ agrees with $u$ on $\mathbb{S}_{+}^{m} \times\{0\}$ and $v$ on $\mathbb{S}_{+}^{m} \times\{\varepsilon\}$ in the sense of traces and which satisfies the following. Let $\bar{D}$ be the gradient on $\mathbb{S}_{+}^{m} \times[0, \varepsilon]$ and $D$ the gradient on $\mathbb{S}_{+}^{m}$. Then $w=w(\omega, s)$ satisfies

$$
\begin{aligned}
& \int_{\mathbb{S}_{+}^{m} \times[0, \varepsilon]} \omega_{m+1}^{\beta}|\bar{D} w|^{2} \mathrm{~d} \omega \mathrm{d} s \\
& \quad \leq C_{1} \varepsilon \int_{\mathbb{S}_{+}^{m}} \omega_{m+1}^{\beta}\left(|D u|^{2}+|D v|^{2}\right) \mathrm{d} \omega+\frac{C_{1}}{\varepsilon} \int_{\mathbb{S}_{+}^{m}} \omega_{m+1}^{\beta}|u-v|^{2} \mathrm{~d} \omega
\end{aligned}
$$


where $C_{1}=C_{1}(m, \beta)$. Furthermore, $w$ satisfies

$$
\begin{aligned}
& \operatorname{dist}^{2}(w(\omega, s), N) \\
& \leq \frac{C_{2}}{\varepsilon^{m+\frac{\beta}{2}+\frac{|\beta|}{2}}}\left(\int_{\mathbb{S}_{+}^{m}} \omega_{m+1}^{\beta}\left(|D u|^{2}+|D v|^{2}\right) \mathrm{d} \omega\right)^{\frac{1}{q}}\left(\int_{\mathbb{S}_{+}^{m}} \omega_{m+1}^{\beta}|u-v|^{2} \mathrm{~d} \omega\right)^{1-\frac{1}{q}} \\
& \quad+\frac{C_{2}}{\varepsilon^{m+1+\frac{\beta}{2}+\frac{|\beta|}{2}}} \int_{\mathbb{S}_{+}^{m}} \omega_{m+1}^{\beta}|u-v|^{2} \mathrm{~d} \omega
\end{aligned}
$$

for almost every $(\omega, s) \in \mathbb{S}_{+}^{m} \times[0, \varepsilon]$ where $C_{2}=C_{2}(m, \beta)$ and $q$ satisfies the following. If $\beta \in(-1,0]$ then (4.21) holds for $q=2$. If $\beta \in(0,1)$, for any $p \in\left(1, \frac{2}{1+\beta}\right)$, there exists $q \in\{2, p\}$ such that (4.21) holds.

Our proof of Lemma 4.16 follows the proof, given in Section 2.12.2 of [39], of Lemma 1 in Section 2.6 of [39].

\subsection{Absolute continuity properties of functions in $W_{\beta}^{1,2}$}

We recall the discussion in [39, Section 2.12.1] regarding the absolute continuity properties of $W^{1, p}$ functions, which are inherited by $W_{\beta}^{1,2}$ functions in view of Lemma 2.1. Let $\mathcal{H}^{t}$ denote the $t$-dimensional Hausdorff measure with respect to the Euclidean metric. Consider a rectangle $Q \subset \overline{\mathbb{R}_{+}^{m+1}}$ of the form $Q=\left[a_{1}, b_{1}\right] \times \cdots \times\left[a_{m+1}, b_{m+1}\right]$ where $a_{i}<b_{i}$. Suppose $v \in W_{\beta}^{1,2}\left(Q ; \mathbb{R}^{n}\right)$ with $\beta \in(-1,1)$. It follows from Lemma 2.1 that if $a_{m+1}>0$ then $Q \subset \mathbb{R}_{+}^{m+1}$ and $\left.v\right|_{Q} \in W^{1,2}\left(Q ; \mathbb{R}^{n}\right)$. Lemma 2.1 also implies that if $a_{m+1}=0$ then $\left.v\right|_{Q} \in W^{1, p}\left(Q ; \mathbb{R}^{n}\right)$ for $p=p(\beta)$. Hence, by Lemma 3.1.1 and Theorem 3.1.8 in [28], if $a_{m+1} \geq 0$, we may infer the existence of a representative $\hat{v}$ of $v$ such that, for each $i=$ $1, \ldots, m+1, \hat{v}\left(x_{1}, \ldots, x_{i-1}, x_{i}, x_{i+1}, \ldots, x_{m+1}\right)$ is an absolutely continuous function of $x_{i}$ for almost all fixed values of $x_{1}, \ldots, x_{i-1}, x_{i+1}, \ldots, x_{m+1}$ with respect to the $m$ dimensional Hausdorff measure $\mathcal{H}^{m}$ on $\left[a_{1}, b_{1}\right] \times \cdots \times\left[a_{i-1}, b_{i-1}\right] \times\left[a_{i+1}, b_{i+1}\right] \times \cdots \times\left[a_{m+1}, b_{m+1}\right]$. The classical partial derivatives $\frac{\partial \hat{v}}{\partial x_{i}}$ agree almost everywhere with the weak derivatives $\frac{\partial v}{\partial x_{i}}$. Furthermore, for any closed subset $N$ of $\mathbb{R}^{n}$, if $v(x) \in N$ for almost every $x$ then it is possible to choose $\hat{v}(x) \in N$ for every $x \in \mathbb{R}_{+}^{m+1}$.

\subsection{Proof of Lemma 4.16}

Proof of Lemma 4.16 We follow the proof, given in Section 2.12.2 of [39], of Lemma 1 in Section 2.6 of [39]. Throughout, $C$ denotes a constant only depending on $m$ and $\beta$.

Suppose $u, v \in W_{\beta}^{1,2}\left(\mathbb{S}_{+}^{m} ; N\right)$. We reflect $u$ and $v$ evenly in $\partial \mathbb{R}_{+}^{m+1}$, without relabelling, to get $u, v \in W_{\beta}^{1,2}\left(\mathbb{S}^{m} ; N\right)$ and choose extensions of $u$ and $v$ to $\mathbb{R}^{m+1} \backslash\{0\}$ which are homogeneous of degree zero with respect to the origin. Then we choose representatives of these extensions which satisfy the absolute continuity properties described in Sect. 4.6 on $\overline{Q_{1}(0)}$. We will denote the representatives of the extensions of $u$ and $v$ by $\hat{u}$ and $\hat{v}$ respectively. Then $\hat{u}(\rho \omega)=\hat{u}(\omega), \hat{v}(\rho \omega)=\hat{v}(\omega)$ for almost every $\rho>0$ and $\omega \in \mathbb{S}^{m}$. Moreover, we have the identity $\nabla \hat{u}=|x|^{-1} \nabla \hat{u}(\omega(x))=D u(\omega(x))$ for $\omega(x)=|x|^{-1} x$, where $D$ is the gradient on $\mathbb{S}_{+}^{m}$ and $\nabla$ is the gradient on $\mathbb{R}_{+}^{m+1}$. We therefore calculate

$$
\int_{Q_{1}^{+}(0)} x_{m+1}^{\beta}\left(|\nabla \hat{u}|^{2}+|\nabla \hat{v}|^{2}\right) \mathrm{d} x \leq C \int_{\mathbb{S}_{+}^{m}} \omega_{m+1}^{\beta}\left(|D u|^{2}+|D v|^{2}\right) \mathrm{d} \omega
$$


and

$$
\int_{Q_{1}^{+}(0)} x_{m+1}^{\beta}|\hat{u}-\hat{v}|^{2} \mathrm{~d} x \leq C \int_{\mathbb{S}_{+}^{m}} \omega_{m+1}^{\beta}|u-v|^{2} \mathrm{~d} \omega .
$$

Let $\varepsilon \in\left(0, \frac{1}{8}\right)$ and define the closed rectangles $Q_{i, \varepsilon}=\left[i_{1} \varepsilon,\left(i_{1}+1\right) \varepsilon\right] \times \cdots \times$ $\left[i_{m+1} \varepsilon,\left(i_{m+1}+1\right) \varepsilon\right]$ for $i=\left(i_{1}, \ldots, i_{m+1}\right) \in \mathbb{Z}^{m+1}$. Fix $\varepsilon \in\left(0, \frac{1}{8}\right)$ arbitrarily henceforth. Let $F^{l}$ denote any $l$-dimensional face of a $Q_{i, \varepsilon}$. We define

$$
\mathcal{Q}=\left\{Q_{i, \varepsilon}: i \in \mathbb{Z}^{m+1}, Q_{i, \varepsilon} \subset \overline{Q_{\frac{1}{2}}(0)}\right\} \text { and } \mathcal{F}_{i}^{l}=\left\{F^{l} \text { faces of } Q_{i, \varepsilon}\right\} .
$$

In addition, we write $x+\mathcal{F}_{i}^{l}$ to denote the collection of the translations of all faces in $\mathcal{F}_{i}^{l}$ by $x \in \mathbb{R}^{m+1}$.

Consider a non-negative, measurable function $f: \overline{Q_{1}(0)} \rightarrow \mathbb{R}$ which is even with respect to the hyperplane $\partial \mathbb{R}_{+}^{m+1}$. Invoking [39, Section 2.12.2] estimate (3), which is a consequence of Chebychev's inequality and Fubini's theorem, we see that for every $K \geq 1$ there exists a set $P \subset Q_{0, \varepsilon}$ of measure $|P| \leq \frac{C \varepsilon^{m+1}}{K}$, with $C=C(m)$, such that for all $y \in Q_{0, \varepsilon} \backslash P$ and $l \in\{0, \ldots, m+1\}$ we have

$$
\varepsilon^{m+1-l} \sum_{\left\{i: Q_{i, \varepsilon} \in \mathcal{Q}\right\}} \sum_{y+\mathcal{F}_{i}^{l}} \int_{F^{l}} f \mathrm{~d} \mathcal{H}^{l} \leq K \int_{Q_{1}(0)} f \mathrm{~d} x=2 K \int_{Q_{1}^{+}(0)} f \mathrm{~d} x .
$$

Since we chose $\hat{u}$ and $\hat{v}$ with the absolute continuity properties described in Sect. 4.6 on $\overline{Q_{1}(0)}$ it follows that for almost every $x \in Q_{0, \varepsilon}$, with respect to the $m+1$-dimensional Lebesgue measure, all of the functions $\hat{u}, \hat{v}, \nabla \hat{u}, \nabla \hat{v}$ are $\mathcal{H}^{l}$ almost everywhere defined on each of the $l$-dimensional faces of $x+Q_{i, \varepsilon}$ for $Q_{i, \varepsilon} \in \mathcal{Q}$ and $l=1, \ldots, m+1$. Moreover, the gradients of $\hat{u}$ and $\hat{v}$ on any $l$-dimensional face of $x+Q_{i, \varepsilon}$ coincide $\mathcal{H}^{l}$ almost everywhere with the tangential parts of $\nabla \hat{u}$ and $\nabla \hat{v}$ respectively. Thus we may choose $x=a \in Q_{0, \varepsilon}$ such that these properties hold and, provided we choose $K$ (depending on $m$ ) sufficiently large in (4.24), such that $a_{m+1} \geq \frac{\varepsilon}{2}$ and such that we may apply (4.24) simultaneously for $f(x)=\left|x_{m+1}\right|^{\beta} \tilde{f}(x)$ with $\tilde{f}(x)=|\hat{u}(x)-\hat{v}(x)|^{2}$ and $\tilde{f}(x)=|\nabla \hat{u}(x)|^{2}+|\nabla \hat{v}(x)|^{2}$ (where $\nabla$ is the gradient on $\mathbb{R}^{m+1}$ ). In particular, after discarding the integrals in (4.24) taken over any cube faces which do not intersect $\mathbb{R}_{+}^{m+1}$, we have

$$
\varepsilon^{m+1-l} \sum_{\left\{i: \begin{array}{c}
Q_{i, \varepsilon} \in \mathcal{Q} \\
i_{m+1} \geq-1
\end{array}\right\}} \sum_{a+\mathcal{F}_{i}^{l}} \int_{F^{l} \cap \mathbb{R}_{+}^{m+1}} x_{m+1}^{\beta} \tilde{f} \mathrm{~d} \mathcal{H}^{l} \leq C \int_{Q_{1}^{+}(0)} x_{m+1}^{\beta} \tilde{f} \mathrm{~d} x .
$$

Now we begin the construction of $w$ by defining a map on the one dimensional faces of every $Q \times[0, \varepsilon]$ where $Q:=\left(a+Q_{i, \varepsilon}\right) \cap \mathbb{R}_{+}^{m+1}$ with $Q_{i, \varepsilon} \in \mathcal{Q}$ and $i_{m+1} \geq-1$. Let $E_{j}$ denote a one dimensional face of $Q$ parallel to the $j$ th coordinate axis for $j=1, \ldots, m+1$. Define $w(x, 0)=\hat{u}(x)$ on $Q \times\{0\}$ and $w(x, \varepsilon)=\hat{v}(x)$ on $Q \times\{\varepsilon\}$ and let $w(x, s)=$ $\left(1-\frac{s}{\varepsilon}\right) \hat{u}(x)+\frac{s}{\varepsilon} \hat{v}(x)$ for $x \in E_{j}$ and $s \in[0, \varepsilon]$. Since $\hat{u}\left(\mathbb{R}_{+}^{m+1}\right) \subset N$ by definition, it follows that

$$
\operatorname{dist}^{2}(w(x, s), N) \leq \max _{j=1, \ldots, m+1} \sup _{E_{j}}|\hat{u}-\hat{v}|^{2}
$$

for $x$ in the 1-dimensional edges of $Q$ and $s \in[0, \varepsilon]$.

We now estimate $\sup _{E_{j}}|\hat{u}-\hat{v}|^{2}$ using the Sobolev embedding theorem for $W_{\beta}^{1,2}$ along the line segments $E_{j}$, which one can deduce analogously to the case for $W^{1,2}$ functions. 
Note that in our construction so far, we have discarded any edges $E_{j} \subset \partial \mathbb{R}_{+}^{m+1}$. If $E_{j}$, $j=1, \ldots, m+1$, lies in a $Q=\left(a+Q_{i, \varepsilon}\right) \cap \mathbb{R}_{+}^{m+1}$ with $i_{m+1} \geq-1$, with the exception of the case $i_{m+1}=-1, j=m+1$ and $\beta \in(0,1)$, we calculate

$$
\begin{aligned}
\sup _{E_{j}}|\hat{u}-\hat{v}|^{2} \leq & \frac{C}{\varepsilon^{\frac{\beta}{2}+\frac{|\beta|}{2}}}\left(\int_{E_{j}} x_{m+1}^{\beta}\left(\left|\partial_{j} \hat{u}\right|^{2}+\left|\partial_{j} \hat{v}\right|^{2}\right) \mathrm{d} x_{j}\right)^{\frac{1}{2}}\left(\int_{E_{j}} x_{m+1}^{\beta}|\hat{u}-\hat{v}|^{2} \mathrm{~d} x_{j}\right)^{\frac{1}{2}} \\
& +\frac{C}{\varepsilon^{1+\frac{\beta}{2}+\frac{|\beta|}{2}}} \int_{E_{j}} x_{m+1}^{\beta}|\hat{u}-\hat{v}|^{2} \mathrm{~d} x_{j} .
\end{aligned}
$$

If $\beta>0, i_{m+1}=-1$ and $j=m+1$ then we calculate

$$
\begin{aligned}
& \sup _{E_{m+1}}|\hat{u}-\hat{v}|^{2} \\
& \quad \leq C\left(\int_{E_{m+1}} x_{m+1}^{\beta}\left(\left|\partial_{m+1} \hat{u}\right|^{2}+\left|\partial_{m+1} \hat{v}\right|^{2}\right) \mathrm{d} x_{m+1}\right)^{\frac{1}{p}}\left(\int_{E_{m+1}} x_{m+1}^{\beta}|\hat{u}-\hat{v}|^{2} \mathrm{~d} x_{m+1}\right)^{1-\frac{1}{p}} \\
& \quad+C \varepsilon^{-(1+\beta)} \int_{E_{m+1}} x_{m+1}^{\beta}|\hat{u}-\hat{v}|^{2} \mathrm{~d} x_{m+1}
\end{aligned}
$$

for any $p \in\left(1, \frac{2}{1+\beta}\right)$. The combination of (4.26), (4.27) and (4.28) with (4.25), applied with $l=1$, yields

$$
\begin{aligned}
\operatorname{dist}^{2}(w(x, s), N) \leq & \frac{C}{\varepsilon^{m+\frac{\beta}{2}+\frac{|\beta|}{2}}}\left(\int_{Q_{1}^{+}(0)} x_{m+1}^{\beta}\left(|\nabla \hat{u}|^{2}+|\nabla \hat{v}|^{2}\right) \mathrm{d} x\right)^{\frac{1}{q}} \\
& \left(\int_{Q_{1}^{+}(0)} x_{m+1}^{\beta}|\hat{u}-\hat{v}|^{2} \mathrm{~d} x\right)^{1-\frac{1}{q}} \\
& +\frac{C}{\varepsilon^{m+1+\frac{\beta}{2}+\frac{|\beta|}{2}}} \int_{Q_{1}^{+}(0)} x_{m+1}^{\beta}|\hat{u}-\hat{v}|^{2} \mathrm{~d} x
\end{aligned}
$$

where $q \in\{p, 2\}$, for $p$ fixed as above, depends on $\beta$.

Next we bound the gradient of $w$ on the product of the 1-dimensional edges of $Q$ with $[0, \varepsilon]$. Let $\bar{\nabla}$ denote the gradient on $E_{j} \times[0, \varepsilon]$. Recall that $\hat{u}, \hat{v}$ are defined so that the tangential parts of their gradients $\nabla \hat{u}, \nabla \hat{v}$ on $\mathbb{R}_{+}^{m+1}$ coincide $\mathcal{H}^{1}$ almost everywhere with their gradients on the edges $E_{j}$. It follows that

$$
\sup _{s \in[0, \varepsilon]}|\bar{\nabla} w(x, s)|^{2} \leq 8\left(|\nabla \hat{u}(x)|^{2}+|\nabla \hat{v}(x)|^{2}\right)+\frac{2}{\varepsilon^{2}}|\hat{u}(x)-\hat{v}(x)|^{2},
$$

for $x$ in any edge $E_{j}, j=1, \ldots, m+1$, of $Q$. Integrating over $E_{j} \times[0, \varepsilon]$ with respect to $x_{m+1}^{\beta} \mathrm{d} x_{j} \mathrm{~d} s$ yields

$$
\int_{E_{j} \times[0, \varepsilon]} x_{m+1}^{\beta}|\bar{\nabla} w|^{2} \mathrm{~d} x_{j} \mathrm{~d} s \leq 8 \varepsilon \int_{E_{j}} x_{m+1}^{\beta}\left(|\nabla \hat{u}|^{2}+|\nabla \hat{v}|^{2}\right) \mathrm{d} x_{j}+\frac{2}{\varepsilon} \int_{E_{j}} x_{m+1}^{\beta}|\hat{u}-\hat{v}|^{2} \mathrm{~d} x_{j} .
$$

Consider again $Q \times[0, \varepsilon]$ for $Q=\left(a+Q_{i, \varepsilon}\right) \cap \mathbb{R}_{+}^{m+1}$ with $Q_{i, \varepsilon} \in \mathcal{Q}$ and $i_{m+1} \geq-1$. Recall that we are excluding cube faces in $\partial \mathbb{R}_{+}^{m+1}$ from our construction. We use a slightly different procedure to extend $w$ to higher dimensions depending on whether $i_{m+1}=-1$ or $i_{m+1} \geq 0$. Accordingly we introduce some temporary notation for two classes of $F^{l}$ that we 
consider. Let $F_{\perp}^{l}$ denote any $l$-dimensional face of any $Q$ with no edges in the $m+1$ direction and let $F_{m+1}^{l}$ denote any face of any such $Q$ with edges in the $m+1$ direction. Suppose that $l \geq 2$ and $w$ is already defined with $L^{2}$ gradient on every $F_{\perp}^{l}$ and $F_{\perp}^{l-1} \times[0, \varepsilon]$, square $x_{m+1}^{\beta} \mathrm{d} \mathcal{H}^{l}$-integrable gradient on every $F_{m+1}^{l}$ and square $x_{m+1}^{\beta} \mathrm{d} \mathcal{H}^{l-1} \mathrm{~d} s$-integrable gradient on $F_{m+1}^{l-1} \times[0, \varepsilon]$. Furthermore, suppose that $w(x, 0)=\hat{u}(x)$ and $w(x, \varepsilon)=\hat{v}(x)$ for $x \in F_{\perp}^{l}$ or $x \in F_{m+1}^{l}$. These assumptions imply that $w$ is defined $\mathcal{H}^{l}$ almost everywhere on all the $l$-dimensional faces of $Q$ for $l \geq 2$. Since $\partial\left(F_{\perp}^{l} \times[0, \varepsilon]\right)$ and $\partial^{+}\left(F_{m+1}^{l} \times[0, \varepsilon]\right)$ are the union of such $l$-dimensional faces, $w$ is defined $\mathcal{H}^{l}$ almost everywhere on these sets. If $Q$ is such that $i_{m+1} \geq 0$ then we do not distinguish between $F_{\perp}^{l}$ and $F_{m+1}^{l}$ and extend $w$ to each $F_{\perp}^{l} \times[0, \varepsilon]$ and $F_{m+1}^{l} \times[0, \varepsilon]$ by homogeneous extension of degree zero with respect to $\left(y, \frac{\varepsilon}{2}\right)$, where $y$ is the centre point of $F_{\perp}^{l}$ or $F_{m+1}^{l}$. If $i_{m+1}=-1$ then we extend $w$ into $F_{\perp}^{l} \times[0, \varepsilon]$ using the same method. We extend $w$ homogeneously of degree 0 from $\partial^{+}\left(F_{m+1}^{l} \times[0, \varepsilon]\right)$ into $F_{m+1}^{l} \times[0, \varepsilon]$ with respect to the point $\left(y^{+}, \frac{\varepsilon}{2}\right)$, where $y$ is the centre point of $F_{m+1}^{l}$ and $y^{+}=y-\left(0, y_{m+1}\right)$.

Now let $F^{l}$ denote any $l$-dimensional face of any $Q$ again. Since the tangential parts of the gradients $\nabla \hat{u}, \nabla \hat{v}$ on $\mathbb{R}^{m+1}$ coincide with the gradients of $\hat{u}$ and $\hat{v}$ on $F^{l}$ for $\mathcal{H}^{l}$ almost every $x \in F^{l}$, using the fact that $\hat{u}$ and $\hat{v}$ are homogeneous of degree zero, we calculate

$$
\begin{aligned}
\int_{F^{l} \times[0, \varepsilon]} x_{m+1}^{\beta}|\bar{\nabla} w|^{2} \mathrm{~d} \mathcal{H}^{l} \mathrm{~d} s \leq & C \varepsilon \int_{F^{l}} x_{m+1}^{\beta}\left(|\nabla \hat{u}|^{2}+|\nabla \hat{v}|^{2}\right) \mathrm{d} \mathcal{H}^{l} \\
& +C \varepsilon \sum_{a+\mathcal{F}_{i}^{l-1}} \int_{F^{l-1} \times[0, \varepsilon]} x_{m+1}^{\beta}|\bar{\nabla} w|^{2} \mathrm{~d} \mathcal{H}^{l-1} \mathrm{~d} s,
\end{aligned}
$$

where $\bar{\nabla}$ is the gradient on the set it is integrated over. From (4.31), we inductively deduce that for any $l \in\{2, \ldots, m+1\}$ we can extend $w$ to each $F^{l} \times[0, \varepsilon]$ in $Q \times[0, \varepsilon]$ so that $w$ has an $L^{2}$ or $x_{m+1}^{\beta} \mathrm{d} \mathcal{H}^{l} \mathrm{~d} s$-integrable gradient $\bar{\nabla} w$ on these faces. Moreover, $\bar{\nabla} w$ satisfies

$$
\begin{aligned}
\int_{F^{l} \times[0, \varepsilon]} x_{m+1}^{\beta}|\bar{\nabla} w|^{2} \mathrm{~d} \mathcal{H}^{l} \mathrm{~d} s \leq & C \varepsilon^{l-1} \sum_{a+\mathcal{F}_{i}^{1}} \int_{F^{1} \times[0, \varepsilon]} x_{m+1}^{\beta}|\bar{\nabla} w|^{2} \mathrm{~d} \mathcal{H}^{1} \mathrm{~d} s \\
& +C \sum_{j=1}^{l} \varepsilon^{l-j+1} \sum_{a+\mathcal{F}_{i}^{j}} \int_{F^{j}} x_{m+1}^{\beta}\left(|\nabla \hat{u}|^{2}+|\nabla \hat{v}|^{2}\right) \mathrm{d} \mathcal{H}^{j}
\end{aligned}
$$

So far, we have constructed a map $w=w^{i, \varepsilon}$ on each cube and rectangle $Q=(a+$ $\left.Q_{i, \varepsilon}\right) \cap \mathbb{R}_{+}^{m+1}$ such that $Q_{i, \varepsilon} \in \mathcal{Q}$ with $i_{m+1} \geq-1$. It follows from the construction that $w^{(i, \varepsilon)}=w^{(j, \varepsilon)} \mathcal{H}^{l+1}$-almost everywhere on common faces $F^{l} \times[0, \varepsilon]$ of $\left(a+Q_{i, \varepsilon}\right) \cap \mathbb{R}_{+}^{m+1}$ and $\left(a+Q_{j, \varepsilon}\right) \cap \mathbb{R}_{+}^{m+1}$. Furthermore, for $0<\varepsilon<\frac{1}{8}$ it follows that

$$
Q_{\frac{1}{4}}^{+}(0) \subset \bigcup_{\left\{i: \begin{array}{c}
Q_{i, \varepsilon} \in \mathcal{Q} \\
i_{m+1} \geq-1
\end{array}\right\}} a+Q_{i, \varepsilon} .
$$

We may therefore define $w \in W_{\beta}^{1,2}\left(Q_{\frac{1}{4}}^{+}(0) \times[0, \varepsilon] ; \mathbb{R}^{n}\right)$ by $\left.w\right|_{\left(a+Q_{i, \varepsilon}\right) \cap \mathbb{R}_{+}^{m+1}}(x, s)=$ $w^{(i, \varepsilon)}(x, s)$ for $s \in[0, \varepsilon]$. Since $w$ is homogeneous of degree 0 on any $l$-dimensional face of any $Q \times[0, \varepsilon]$ with $l \geq 3$, our inductive procedure preserves (4.29) for all $(x, s)$ in 
$Q_{\frac{1}{4}}^{+}(0) \times[0, \varepsilon]$, with the possible exception of a set $P$ of $m$-dimensional Hausdorff measure 0 . It follows from (4.29) that for $(x, s) \in\left(Q_{\frac{1}{4}}^{+}(0) \times[0, \varepsilon]\right) \backslash P$ we have

$$
\begin{aligned}
\operatorname{dist}^{2}(w(x, s), N) \leq & \frac{C}{\varepsilon^{m+\frac{\beta}{2}+\frac{|\beta|}{2}}}\left(\int_{Q_{1}^{+}(0)} x_{m+1}^{\beta}\left(|\nabla \hat{u}|^{2}+|\nabla \hat{v}|^{2}\right) \mathrm{d} x\right)^{\frac{1}{q}} \\
& \left(\int_{Q_{1}^{+}(0)} x_{m+1}^{\beta}|\hat{u}-\hat{v}|^{2} \mathrm{~d} x\right)^{1-\frac{1}{q}} \\
& +\frac{C}{\varepsilon^{m+1+\frac{\beta}{2}+\frac{|\beta|}{2}}} \int_{Q_{1}^{+}(0)} x_{m+1}^{\beta}|\hat{u}-\hat{v}|^{2} \mathrm{~d} x
\end{aligned}
$$

where $q \in\{2, p\}$ for some fixed $p \in\left(1, \frac{2}{1+\beta}\right)$. Moreover, we combine (4.30), (4.32) and (4.25) to see that

$$
\begin{aligned}
\int_{Q_{\frac{1}{4}}^{+}(0) \times[0, \varepsilon]} x_{m+1}^{\beta}|\bar{\nabla} w|^{2} \mathrm{~d} x \mathrm{~d} s \leq & C \varepsilon \int_{Q_{1}^{+}(0)} x_{m+1}^{\beta}\left(|\nabla \hat{u}|^{2}+|\nabla \hat{v}|^{2}\right) \mathrm{d} x \\
& +\frac{C}{\varepsilon} \int_{Q_{1}^{+}(0)} x_{m+1}^{\beta}|\hat{u}-\hat{v}|^{2} \mathrm{~d} x .
\end{aligned}
$$

The definition of $w$ as required now follows from combining (4.33) and (4.34) with (4.22) and (4.23). The absolute continuity properties, described in Sect. 4.6, of $w$, viewed as a function defined on a rectangle in polar coordinates, guarantee that for almost every $\rho \in\left[\frac{1}{8}, \frac{1}{4}\right], w$ has square $x_{m+1}^{\beta} \mathrm{d} \mathcal{H}^{m} \mathrm{~d} s$-integrable gradient on $\partial^{+} B_{\rho}^{+}(0) \times[0, \varepsilon]$ which coincides $\mathcal{H}^{m} \mathrm{~d} s$ almost everywhere with the tangential part of $\bar{\nabla} w$. Using Fubini's theorem and Chebychev's inequality, applied to the map $\rho \mapsto \int_{\partial^{+} B_{\rho}^{+}(0) \times[0, \varepsilon]} x_{m+1}^{\beta}|\bar{\nabla} w|^{2} \mathrm{~d} \mathcal{H}^{m} \mathrm{~d} s$, we may therefore choose $\rho \in\left[\frac{1}{8}, \frac{1}{4}\right]$ such that $w$ has square $x_{m+1}^{\beta} \mathrm{d} \mathcal{H}^{m} \mathrm{~d} s$-integrable gradient on $\partial^{+} B_{\rho}^{+}(0) \times[0, \varepsilon]$ and satisfies

$$
\int_{\partial^{+} B_{\rho}^{+}(0) \times[0, \varepsilon]} x_{m+1}^{\beta}|\bar{\nabla} w|^{2} \mathrm{~d} \mathcal{H}^{m} \mathrm{~d} s \leq C \int_{B_{\frac{1}{4}}^{+}(0) \times[0, \varepsilon]} x_{m+1}^{\beta}|\bar{\nabla} w|^{2} \mathrm{~d} \mathcal{H}^{m+1} \mathrm{~d} s .
$$

We define $\tilde{w}$ on $\mathbb{S}_{+}^{m} \times[0, \varepsilon]$ by $\tilde{w}(\omega, s)=w(\rho \omega, s)$ and observe that this map has the required properties.

\subsection{Comparison maps}

With Lemma 4.16 in hand, we may now construct comparison maps for $W_{\beta}^{1,2}$ functions which have values in $N$ and are defined on half-balls centred in $\partial \mathbb{R}_{+}^{m+1}$, provided the re-scaled energy is sufficiently small. We use the notation $\bar{v}_{B_{\rho}^{+}(y), \beta}=\left(\int_{B_{\rho}^{+}(y)} x_{m+1}^{\beta} \mathrm{d} x\right)^{-1} \int_{B_{\rho}^{+}(y)} x_{m+1}^{\beta} v \mathrm{~d} x$.

Lemma 4.17 There exists a $\delta_{0}=\delta_{0}(m, N, \beta)>0$ such that the following holds. Let $\varepsilon \in$ $(0,1)$ and $v \in W_{\beta}^{1,2}\left(B_{\rho}^{+}(y) ; N\right)$ with $\rho^{1-m-\beta} \int_{B_{\rho}^{+}(y)} x_{m+1}^{\beta}|\nabla v|^{2} \mathrm{~d} x \leq \delta_{0}^{2} \varepsilon^{m+1+\frac{\beta}{2}+\frac{|\beta|}{2}}$, where $y \in \partial \mathbb{R}_{+}^{m+1}$. Then there is a $\sigma \in\left(\frac{3 \rho}{4}, \rho\right)$ such that we can find a map $w_{\varepsilon} \in W_{\beta}^{1,2}\left(B_{\rho}^{+}(y) ; N\right)$ which agrees with $v$ in $B_{\rho}^{+}(y) \backslash B_{\sigma}^{+}(y)$ and such that

$$
\sigma^{1-m-\beta} \int_{B_{\sigma}^{+}(y)} x_{m+1}^{\beta}|\nabla w|^{2} \mathrm{~d} x \leq C \varepsilon \rho^{1-m-\beta} \int_{B_{\rho}^{+}(y)} x_{m+1}^{\beta}|\nabla v|^{2} \mathrm{~d} x
$$




$$
+\frac{C}{\varepsilon} \rho^{-(1+m+\beta)} \int_{B_{\rho}^{+}(y)} x_{m+1}^{\beta}\left|v-\bar{v}_{B_{\rho}^{+}(y), \beta}\right|^{2} \mathrm{~d} x
$$

for a constant $C=C(m, \beta)$.

Proof We follow the proof of Corollary 1 in Section 2.7 of [39]. Throughout, $C$ denotes a constant which depends on $m$ and possibly $\beta$ and we only distinguish different $C$ when necessary. We also assume, without loss of generality, that $\varepsilon \leq \frac{1}{2}$.

Let $\delta_{0}>0$ to be chosen as required and suppose the assumptions of the lemma hold for $\delta_{0}$. As a consequence of Poincaré Inequality for the $A_{2}$ weights $\left|x_{m+1}\right|^{\beta}$ [19], we have

$$
\begin{aligned}
& \rho^{-(1+m+\beta)} \int_{B_{\rho}^{+}(y)} x_{m+1}^{\beta}\left|v-\bar{v}_{B_{\rho}^{+}(y), \beta}\right|^{2} \mathrm{~d} x \\
& \leq C \rho^{1-m-\beta} \int_{B_{\rho}^{+}(y)} x_{m+1}^{\beta}|\nabla v|^{2} \mathrm{~d} x \leq C \delta_{0}^{2} \varepsilon^{m+1+\frac{\beta}{2}+\frac{|\beta|}{2}} .
\end{aligned}
$$

As discussed in Sect. 4.6, we may work with a representative of $v$, which we do not relabel, such that $v\left(B_{\rho}^{+}(y)\right) \subset N$. It follows that

$$
\operatorname{dist}^{2}\left(\bar{v}_{B_{\rho}^{+}(y), \beta}, N\right) \leq\left|v(x)-\bar{v}_{B_{\rho}^{+}(y), \beta}\right|^{2}
$$

for every $x \in B_{\rho}^{+}(y)$. Integrating (4.37) over $B_{\rho}^{+}(y)$ with respect to $x_{m+1}^{\frac{\beta}{2}+\frac{|\beta|}{2}} \mathrm{~d} x$ and then dividing by $\int_{B_{\rho}^{+}(y)} x_{m+1}^{\frac{\beta}{2}+\frac{|\beta|}{2}} \mathrm{~d} x$ we see that

$$
\operatorname{dist}^{2}\left(\bar{v}_{B_{\rho}^{+}(y), \beta}, N\right) \leq C \rho^{-(1+m+\beta)} \int_{B_{\rho}^{+}(y)} x_{m+1}^{\beta}\left|v-\bar{v}_{B_{\rho}^{+}(y), \beta}\right|^{2} \mathrm{~d} x .
$$

Combining (4.38) with (4.36) we find

$$
\operatorname{dist}^{2}\left(\bar{v}_{B_{\rho}^{+}(y), \beta}, N\right) \leq C \rho^{-(1+m+\beta)} \int_{B_{\rho}^{+}(y)} x_{m+1}^{\beta}\left|v-\bar{v}_{B_{\rho}^{+}(y), \beta}\right|^{2} \mathrm{~d} x \leq C \delta_{0}^{2} \varepsilon^{m+1+\frac{\beta}{2}+\frac{|\beta|}{2}} .
$$

Hence, we may choose $\lambda \in N$ such that

$$
\begin{aligned}
\left|\lambda-\bar{v}_{B_{\rho}^{+}(y), \beta}\right|^{2} & \leq C \rho^{-(1+m+\beta)} \int_{B_{\rho}^{+}(y)} x_{m+1}^{\beta}\left|v-\bar{v}_{B_{\rho}^{+}(y), \beta}\right|^{2} \mathrm{~d} x \\
& \leq C \delta_{0}^{2} \varepsilon^{m+1+\frac{\beta}{2}+\frac{|\beta|}{2}} .
\end{aligned}
$$

Using Chebychev's inequality, we choose a $C>0$ such that there exists $\sigma \in\left(\frac{3 \rho}{4}, \rho\right)$ such that $\hat{v} \in W_{\beta}^{1,2}\left(\mathbb{S}_{+}^{m} ; N\right)$, where $\hat{v}(\omega)=v(\sigma \omega+y)$, and such that

$$
\begin{aligned}
\int_{\mathbb{S}_{+}^{m}} \omega_{m+1}^{\beta}|D \hat{v}|^{2} \mathrm{~d} \omega & \leq C \sigma^{2-m-\beta} \int_{\partial^{+} B_{\sigma}^{+}(y)} x_{m+1}^{\beta}|\nabla v|^{2} \mathrm{~d} S(x) \\
& \leq C \rho^{1-m-\beta} \int_{B_{\rho}^{+}(y) \backslash B_{\frac{\rho}{2}}^{+}(y)} x_{m+1}^{\beta}|\nabla v|^{2} \mathrm{~d} x \\
& \leq C \delta_{0}^{2} \varepsilon^{m+1+\frac{\beta}{2}+\frac{|\beta|}{2}}
\end{aligned}
$$

where $D$ is the gradient on $\mathbb{S}_{+}^{m}$, and

$$
\int_{\mathbb{S}_{+}^{m}} \omega_{m+1}^{\beta}\left|\hat{v}-\bar{v}_{B_{\rho}^{+}(y), \beta}\right|^{2} \mathrm{~d} \omega \leq \sigma^{-m-\beta} \int_{\partial^{+} B_{\sigma}^{+}(y)} x_{m+1}^{\beta}\left|v-\bar{v}_{B_{\rho}^{+}(y), \beta}\right|^{2} \mathrm{~d} S(x)
$$




$$
\begin{aligned}
& \leq C \rho^{-(1+m+\beta)} \int_{B_{\rho}^{+}(y) \backslash B_{\frac{\rho}{2}}^{+}(y)} x_{m+1}^{\beta}\left|v-\bar{v}_{B_{\rho}^{+}(y), \beta}\right|^{2} \mathrm{~d} x \\
& \leq C \delta_{0}^{2} \varepsilon^{m+1+\frac{\beta}{2}+\frac{|\beta|}{2}} .
\end{aligned}
$$

We may therefore apply Lemma 4.16 to $\hat{v} \in W_{\beta}^{1,2}\left(\mathbb{S}_{+}^{m} ; N\right)$ and $\lambda$. This yields a $w_{0}: \mathbb{S}_{+}^{m} \times$ $[0, \varepsilon] \rightarrow \mathbb{R}^{n}$ with $w_{0}=\hat{v}$ on $\mathbb{S}_{+}^{m} \times\{0\}$ and $w_{0}=\lambda$ on $\mathbb{S}_{+}^{m} \times\{\varepsilon\}$ in the sense of traces. Furthermore, (4.20) yields

$$
\begin{aligned}
\int_{\mathbb{S}_{+}^{m} \times[0, \varepsilon]} \omega_{m+1}^{\beta}\left|\bar{D} w_{0}\right|^{2} \mathrm{~d} \omega \mathrm{d} s \leq & C \varepsilon \int_{\mathbb{S}_{+}^{m}} \omega_{m+1}^{\beta}|D \hat{v}|^{2} \mathrm{~d} \omega \\
& +\frac{C}{\varepsilon} \int_{\mathbb{S}_{+}^{m}} \omega_{m+1}^{\beta}|\hat{v}-\lambda|^{2} \mathrm{~d} \omega,
\end{aligned}
$$

where $\bar{D}$ is the gradient on $\mathbb{S}_{+}^{m} \times[0, \varepsilon]$ and $D$ is the gradient on $\mathbb{S}_{+}^{m}$. In addition, (4.21) implies that

$$
\begin{aligned}
\operatorname{dist}^{2}\left(w_{0}(\omega, s), N\right) \leq & \frac{C}{\varepsilon^{m+\frac{\beta}{2}+\frac{|\beta|}{2}}}\left(\int_{\mathbb{S}_{+}^{m}} \omega_{m+1}^{\beta}|D \hat{v}|^{2} \mathrm{~d} \omega\right)^{\frac{1}{q}}\left(\int_{\mathbb{S}_{+}^{m}} \omega_{m+1}^{\beta}|\hat{v}-\lambda|^{2} \mathrm{~d} \omega\right)^{1-\frac{1}{q}} \\
& +\frac{C}{\varepsilon^{m+1+\frac{\beta}{2}+\frac{|\beta|}{2}}} \int_{\mathbb{S}_{+}^{m}} \omega_{m+1}^{\beta}|\hat{v}-\lambda|^{2} \mathrm{~d} \omega
\end{aligned}
$$

for every $(\omega, s) \in \mathbb{S}_{+}^{m} \times[0, \varepsilon]$, where $q \in(1,2]$ depends on $\beta$. Henceforth we assume that $\delta_{0} \leq 1$. Using (4.39) and (4.41) we deduce that

$$
\begin{aligned}
\int_{\mathbb{S}_{+}^{m}} \omega_{m+1}^{\beta}|\hat{v}-\lambda|^{2} \mathrm{~d} \omega & \leq 2 \int_{\mathbb{S}_{+}^{m}} \omega_{m+1}^{\beta}\left|\hat{v}-\bar{v}_{B_{\rho}^{+}(y), \beta}\right|^{2} \mathrm{~d} \omega+2 \int_{\mathbb{S}_{+}^{m}} \omega_{m+1}^{\beta}\left|\bar{v}_{B_{\rho}^{+}(y), \beta}-\lambda\right|^{2} \mathrm{~d} \omega \\
& \leq C \delta_{0}^{2} \varepsilon^{m+1+\frac{\beta}{2}+\frac{|\beta|}{2}} .
\end{aligned}
$$

The combination of (4.43) with (4.40) and (4.44) yields

$$
\operatorname{dist}\left(w_{0}(x, s), N\right) \leq C \delta_{0}
$$

for every $(\omega, s) \in \mathbb{S}_{+}^{m} \times[0, \varepsilon]$.

Choose $\delta_{0}$, depending on $N, m, \beta$, such that $C \delta_{0} \leq \hat{\alpha}$ where $C$ is the constant in (4.45) and $\hat{\alpha}>0$ is sufficiently small to guarantee that the nearest point projection $\pi_{N}$ onto $N$ exists and has bounded derivatives in $N_{\hat{\alpha}}=\left\{x \in \mathbb{R}^{n}: \operatorname{dist}(x, N) \leq \hat{\alpha}\right\}$. It then follows from (4.45) that we may apply $\pi_{N}$ to $w_{0}$. Let $\omega \in \mathbb{S}_{+}^{m}$ satisfy $\omega=\omega(x)=\frac{x-y}{|x-y|}, r=|x-y|$ and define $w \in W_{\beta}^{1,2}\left(B_{\rho}^{+}(y) ; N\right)$ by

$$
w(x)=w(y+r \omega(x))= \begin{cases}v(y+r \omega(x)) & r \in(\sigma, \rho) \\ \pi_{N}\left(w_{0}\left(\omega(x),\left(1-\frac{r}{\sigma}\right)\right)\right) & r \in[(1-\varepsilon) \sigma, \sigma] \\ \lambda & r \in(0,(1-\varepsilon) \sigma) .\end{cases}
$$

Note that $w$ agrees with $v$ in $B_{\rho}^{+}(y) \backslash B_{\sigma}^{+}(y)$. We then readily calculate that $w$ satisfies (4.35) as required.

\subsection{Control of the mean squared oscillation}

The Euler-Lagrange equations of $E^{\beta}$ satisfy the structural conditions $\left|\operatorname{div}\left(x_{m+1}^{\beta} \nabla v\right)\right| \leq$ $c_{0} x_{m+1}^{\beta}|\nabla v|^{2}$, together with the Neumann condition (4.2). For functions satisfying such 
conditions, if the re-scaled energy is sufficiently small it is possible to control their mean squared oscillation using the energy as follows.

Lemma 4.18 For every $\delta>0$ and every $c_{0}>0$ there exist two constants $\varepsilon=$ $\varepsilon\left(m, n, \delta, c_{0}\right)>0$ and $\theta=\theta\left(m, n, \delta, c_{0}\right) \in\left(0, \frac{1}{4}\right]$ such that the following holds. Let $x_{0} \in \partial \mathbb{R}_{+}^{m+1}, R>0$ and $B_{R}^{+}\left(x_{0}\right) \subset \mathbb{R}_{+}^{m+1}$. Suppose $v \in W_{\beta}^{1,2}\left(B_{R}^{+}\left(x_{0}\right) ; \mathbb{R}^{n}\right)$ satisfies

$$
\left|\int_{B_{R}^{+}\left(x_{0}\right)} x_{m+1}^{\beta}\langle\nabla v, \nabla \phi\rangle \mathrm{d} x\right| \leq c_{0} \int_{B_{R}^{+}\left(x_{0}\right)} x_{m+1}^{\beta}|\phi||\nabla v|^{2} \mathrm{~d} x
$$

for every $\phi \in C_{0}^{\infty}\left(B_{R}\left(x_{0}\right) ; \mathbb{R}^{n}\right)$. If

$$
R^{1-m-\beta} \int_{B_{R}^{+}\left(x_{0}\right)} x_{m+1}^{\beta}|\nabla v|^{2} \mathrm{~d} x \leq \varepsilon
$$

then

$$
(\theta R)^{-(1+m+\beta)} \int_{B_{\theta R}^{+}\left(x_{0}\right)} x_{m+1}^{\beta}\left|v-\bar{v}_{B_{\theta R}^{+}\left(x_{0}\right), \beta}\right|^{2} \mathrm{~d} x \leq \delta R^{1-m-\beta} \int_{B_{R}^{+}\left(x_{0}\right)} x_{m+1}^{\beta}|\nabla v|^{2} \mathrm{~d} x .
$$

Proof The proof of the lemma is based on a blow-up procedure analogous to that of the proof of Lemma 3.5 in [29] for example.

Observe that the statement of the lemma is invariant under rescaling and translation by any point in $\partial \mathbb{R}_{+}^{m+1}$; henceforth we assume $R=1$ and $x_{0}=0$. Suppose, for a contradiction, that there exist $\delta>0$ and $c_{0}>0$ such that the claim is false. Then for any $\theta \in\left(0, \frac{1}{4}\right]$ there is a sequence of maps $\left(v_{k}\right)_{k \in \mathbb{N}}$, with $v_{k} \in W_{\beta}^{1,2}\left(B_{1}^{+}(0) ; \mathbb{R}^{n}\right)$ for every $k$, such that

$$
\left|\int_{B_{1}^{+}(0)} x_{m+1}^{\beta}\left\langle\nabla v_{k}, \nabla \phi\right\rangle \mathrm{d} x\right| \leq c_{0} \int_{B_{1}^{+}(0)} x_{m+1}^{\beta}|\phi|\left|\nabla v_{k}\right|^{2} \mathrm{~d} x
$$

for every $\phi \in C_{0}^{\infty}\left(B_{1}(0) ; \mathbb{R}^{n}\right)$ and

$$
\int_{B_{1}^{+}(0)} x_{m+1}^{\beta}\left|\nabla v_{k}\right|^{2} \mathrm{~d} x:=\varepsilon_{k} \rightarrow 0 \text { as } k \rightarrow \infty
$$

but

$$
\theta^{-(1+m+\beta)} \int_{B_{\theta}^{+}(0)} x_{m+1}^{\beta}\left|v_{k}-{\overline{\left(v_{k}\right)}}_{B_{\theta}^{+}(0), \beta}\right|^{2} \mathrm{~d} x>\delta \int_{B_{1}^{+}(0)} x_{m+1}^{\beta}\left|\nabla v_{k}\right|^{2} \mathrm{~d} x=\delta \varepsilon_{k} .
$$

Consider the normalised sequence $\left(w_{k}\right)_{k \in \mathbb{N}}$ defined by $w_{k}=\varepsilon_{k}^{-\frac{1}{2}}\left(v_{k}-{\overline{\left(v_{k}\right)}}_{B_{\theta}^{+}(0), \beta}\right)$. Then $\nabla w_{k}=\varepsilon_{k}^{-\frac{1}{2}} \nabla v_{k}$ and thus

$$
\int_{B_{1}^{+}(0)} x_{m+1}^{\beta}\left|\nabla w_{k}\right|^{2} \mathrm{~d} x=1 \text { and }{\overline{\left(w_{k}\right)}}_{B_{\theta}^{+}(0), \beta}=0 .
$$

Furthermore, we deduce from (4.47) that

$$
\theta^{-(1+m+\beta)} \int_{B_{\theta}^{+}(0)} x_{m+1}^{\beta}\left|w_{k}\right|^{2} \mathrm{~d} x>\delta .
$$

Using (4.48) and the Poincare inequality for $A_{2}$ weights, we deduce that the sequence $\left(w_{k}\right)_{k \in \mathbb{N}}$ is bounded in $W_{\beta}^{1,2}\left(B_{1}^{+}(0) ; \mathbb{R}^{n}\right)$. Hence, the Compactness Lemma, Lemma 2.5, 
yields a subsequence $\left(w_{k_{j}}\right)_{j \in \mathbb{N}}$ which converges weakly in $W_{\beta}^{1,2}\left(B_{1}^{+}(0) ; \mathbb{R}^{n}\right)$ and strongly in $L_{\beta}^{2}\left(B_{1}^{+}(0) ; \mathbb{R}^{n}\right)$ to a $w \in W_{\beta}^{1,2}\left(B_{1}^{+}(0) ; \mathbb{R}^{n}\right)$.

In view of (4.46) and (4.48) we calculate

$$
\left|\int_{B_{1}^{+}(0)} x_{m+1}^{\beta}\left\langle\nabla w_{k}, \nabla \phi\right\rangle \mathrm{d} x\right| \leq c_{0}\|\phi\|_{L^{\infty}\left(B_{1}^{+}(0) ; \mathbb{R}^{n}\right)} \varepsilon_{k}^{\frac{1}{2}}
$$

for every $\phi \in C_{0}^{\infty}\left(B_{1}(0) ; \mathbb{R}^{n}\right)$. Since $w_{k_{j}} \rightarrow w$ in $W_{\beta}^{1,2}\left(B_{1}^{+}(0) ; \mathbb{R}^{n}\right)$, it follows that

$$
\begin{aligned}
\left|\int_{B_{1}^{+}(0)} x_{m+1}^{\beta}\langle\nabla w, \nabla \phi\rangle \mathrm{d} x\right| & =\lim _{j \rightarrow \infty}\left|\int_{B_{1}^{+}(0)} x_{m+1}^{\beta}\left\langle\nabla w_{k_{j}}, \nabla \phi\right\rangle \mathrm{d} x\right| \\
& \leq c_{0}\|\phi\|_{L^{\infty}\left(B_{1}^{+}(0) ; \mathbb{R}^{n}\right)} \lim _{j \rightarrow \infty} \varepsilon_{k_{j}}^{\frac{1}{2}}=0
\end{aligned}
$$

for every $\phi \in C_{0}^{\infty}\left(B_{1}(0) ; \mathbb{R}^{n}\right)$. Hence $w$ is a weak solution of the linear Neumann-type problem (2.11) and, in particular, satisfies (2.12) from Lemma 2.7 in $B_{1}^{+}(0)$.

We also conclude, using the Compactness Lemma to take limits in (4.48) and (4.49), that

$$
\int_{B_{1}^{+}(0)} x_{m+1}^{\beta}|\nabla w|^{2} \mathrm{~d} x \leq 1 \text { and } \bar{w}_{B_{\theta}^{+}(0), \beta}=0
$$

and

$$
\theta^{-(1+m+\beta)} \int_{B_{\theta}^{+}(0)} x_{m+1}^{\beta}|w|^{2} \mathrm{~d} x \geq \delta
$$

respectively. Now, since $\bar{w}_{B_{\theta}^{+}(0), \beta}=0$, the Poincaré inequality yields

$$
\theta^{-(1+m+\beta)} \int_{B_{\theta}^{+}(0)} x_{m+1}^{\beta}|w|^{2} \mathrm{~d} x \leq C \theta^{1-m-\beta} \int_{B_{\theta}^{+}(0)} x_{m+1}^{\beta}|\nabla w|^{2} \mathrm{~d} x .
$$

We apply Lemma 2.7 to $w$ with $\theta \leq \frac{1}{4}$ (so that $2 \theta \leq \frac{1}{2}$ ). This gives a positive constant $C$ (independent of $\theta$ ) and a $\gamma \in(0,1)$ such that

$$
\theta^{1-m-\beta} \int_{B_{\theta}^{+}(0)} x_{m+1}^{\beta}|\nabla w|^{2} \mathrm{~d} x \leq C(2 \theta)^{2 \gamma} .
$$

Combining (4.51) and (4.52) we see that

$$
\theta^{-(1+m+\beta)} \int_{B_{\theta}^{+}(0)} x_{m+1}^{\beta}|w|^{2} \mathrm{~d} x \leq C(2 \theta)^{2 \gamma} .
$$

This holds for all fixed $\theta \in\left(0, \frac{1}{4}\right]$ and we choose $\theta<\frac{1}{2}\left(\frac{\delta}{C}\right)^{\frac{1}{2 \gamma}}$ so that (4.53) contradicts (4.50).

Remark 4.19 We could have used Lemma 2.8 in place of Lemma 2.7 to the same effect. In using the latter lemma, we observe that Hölder continuity of solutions to the linear Neumanntype problem (2.11) is sufficient to obtain energy decay and consequently Hölder continuity of minimisers of $E^{\beta}$ relative to $\mathcal{O}$; we do not need higher regularity for the linear problem at this point. 


\subsection{Energy decay}

We combine our construction of comparison maps in Sect. 4.8 with the improved control of the mean squared oscillation obtained in Sect. 4.9 in order to show that the re-scaled energy decays faster than implied by the boundary monotonicity formula, Lemma 4.5.

Lemma 4.20 Let $v \in \dot{W}_{\beta}^{1,2}\left(\mathbb{R}_{+}^{m+1} ; N\right)$ be a minimiser of $E^{\beta}$ relative to $\mathcal{O}$. Suppose $B_{R}^{+}\left(x_{0}\right)$ is a half-ball with $R \leq 1$ and $\overline{\partial^{0} B_{R}^{+}\left(x_{0}\right)} \subset \mathcal{O}$. There exist $\varepsilon_{0}=\varepsilon_{0}(m, N, \beta)>0$ and $\theta_{0}=\theta_{0}(m, N, \beta) \in\left(0, \frac{1}{4}\right)$ such that if

$$
R^{1-m-\beta} \int_{B_{R}^{+}\left(x_{0}\right)} x_{m+1}^{\beta}|\nabla v|^{2} \mathrm{~d} x \leq \varepsilon_{0},
$$

then

$$
\left(\theta_{0} r\right)^{1-m-\beta} \int_{B_{\theta_{0} r}^{+}(y)} x_{m+1}^{\beta}|\nabla v|^{2} \mathrm{~d} x \leq \frac{1}{2} r^{1-m-\beta} \int_{B_{r}^{+}(y)} x_{m+1}^{\beta}|\nabla v|^{2} \mathrm{~d} x,
$$

for every $B_{r}^{+}(y) \in \mathcal{B}^{+}\left(x_{0}, R, \frac{R}{2}\right)$.

Proof Let $B_{\rho}^{+}(y) \subset B_{r}^{+}(y) \in \mathcal{B}^{+}\left(x_{0}, R, \frac{R}{2}\right)$. Then $\rho \leq r \leq \frac{R}{2}, y \in \mathcal{O}$ and $\left|x_{0}-y\right|<\frac{R}{2}$. Suppose $v$ satisfies $R^{1-m-\beta} \int_{B_{R}^{+}\left(x_{0}\right)} x_{m+1}^{\beta}|\nabla v|^{2} \mathrm{~d} x \leq \varepsilon_{0}$ for $\varepsilon_{0}>0$ to be chosen. Then for any $\rho \in(0, r]$ the monotonicity formula, Lemma 4.5 , yields

$$
\begin{aligned}
\rho^{1-m-\beta} \int_{B_{\rho}^{+}(y)} x_{m+1}^{\beta}|\nabla v|^{2} \mathrm{~d} x & \leq r^{1-m-\beta} \int_{B_{r}^{+}(y)} x_{m+1}^{\beta}|\nabla v|^{2} \mathrm{~d} x \\
& \leq\left(\frac{R}{2}\right)^{1-m-\beta} \int_{B_{\frac{R}{2}}^{+}(y)} x_{m+1}^{\beta}|\nabla v|^{2} \mathrm{~d} x \\
& \leq C \varepsilon_{0} .
\end{aligned}
$$

We apply Lemma 4.17 on $B_{\rho}^{+}(y) \subset B_{r}^{+}(y)$, with $\rho \leq r$ to be chosen later. This gives a $\delta_{0}$ such that for any $\varepsilon \in(0,1)$, if

$$
\rho^{1-m-\beta} \int_{B_{\rho}^{+}(y)} x_{m+1}^{\beta}|\nabla v|^{2} \mathrm{~d} x \leq \delta_{0}^{2} \varepsilon^{1+m+\frac{\beta}{2}+\frac{|\beta|}{2}}
$$

then there is a $\sigma \in\left(\frac{3 \rho}{4}, \rho\right)$ such that we can find a $w_{\varepsilon} \in W_{\beta}^{1,2}\left(B_{\rho}^{+}(y) ; N\right)$ which agrees with $v$ in $B_{\rho}^{+}(y) \backslash B_{\sigma}^{+}(y)$ and satisfies

$$
\begin{aligned}
\sigma^{1-m-\beta} \int_{B_{\sigma}^{+}(y)} x_{m+1}^{\beta}|\nabla w|^{2} \mathrm{~d} x \leq & C \varepsilon \rho^{1-m-\beta} \int_{B_{\rho}^{+}(y)} x_{m+1}^{\beta}|\nabla v|^{2} \mathrm{~d} x \\
& +\frac{1}{\varepsilon} C \rho^{-(1+m+\beta)} \int_{B_{\rho}^{+}(y)} x_{m+1}^{\beta}\left|v-\bar{v}_{B_{\rho}^{+}(y), \beta}\right|^{2} \mathrm{~d} x .
\end{aligned}
$$

Assuming (4.55) and consequently (4.56) hold, we make use of the comparison property of $w$. Since $v=w$ in $B_{\rho}^{+}(y) \backslash B_{\sigma}^{+}(y)$ we may extend $w$ to an element of $\dot{W}_{\beta}^{1,2}\left(\mathbb{R}_{+}^{m+1} ; N\right)$ by requiring $w=v$ on $\mathbb{R}_{+}^{m+1} \backslash B_{\rho}^{+}(y)$. As $v$ is a minimiser of $E^{\beta}$ relative to $\mathcal{O}$, we deduce that $\int_{B_{\sigma}^{+}(y)} x_{m+1}^{\beta}|\nabla v|^{2} \mathrm{~d} x \leq \int_{B_{\sigma}^{+}(y)} x_{m+1}^{\beta}|\nabla w|^{2} \mathrm{~d} x$. Combining this fact with the monotonicity 
formula, Lemma 4.5, and (4.56) gives

$$
\begin{aligned}
\left(\frac{3 \rho}{4}\right)^{1-m-\beta} \int_{B_{\frac{3 \rho}{4}}^{+}(y)} x_{m+1}^{\beta}|\nabla v|^{2} \mathrm{~d} x \leq & C \varepsilon \rho^{1-m-\beta} \int_{B_{\rho}^{+}(y)} x_{m+1}^{\beta}|\nabla v|^{2} \mathrm{~d} x \\
& +\frac{1}{\varepsilon} C \rho^{-(1+m+\beta)} \int_{B_{\rho}^{+}(y)} x_{m+1}^{\beta}\left|v-\bar{v}_{B_{\rho}^{+}(y), \beta}\right|^{2} \mathrm{~d} x .
\end{aligned}
$$

Fix $\varepsilon=\min \left\{\frac{1}{4}, \frac{1}{4 C}\right\}$, where $C$ is the constant in (4.57) and let $\varepsilon_{0} \leq \frac{1}{C} \delta_{0}^{2} \varepsilon^{1+m+\frac{\beta}{2}+\frac{|\beta|}{2}}$ where $C$ is the constant from (4.54). It follows from (4.54) that (4.55) is satisfied and hence, substituting this $\varepsilon$ into (4.57), we have

$$
\begin{aligned}
\left(\frac{3 \rho}{4}\right)^{1-m-\beta} \int_{B_{\frac{3 \rho}{4}}^{+}(y)} x_{m+1}^{\beta}|\nabla v|^{2} \mathrm{~d} x \leq & \frac{1}{4} \rho^{1-m-\beta} \int_{B_{\rho}^{+}(y)} x_{m+1}^{\beta}|\nabla v|^{2} \mathrm{~d} x \\
& +\hat{C} \rho^{-(1+m+\beta)} \int_{B_{\rho}^{+}(y)} x_{m+1}^{\beta}\left|v-\bar{v}_{B_{\rho}^{+}(y), \beta}\right|^{2} \mathrm{~d} x
\end{aligned}
$$

for a constant $\hat{C}$ and any $\rho \leq r \leq \frac{R}{2}$. Observe that

$$
\begin{aligned}
\left|\int_{B_{r}^{+}(y)} x_{m+1}^{\beta}\langle\nabla v, \nabla \phi\rangle \mathrm{d} x\right| & =\left|\int_{B_{r}^{+}(y)} x_{m+1}^{\beta}\langle\phi, A(v)(\nabla v, \nabla v)\rangle \mathrm{d} x\right| \\
& \leq c_{0} \int_{B_{r}^{+}(y)} x_{m+1}^{\beta}|\phi||\nabla v|^{2} \mathrm{~d} x,
\end{aligned}
$$

for every $\phi \in C_{0}^{\infty}\left(B_{r}(y) ; \mathbb{R}^{n}\right)$ on every $B_{r}^{+}(y) \subset B_{R}^{+}\left(x_{0}\right)$ where $c_{0}=c_{0}(m, N)$. Hence, we may apply Lemma 4.18 for $\delta=\min \left\{\frac{1}{4}, \frac{1}{4 \hat{C}}\right\}$ and $c_{0}$ as above to obtain a corresponding $\varepsilon_{1}>0$ and $\theta_{1} \in\left(0, \frac{1}{4}\right]$ such that if $r^{1-m-\beta} \int_{B_{r}^{+}(y)} x_{m+1}^{\beta}|\nabla v|^{2} \mathrm{~d} x \leq \varepsilon_{1}$ then

$$
\begin{aligned}
& \left(\theta_{1} r\right)^{-(1+m+\beta)} \int_{B_{\theta_{1} r}^{+}(y)} x_{m+1}^{\beta}\left|v-\bar{v}_{B_{\theta_{1}}^{+}(y), \beta}\right|^{2} \mathrm{~d} x \\
& \leq \frac{1}{4 \hat{C}} r^{1-m-\beta} \int_{B_{r}^{+}(y)} x_{m+1}^{\beta}|\nabla v|^{2} \mathrm{~d} x .
\end{aligned}
$$

Now choose $\varepsilon_{0}=\frac{1}{C} \min \left\{\delta_{0}^{2} \varepsilon^{1+m+\frac{\beta}{2}+\frac{|\beta|}{2}}, \varepsilon_{1}\right\}$ where $C$ is the constant from (4.54). It follows that (4.58) and (4.59) hold on any $B_{\rho}^{+}(y) \subset B_{r}^{+}(y) \in \mathcal{B}^{+}\left(x_{0}, R, \frac{R}{2}\right)$. Thus we may apply (4.58) with $\rho=\theta_{1} r$. In turn, assuming this choice of $\rho$, we combine (4.58) with the monotonicity formula and (4.59) to see that

$$
\left(\frac{3 \theta_{1} r}{4}\right)^{1-m-\beta} \int_{\frac{B_{\frac{3 \theta_{1} r}{4}}^{+}(y)}{}} x_{m+1}^{\beta}|\nabla v|^{2} \mathrm{~d} x \leq \frac{1}{2} r^{1-m-\beta} \int_{B_{r}^{+}(y)} x_{m+1}^{\beta}|\nabla v|^{2} \mathrm{~d} x .
$$

Hence the lemma is proved with the above choice of $\varepsilon_{0}$ and $\theta_{0}=\frac{3 \theta_{1}}{4}$. 


\section{$4.11 \varepsilon$-Regularity as far as Hölder continuity}

The culmination of the results in this section so far lead to the following $\varepsilon$-regularity theorem for minimisers of $E^{\beta}$ relative to $\mathcal{O}$ which establishes the first part of Theorem 4.1.

Theorem 4.21 If $m \geq 3$, let $\beta \in(-1,1)$ and if $m=2$ let $\beta \in\left(-3^{-1}, 1\right)$. Let $v \in$ $\dot{W}_{\beta}^{1,2}\left(\mathbb{R}_{+}^{m+1} ; N\right)$ be a minimiser of $E^{\beta}$ relative to $\mathcal{O}$. Suppose $B_{R}^{+}\left(x_{0}\right)$ satisfies $R \leq 1$ and $\overline{\partial^{0} B_{R}^{+}\left(x_{0}\right)} \subset \mathcal{O}$. There exists an $\varepsilon=\varepsilon(m, N, \beta)>0$ and $a \theta=\theta(m, N, \beta) \in(0,1)$ such that if

$$
R^{1-m-\beta} \int_{B_{R}^{+}\left(x_{0}\right)} x_{m+1}^{\beta}|\nabla v|^{2} \mathrm{~d} x \leq \varepsilon
$$

then $v \in C^{0, \gamma}\left(\overline{B_{\theta R}^{+}\left(x_{0}\right)} ; N\right)$ for some $\gamma=\gamma(m, N, \beta) \in(0,1)$. In particular,

$$
\left|v\left(x_{1}\right)-v\left(x_{2}\right)\right| \leq C\left(R^{1-m-\beta} \int_{B_{R}^{+}\left(x_{0}\right)} x_{m+1}^{\beta}|\nabla v|^{2} \mathrm{~d} x\right)^{\frac{1}{2}}\left(\frac{\left|x_{1}-x_{2}\right|}{R}\right)^{\gamma}
$$

for every $x_{1}, x_{2} \in B_{\theta R}^{+}\left(x_{0}\right)$ and a constant $C=C(m, N, \beta)$.

Proof Throughout the proof we adopt the convention that all constants depend only on $m, N$ and $\beta$ unless stated otherwise. We reinforce this dependence where appropriate.

Let $v$ be a minimiser of $E^{\beta}$ relative to $\mathcal{O}$ with $R^{1-m-\beta} \int_{B_{R}^{+}\left(x_{0}\right)} x_{m+1}^{\beta}|\nabla v|^{2} \mathrm{~d} x \leq \varepsilon$ and set $\varepsilon=\min \left\{\varepsilon_{0}, \varepsilon_{1}\right\}$, where $\varepsilon_{0}$ is the number from Lemma 4.20 and $\varepsilon_{1}$ is the number from Lemma 4.9.

Observe that the function $\tilde{r} \mapsto \tilde{r}^{1-m-\beta} \int_{B_{\tilde{r}}^{+}(z)} x_{m+1}^{\beta}|\nabla v|^{2} \mathrm{~d} x$ is non-decreasing on $\left(0, \frac{R}{2}\right]$ by the monotonicity formula, Lemma 4.5. Furthermore, the choice of $\varepsilon$ allows us to apply Lemma 4.20. We apply this lemma, together with Lemma 8.23 of [15] to deduce that on every $B_{\tilde{r}}^{+}(z) \in \mathcal{B}^{+}\left(x_{0}, R, \frac{R}{2}\right)$ we have

$$
\begin{aligned}
\tilde{r}^{1-m-\beta} \int_{B_{\tilde{r}}^{+}(z)} x_{m+1}^{\beta}|\nabla v|^{2} \mathrm{~d} x & \leq C\left(2 \frac{\tilde{r}}{R}\right)^{\gamma_{0}}\left(\frac{R}{2}\right)^{1-m-\beta} \int_{B_{\frac{R}{2}}^{+}(z)} x_{m+1}^{\beta}|\nabla v|^{2} \mathrm{~d} x \\
& \leq C\left(\frac{\tilde{r}}{R}\right)^{\gamma_{0}} R^{1-m-\beta} \int_{B_{R}^{+}\left(x_{0}\right)} x_{m+1}^{\beta}|\nabla v|^{2} \mathrm{~d} x
\end{aligned}
$$

for a constant $C$ and a $\gamma_{0} \in(0,1)$ which depend only on $m, N, \beta$.

Our choice of $\varepsilon$ also permits the application of Lemma 4.9; this lemma implies that for any $B_{r}(y) \in \mathcal{B}_{\theta_{1}}\left(x_{0}, R, \frac{R}{3}\right)$, with $\theta_{1} \geq 2$ given by the lemma, and any $0<\rho \leq r$ we have

$$
\rho^{1-m} \int_{B_{\rho}(y)}|\nabla v|^{2} \mathrm{~d} x \leq C\left(\frac{\rho}{r}\right)^{\gamma_{1}} r^{1-m} \int_{B_{r}(y)}|\nabla v|^{2} \mathrm{~d} x
$$

for some $\gamma_{1} \in(0,1)$. Since $\theta_{1} \geq 2$, for any $B_{r}(y) \in \mathcal{B}_{\theta_{1}}\left(x_{0}, R, \frac{R}{3}\right)$ we have the inclusions

$$
B_{r}(y) \subset B \frac{y_{m+1}}{\theta_{1}}(y) \subset B_{\left(\frac{\theta_{1}+1}{\theta_{1}}\right) y_{m+1}}^{+}\left(y^{+}\right) \subset B_{\frac{3 y_{m+1}}{2}}^{+}\left(y^{+}\right) \in \mathcal{B}^{+}\left(x_{0}, R, \frac{R}{2}\right),
$$

where $y^{+}=y-\left(0, y_{m+1}\right)$. It follows, applying (4.6), that

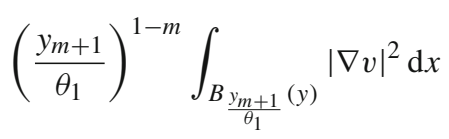




$$
\leq C\left(\frac{\left(\theta_{1}+1\right) y_{m+1}}{\theta_{1}}\right)^{1-m-\beta} \int_{B_{\left(\frac{\theta_{1}+1}{\theta_{1}}\right)_{y_{m+1}}}^{+}\left(y^{+}\right)} x_{m+1}^{\beta}|\nabla v|^{2} \mathrm{~d} x,
$$

where $C$ depends on $m, N, \beta$ and $\theta_{1}$ and thus only on $m, N, \beta$. We combine (4.62), applied with $r=\frac{y_{m+1}}{\theta_{1}}$, with (4.63), (4.64) and (4.61), applied on $B_{\tilde{r}}^{+}(z)$ with $\tilde{r}=\left(\frac{\theta_{1}+1}{\theta_{1}}\right) y_{m+1}$ and $z=y^{+}$. It follows, after defining $\hat{\gamma}=\min \left\{\gamma_{0}, \gamma_{1}\right\}$, that

$$
\rho^{1-m} \int_{B_{\rho}(y)}|\nabla v|^{2} \mathrm{~d} x \leq C\left(\frac{\rho}{R}\right)^{\hat{\gamma}} R^{1-m-\beta} \int_{B_{R}^{+}\left(x_{0}\right)} x_{m+1}^{\beta}|\nabla v|^{2} \mathrm{~d} x .
$$

This holds for any $B_{\rho}(y) \in \mathcal{B}_{\theta_{1}}\left(x_{0}, R, \frac{R}{3}\right)$. Since (4.61) holds on every $B_{\tilde{r}}^{+}(z) \in$ $\mathcal{B}^{+}\left(x_{0}, R, \frac{R}{2}\right)$ for $\gamma_{0}$, it holds on every $B_{\tilde{r}}^{+}(z) \in \mathcal{B}^{+}\left(x_{0}, R, \frac{R}{3}\right)$ for $\hat{\gamma}$. We deduce from (4.61) and (4.65) that the hypothesis of Lemma 4.8 are satisfied; applying this lemma concludes the proof.

Remark 4.22 Once we know that a minimiser of $E^{\beta}$ relative to $\mathcal{O}$ is Hölder continuous in some $B_{R}^{+}\left(x_{0}\right)$ with $\overline{\partial^{0} B_{R}^{+}\left(x_{0}\right)} \subset \mathcal{O}$, known theory for harmonic maps, see Lemma 4.13 which is from [38], implies that $v$ is smooth in $B_{R}^{+}\left(x_{0}\right)$. However, this theory does not imply $v$ is smooth up to $\partial^{0} B_{R}^{+}\left(x_{0}\right)$; henceforth our goal is essentially to prove this fact.

\subsection{An $L^{\infty}$ bound for the gradient}

The first step in our proof of higher partial regularity of locally minimising fractional harmonic maps consists of establishing an $L^{\infty}$ bound for the gradients to solutions of systems of semilinear equations with growth conditions satisfied by minimisers $v$ of $E^{\beta}$ relative to $\mathcal{O}$ and their derivatives $D^{\alpha^{\prime}} v$ where $\alpha^{\prime} \in \mathbb{N}_{0}^{m+1}$ is a multi-index with $\alpha_{m+1}^{\prime}=0$. The method of proof is that of harmonic replacement; compare the growth of the average Dirichlet energy of solutions of the semi-linear equations with that of solutions to the linearised system. We follow [38] for example. The monotonicity formulas established in Lemmas 2.8 and 2.17 are a key ingredient of the proof.

We will use the notation $|\Omega|_{\beta}=\int_{\Omega}\left|x_{m+1}\right|^{\beta} \mathrm{d} x$ and $|\Omega|=\int_{\Omega} \mathrm{d} x$ for $\Omega \subset \mathbb{R}^{m+1}$.

Lemma 4.23 Suppose $\left.v \in W_{\beta}^{1,2}\left(B_{R}^{+}\left(x_{0}\right) ; \mathbb{R}^{n}\right) \cap C^{0, \gamma} \overline{\left(B_{R}^{+}\left(x_{0}\right)\right.} ; \mathbb{R}^{n}\right)$ where $B_{R}^{+}\left(x_{0}\right)$ is a halfball with $x_{0} \in \partial \mathbb{R}_{+}^{m+1}, R \leq 1$ and $\gamma \in(0,1)$. Suppose $v$ satisfies

$$
\int_{B_{R}^{+}\left(x_{0}\right)} x_{m+1}^{\beta}\langle\nabla v, \nabla \psi\rangle \mathrm{d} x=\int_{B_{R}^{+}\left(x_{0}\right)} x_{m+1}^{\beta}\langle\psi, G(x, \nabla v)\rangle \mathrm{d} x
$$

for every $\psi \in C_{0}^{\infty}\left(B_{R}\left(x_{0}\right) ; \mathbb{R}^{n}\right)$, where $G: \mathbb{R}^{m} \times \mathbb{R}^{(m+1) n}$ is measurable and $|G(x, q)| \leq$ $C_{1}|q|^{2}+C_{2}$ for a positive $C_{1} \leq C^{*}$ and non-negative $C_{2} \leq C^{*}$ for some $C^{*}>0$. Then $\nabla v \in L^{\infty}\left(B_{\frac{R}{3}}^{+}\left(x_{0}\right) ; \mathbb{R}^{(m+1) n}\right)$ and, in particular, we have

$$
\|\nabla v\|_{L^{\infty}\left(B_{\frac{R}{3}}^{+}\left(x_{0}\right) ; \mathbb{R}^{(m+1) n}\right)}^{2} \leq C_{3} \frac{1}{\left|B_{R}^{+}\left(x_{0}\right)\right|_{\beta}} \int_{B_{R}^{+}\left(x_{0}\right)} x_{m+1}^{\beta}|\nabla v|^{2} \mathrm{~d} x+C_{4} \tilde{C}_{2}
$$

where $C_{3}=C_{3}\left(m, N, \beta, C^{*}\right), C_{4}=C_{4}\left(m, N, \beta, C^{*}\right)$ and $\tilde{C}_{2}=C_{2}^{\frac{1}{2}}+C_{2}$. In particular, if $C_{2}=0$ then $\tilde{C}_{2}=0$. 
Proof Without relabelling, we reflect $v$ evenly across the hyperplane $\partial \mathbb{R}_{+}^{m+1}$. We observe that $v \in C^{0, \gamma}\left(\overline{B_{R}\left(x_{0}\right)} ; \mathbb{R}^{n}\right) \cap W_{\beta}^{1,2}\left(B_{R}\left(x_{0}\right) ; \mathbb{R}^{n}\right)$ is a weak solution of $\operatorname{div}\left(\left|x_{m+1}\right|^{\beta} \nabla v\right)+$ $\left|x_{m+1}\right|^{\beta} \tilde{G}=0$ in $B_{R}\left(x_{0}\right)$, where $\tilde{G}$ is measurable and $|\tilde{G}(x, q)| \leq C_{1}|q|^{2}+C_{2}$. We will derive estimates on classes of $B_{\rho}(y)$ with $y \in B_{\frac{R}{2}}\left(x_{0}\right)$ and $\rho \leq \frac{R}{2}$. We focus initially on an estimate for the average energy on $B_{\frac{\rho}{2}}(y)$ in terms of that on $B_{\rho}(y)$. Since $v$ is even with respect to $\partial \mathbb{R}_{+}^{m+1}$ we only need to consider $B_{\rho}(y)$ with $y_{m+1} \geq 0$. We consider two cases, $y_{m+1}=0$ and $y_{m+1}>0$.

Suppose $B_{\rho}(y)$ is such that $B_{\rho}^{+}(y) \in \mathcal{B}^{+}\left(x_{0}, R, \frac{R}{2}\right)$. An application of Minkowski's inequality, for maps in $L_{\beta}^{2}\left(B_{\frac{\rho}{2}}(y) ; \mathbb{R}^{n(m+1)}\right)$, yields

$$
\begin{aligned}
\left(\frac{1}{\left|B_{\frac{\rho}{2}}(y)\right|_{\beta}} \int_{B_{\frac{\rho}{2}}(y)}\left|x_{m+1}\right|^{\beta}|\nabla v|^{2} \mathrm{~d} x\right)^{\frac{1}{2}} \leq & \left(\frac{1}{\left|B_{\frac{\rho}{2}}(y)\right|_{\beta}} \int_{B_{\frac{\rho}{2}}(y)}\left|x_{m+1}\right|^{\beta}|\nabla w|^{2} \mathrm{~d} x\right)^{\frac{1}{2}} \\
& +\left(\frac{C}{\left|B_{\rho}(y)\right|_{\beta}} \int_{B_{\rho}(y)}\left|x_{m+1}\right|^{\beta}|\nabla(v-w)|^{2} \mathrm{~d} x\right)^{\frac{1}{2}}
\end{aligned}
$$

for any $w \in W_{\beta}^{1,2}\left(B_{\rho}(y) ; \mathbb{R}^{n}\right)$. Let $w \in W_{\beta}^{1,2}\left(B_{\rho}(y) ; \mathbb{R}^{n}\right)$ be the weak solution of $\operatorname{div}\left(\left|x_{m+1}\right|^{\beta} \nabla w\right)=0$ in $B_{\rho}(y)$ with $w=v$ on $\partial B_{\rho}(y)$, given by Lemma 2.18. Then $w$ is smooth in $B_{\rho}(y) \backslash \partial \mathbb{R}_{+}^{m+1}$ and continuous in $\overline{B_{\rho}(y)}$. Furthermore, since $v$ is symmetric with respect to $\partial \mathbb{R}_{+}^{m+1}$, it follows from Lemma 2.19 that $w$ is symmetric with respect to $\partial \mathbb{R}_{+}^{m+1}$ and, crucially, we are now free to apply Lemma 2.8 to $w$ for every $\beta \in(-1,1)$.

As $w-v \in C\left(\overline{B_{\rho}(y)} ; \mathbb{R}^{n}\right) \cap W_{\beta, 0}^{1,2}\left(B_{\rho}(y) ; \mathbb{R}^{n}\right)$ and $v$ satisfies $\operatorname{div}\left(\left|x_{m+1}\right|^{\beta} \nabla v\right)+$ $\left|x_{m+1}\right|^{\beta} \tilde{G}=0$ and $w$ satisfies $\operatorname{div}\left(\left|x_{m+1}\right|^{\beta} \nabla w\right)=0$ weakly in $B_{\rho}(y)$, we calculate

$$
\begin{aligned}
\int_{B_{\rho}(y)}\left|x_{m+1}\right|^{\beta}|\nabla(v-w)|^{2} \mathrm{~d} x= & \int_{B_{\rho}(y)}\left|x_{m+1}\right|^{\beta}\langle v-w, \tilde{G}\rangle \mathrm{d} x \\
\leq & C_{1} \sup _{B_{\rho}(y)}|v-w| \int_{B_{\rho}(y)}\left|x_{m+1}\right|^{\beta}|\nabla v|^{2} \mathrm{~d} x \\
& +C_{2} \sup _{B_{\rho}(y)}|v-w| \int_{B_{\rho}(y)}\left|x_{m+1}\right|^{\beta} \mathrm{d} x .
\end{aligned}
$$

The Hölder continuity of $v$, together with the weak maximum and minimum principles given by Lemma 2.18 imply

$$
\sup _{B_{\rho}(y)}|v-w| \leq C \rho^{\gamma}
$$

Next we use the monotonicity and minimising properties of $w$ to scale its averaged energy. An application of Lemma 2.8, followed by an application of Lemma 2.18 yields

$$
\begin{aligned}
\frac{1}{\left|B_{\frac{\rho}{2}}(y)\right|_{\beta}} \int_{B_{\frac{\rho}{2}}(y)}\left|x_{m+1}\right|^{\beta}|\nabla w|^{2} \mathrm{~d} x & \leq \frac{1}{\left|B_{\rho}(y)\right|_{\beta}} \int_{B_{\rho}(y)}\left|x_{m+1}\right|^{\beta}|\nabla w|^{2} \mathrm{~d} x \\
& \leq \frac{1}{\left|B_{\rho}(y)\right|_{\beta}} \int_{B_{\rho}(y)}\left|x_{m+1}\right|^{\beta}|\nabla v|^{2} \mathrm{~d} x
\end{aligned}
$$


Combining (4.66)-(4.69) we see that

$$
\begin{aligned}
& \left(\frac{1}{\left|B_{\frac{\rho}{2}}(y)\right|_{\beta}} \int_{B_{\frac{\rho}{2}}(y)}\left|x_{m+1}\right|^{\beta}|\nabla v|^{2} \mathrm{~d} x\right)^{\frac{1}{2}} \leq\left(\frac{1}{\left|B_{\rho}(y)\right|_{\beta}} \int_{B_{\rho}(y)}\left|x_{m+1}\right|^{\beta}|\nabla v|^{2} \mathrm{~d} x\right)^{\frac{1}{2}} \\
& +C\left(C_{1} \rho^{\gamma} \frac{1}{\left|B_{\rho}(y)\right|_{\beta}} \int_{B_{\rho}(y)}\left|x_{m+1}\right|^{\beta}|\nabla v|^{2} \mathrm{~d} x+C_{2} \rho^{\gamma}\right)^{\frac{1}{2}} .
\end{aligned}
$$

Define $\tilde{C}_{1}=\left(C_{1}+C_{2}\right)^{\frac{1}{2}}+C_{1}$ and $\tilde{C}_{2}=C_{2}^{\frac{1}{2}}+C_{2}$. We square both sides of the preceding inequality, using Young's inequality ( $a b \leq \frac{a^{2}}{2}+\frac{b^{2}}{2}$ for $\left.a, b \geq 0\right)$ and the fact that $\gamma \in(0,1)$ and $\rho \leq R \leq 1$ to see that

$$
\begin{aligned}
& \frac{1}{\left|B_{\frac{\rho}{2}}(y)\right|_{\beta}} \int_{B_{\frac{\rho}{2}}(y)}\left|x_{m+1}\right|^{\beta}|\nabla v|^{2} \mathrm{~d} x \leq \frac{1}{\left|B_{\rho}(y)\right|_{\beta}} \int_{B_{\rho}(y)}\left|x_{m+1}\right|^{\beta}|\nabla v|^{2} \mathrm{~d} x \\
& +C\left(C_{1} \rho^{\gamma}\left(\frac{1}{\left|B_{\rho}(y)\right|_{\beta}} \int_{B_{\rho}(y)}\left|x_{m+1}\right|^{\beta}|\nabla v|^{2} \mathrm{~d} x\right)^{2}\right. \\
& \left.+C_{2} \rho^{\gamma} \frac{1}{\left|B_{\rho}(y)\right|_{\beta}} \int_{B_{\rho}(y)}\left|x_{m+1}\right|^{\beta}|\nabla v|^{2} \mathrm{~d} x\right)^{\frac{1}{2}} \\
& +C\left(C_{1} \rho^{\gamma} \frac{1}{\left|B_{\rho}(y)\right|_{\beta}} \int_{B_{\rho}(y)}\left|x_{m+1}\right|^{\beta}|\nabla v|^{2} \mathrm{~d} x+C_{2} \rho^{\gamma}\right) \\
& \leq \frac{1}{\left|B_{\rho}(y)\right|_{\beta}} \int_{B_{\rho}(y)}\left|x_{m+1}\right|^{\beta}|\nabla v|^{2} \mathrm{~d} x \\
& +C\left(\left(C_{1}+C_{2}\right) \rho^{\gamma}\left(\frac{1}{\left|B_{\rho}(y)\right|_{\beta}} \int_{B_{\rho}(y)}\left|x_{m+1}\right|^{\beta}|\nabla v|^{2} \mathrm{~d} x\right)^{2}+C_{2} \rho^{\gamma}\right)^{\frac{1}{2}} \\
& +C C_{1} \rho^{\gamma} \frac{1}{\left|B_{\rho}(y)\right|_{\beta}} \int_{B_{\rho}(y)}\left|x_{m+1}\right|^{\beta}|\nabla v|^{2} \mathrm{~d} x+C C_{2} \rho^{\gamma} \\
& \leq\left(1+C \tilde{C}_{1} \rho^{\frac{\gamma}{2}}\right) \frac{1}{\left|B_{\rho}(y)\right|_{\beta}} \int_{B_{\rho}(y)}\left|x_{m+1}\right|^{\beta}|\nabla v|^{2} \mathrm{~d} x+C \tilde{C}_{2} \rho^{\frac{\gamma}{2}} .
\end{aligned}
$$

This holds on every $B_{\rho}(y)$ with $B_{\rho}^{+}(y) \in \mathcal{B}^{+}\left(x_{0}, R, \frac{R}{2}\right)$.

Now we iterate this estimate on concentric balls. Consider $B_{\frac{R}{2}}(y)$ with $B_{\frac{R}{2}}^{+}(y) \in$ $\mathcal{B}^{+}\left(x_{0}, R, \frac{R}{2}\right)$. Let $\rho_{k}=2^{-k} \frac{R}{2}$ for $k \in \mathbb{N}_{0}$. First notice that $\rho_{k}^{\frac{\gamma}{2}} \leq 2^{-\frac{k \gamma}{2}}$. Hence

$$
\prod_{j=0}^{\infty}\left(1+C \tilde{C}_{1} \rho_{j}^{\frac{\gamma}{2}}\right) \leq \prod_{j=0}^{\infty}\left(1+C\left(\left(C^{*}\right)^{\frac{1}{2}}+C^{*}\right) 2^{-\frac{j \gamma}{2}}\right) \leq \tilde{C}<\infty
$$

where $\tilde{C}$ depends on $m, N, \beta$ and $C^{*}$. It follows from (4.70) that, for every $k \geq 1$, we have

$$
\frac{1}{\left|B_{\rho_{k}}(y)\right|_{\beta}} \int_{B_{\rho_{k}}(y)}\left|x_{m+1}\right|^{\beta}|\nabla v|^{2} \mathrm{~d} x
$$




$$
\begin{aligned}
& \leq \prod_{j=1}^{k}\left(1+C \tilde{C}_{1} \rho_{k-j}^{\frac{\gamma}{2}}\right) \frac{1}{\left|B_{\frac{R}{2}}(y)\right|_{\beta}} \int_{B_{\frac{R}{2}}(y)}\left|x_{m+1}\right|^{\beta}|\nabla v|^{2} \mathrm{~d} x \\
&+C \tilde{C}_{2} \sum_{j=1}^{k} \rho_{k-j}^{\frac{\gamma}{2}} \prod_{l=1}^{j-1}\left(1+C \tilde{C}_{1} \rho_{k-l}^{\frac{\gamma}{2}}\right) \\
& \leq C \tilde{C} \frac{1}{\left|B_{R}\left(x_{0}\right)\right|_{\beta}} \int_{B_{R}\left(x_{0}\right)}\left|x_{m+1}\right|^{\beta}|\nabla v|^{2} \mathrm{~d} x+C \tilde{C}_{2} \tilde{C} \sum_{j=1}^{k} 2^{\frac{\gamma}{2}(j-k)} \\
& \leq C \tilde{C} \frac{1}{\left|B_{R}^{+}\left(x_{0}\right)\right|_{\beta}} \int_{B_{R}^{+}\left(x_{0}\right)} x_{m+1}^{\beta}|\nabla v|^{2} \mathrm{~d} x+C \tilde{C}_{2} \tilde{C} .
\end{aligned}
$$

Now we consider estimates on $B_{\rho}(y)$ with $B \frac{y_{m+1}}{2}(y) \in \mathcal{B}\left(x_{0}, R, \frac{R}{3}\right)$; on this class of ball we have

$$
B_{\rho}(y) \subset B_{\frac{y_{m+1}}{2}}(y) \subset B_{\frac{3}{2} y_{m+1}}^{+}\left(y^{+}\right) \subset B_{\frac{R}{2}}^{+}\left(y^{+}\right) \in \mathcal{B}^{+}\left(x_{0}, R, \frac{R}{2}\right),
$$

where $y^{+}=y-\left(0, y_{m+1}\right)$. Let $w \in W_{\beta}^{1,2}\left(B_{\rho}(y) ; \mathbb{R}^{n}\right)$ be the weak solution of $\operatorname{div}\left(\left|x_{m+1}\right|^{\beta} \nabla w\right)=0$ in $B_{\rho}(y)$ with $w=v$ on $\partial B_{\rho}(y)$, given by Lemma 2.18 and suppose $\theta \geq 2$ is such that $y_{m+1} \geq \theta \rho$. Then Lemma 2.17 yields

$$
\frac{1}{\left|B_{\frac{\rho}{2}}(y)\right|} \int_{B_{\frac{\rho}{2}}(y)} x_{m+1}^{\beta}|\nabla w|^{2} \mathrm{~d} x \leq\left(1+\frac{C}{\theta-1}\right) \frac{1}{\left|B_{\rho}(y)\right|} \int_{B_{\rho}(y)} x_{m+1}^{\beta}|\nabla w|^{2} \mathrm{~d} x .
$$

Hence, repeating (4.66)-(4.69) but with $\left|B_{\frac{\rho}{2}}(y)\right|_{\beta}$ replaced by $\left|B_{\frac{\rho}{2}}(y)\right|$, we find

$$
\begin{aligned}
& \left(\frac{1}{\left|B_{\frac{\rho}{2}}(y)\right|} \int_{B_{\frac{\rho}{2}}(y)} x_{m+1}^{\beta}|\nabla v|^{2} \mathrm{~d} x\right)^{\frac{1}{2}} \leq\left(\left(1+\frac{C}{\theta-1}\right) \frac{1}{\left|B_{\rho}(y)\right|} \int_{B_{\rho}(y)} x_{m+1}^{\beta}|\nabla v|^{2} \mathrm{~d} x\right)^{\frac{1}{2}} \\
& +C\left(C_{1} \rho^{\gamma} \frac{1}{\left|B_{\rho}(y)\right|} \int_{B_{\rho}(y)} x_{m+1}^{\beta}|\nabla v|^{2} \mathrm{~d} x+C_{2} \rho^{\gamma} y_{m+1}^{\beta}\right)^{\frac{1}{2}} .
\end{aligned}
$$

We square both sides of this inequality analogously to (4.70), noting that $\theta \geq 2$ so $\frac{1}{\theta-1} \leq 1$, $\gamma \in(0,1)$ and $\rho \leq R \leq 1$, to see that

$$
\begin{aligned}
& \frac{1}{\left|B_{\frac{\rho}{2}}(y)\right|} \int_{B_{\frac{\rho}{2}}(y)} x_{m+1}^{\beta}|\nabla v|^{2} \mathrm{~d} x \\
& \quad \leq\left(1+C \tilde{C}_{1} \rho^{\frac{\gamma}{2}}+\frac{C}{\theta-1}\right) \frac{1}{\left|B_{\rho}(y)\right|} \int_{B_{\rho}(y)} x_{m+1}^{\beta}|\nabla v|^{2} \mathrm{~d} x+C \tilde{C}_{2} \rho^{\frac{\gamma}{2}} y_{m+1}^{\beta} .
\end{aligned}
$$

This holds on every $B_{\rho}(y)$ with $B \frac{y_{m+1}}{2}(y) \in \mathcal{B}\left(x_{0}, R, \frac{R}{3}\right)$ and $y_{m+1} \geq \theta \rho$.

We iterate this estimate on concentric balls. Consider $B \frac{y_{m+1}}{2}(y) \in \mathcal{B}\left(x_{0}, R, \frac{R}{3}\right)$. Let $\rho_{k}=$ $2^{-k \frac{y_{m+1}}{2}}$ for $k \in \mathbb{N}_{0}$ and observe that $y_{m+1} \geq 2^{k+1} \rho_{k}$ and $\rho_{k}^{\frac{\gamma}{2}} \leq 2^{-\frac{k \gamma}{2}}$. Observe 


$$
\begin{aligned}
\prod_{j=0}^{\infty}\left(1+C \tilde{C}_{1} \rho_{j}^{\frac{\gamma}{2}}+\frac{C}{2^{j+1}-1}\right) & \leq \prod_{j=0}^{\infty}\left(1+C\left(\left(C^{*}\right)^{\frac{1}{2}}+C^{*}\right) 2^{-\frac{j \gamma}{2}}+\frac{C}{2^{j+1}-1}\right) \\
& \leq \hat{C}<\infty
\end{aligned}
$$

where $\hat{C}$ depends on $m, N, \beta$ and $C^{*}$. It follows from (4.73) that, for every $k \geq 1$, we have

$$
\begin{aligned}
& \frac{1}{\left|B_{\rho_{k}}(y)\right|} \int_{B_{\rho_{k}}(y)} x_{m+1}^{\beta}|\nabla v|^{2} \mathrm{~d} x \\
& \leq \prod_{j=1}^{k}\left(1+C \tilde{C}_{1} \rho_{k-j}^{\frac{\gamma}{2}}+\frac{C}{2^{k-j+1}-1}\right) \frac{1}{\left|B \frac{y_{m+1}}{2}(y)\right|} \int_{B \frac{y_{m+1}}{2}(y)} x_{m+1}^{\beta}|\nabla v|^{2} \mathrm{~d} x \\
& \quad+C \tilde{C}_{2} y_{m+1}^{\beta} \sum_{j=1}^{k} \rho_{k-j}^{\frac{\gamma}{2}} \prod_{l=1}^{j-1}\left(1+C \tilde{C}_{1} \rho_{k-l}^{\frac{\gamma}{2}}+\frac{C}{2^{k-l+1}-1}\right) \\
& \leq \hat{C} \frac{1}{\left|B \frac{y_{m+1}}{2}(y)\right|} \int_{B \frac{y_{m+1}}{2}(y)} x_{m+1}^{\beta}|\nabla v|^{2} \mathrm{~d} x+C \tilde{C}_{2} \hat{C} y_{m+1}^{\beta} .
\end{aligned}
$$

Now fix $y \in B_{\frac{R}{3}}^{+}\left(x_{0}\right)$ which implies $B \frac{y_{m+1}}{2}(y) \in \mathcal{B}\left(x_{0}, R, \frac{R}{3}\right)$. We divide (4.74) by $y_{m+1}^{\beta}$, let $y^{+}=y-\left(0, y_{m+1}\right)$ and combine (4.71), (4.72) and (4.74) to see that

$$
\begin{aligned}
& \frac{y_{m+1}^{-\beta}}{\left|B_{\rho_{k}}(y)\right|} \int_{B_{\rho_{k}}(y)} x_{m+1}^{\beta}|\nabla v|^{2} \mathrm{~d} x \\
& \leq C \hat{C} \frac{y_{m+1}^{-\beta}}{\left|B \frac{y_{m+1}}{2}(y)\right|} \int_{B \frac{y_{m+1}}{2}(y)} x_{m+1}^{\beta}|\nabla v|^{2} \mathrm{~d} x+C \tilde{C}_{2} \hat{C} \\
& \leq C \hat{C} \frac{1}{\left|B_{\frac{3 y_{m+1}}{2}}\left(y^{+}\right)\right|_{\beta}} \int_{\frac{B_{3 y_{m+1}}}{2}\left(y^{+}\right)}\left|x_{m+1}\right|^{\beta}|\nabla v|^{2} \mathrm{~d} x+C \tilde{C}_{2} \hat{C} \\
& \leq C \tilde{C} \hat{C} \frac{1}{\left|B_{R}^{+}\left(x_{0}\right)\right|_{\beta}} \int_{B_{R}^{+}\left(x_{0}\right)} x_{m+1}^{\beta}|\nabla v|^{2} \mathrm{~d} x+C(\hat{C}+\hat{C} \tilde{C}) \tilde{C}_{2} .
\end{aligned}
$$

An application of Lebesgue's differentiation theorem concludes the proof.

Remark 4.24 A consequence of the preceding lemma is that Hölder continuous weak solutions of $\operatorname{div}\left(x_{m+1}^{\beta} \nabla v\right)+x_{m+1}^{\beta} G=0$ in $B_{R}^{+}\left(x_{0}\right)$ and $x_{m+1}^{\beta} \partial_{m+1} v=0$ in $\partial^{0} B_{R}^{+}\left(x_{0}\right)$, with $G$ satisfying the assumptions of the lemma on $B_{R}^{+}\left(x_{0}\right)$, are actually Lipschitz continuous on $B_{\frac{R}{3}}^{+}\left(x_{0}\right)$.

\subsection{Existence of higher order derivatives}

The existence of higher order derivatives of minimisers of $E^{\beta}$ relative to $\mathcal{O}$ in directions tangential to $\partial \mathbb{R}_{+}^{m+1}$ follows using the usual method of difference quotients.

Lemma 4.25 Fix $l \in \mathbb{N}_{0}$. Suppose $v$ is a minimiser of $E^{\beta}$ relative to $\mathcal{O}$ and let $B_{R}^{+}\left(x_{0}\right)$ be a half-ball with $\overline{\partial^{0} B_{R}^{+}\left(x_{0}\right)} \subset \mathcal{O}$. Suppose further that for every multi-index $\alpha^{\prime} \in \mathbb{N}_{0}^{m+1}$ with $\alpha_{m+1}^{\prime}=0$ and $\left|\alpha^{\prime}\right| \leq l$ we have $\left.D^{\alpha^{\prime}} v \in C^{0, \gamma} \overline{\left(B_{R}^{+}\left(x_{0}\right)\right.} ; \mathbb{R}^{n}\right) \cap W_{\beta}^{1,2}\left(B_{R}^{+}\left(x_{0}\right) ; \mathbb{R}^{n}\right)$ for some $\gamma \in(0,1)$ and $\nabla D^{\alpha^{\prime}} v \in L^{\infty}\left(B_{R}^{+}\left(x_{0}\right) ; \mathbb{R}^{(m+1) n}\right)$. Then for $\alpha^{\prime} \in \mathbb{N}_{0}^{m+1}$ with 
$\alpha_{m+1}^{\prime}=0$ and $\left|\alpha^{\prime}\right|=$ land $i=1, \ldots, m$, the weak derivative $\nabla \partial_{i} D^{\alpha^{\prime}}$ vexists and $\nabla \partial_{i} D^{\alpha^{\prime}} v \in$ $L_{\beta}^{2}\left(B_{\frac{R}{2}}^{+}\left(x_{0}\right) ; \mathbb{R}^{(m+1) n}\right)$.

Proof Without relabelling, we extend $A$ to a smooth section of $T^{*} \mathbb{R}^{n} \otimes T^{*} \mathbb{R}^{n} \otimes T \mathbb{R}^{n}$. Fix $\alpha^{\prime} \in \mathbb{N}_{0}^{m+1}$ with $\alpha_{m+1}^{\prime}=0$ and $\left|\alpha^{\prime}\right|=l$. Since $v$ is a minimiser of $E^{\beta}$ relative to $\mathcal{O}$, the regularity assumptions on $v$ and $D^{\alpha^{\prime}} v$ imply we may integrate by parts $l$ times in (4.1); for any $\phi \in C_{0}^{\infty}\left(B_{R}\left(x_{0}\right) ; \mathbb{R}^{n}\right)$ we have

$$
\int_{B_{R}^{+}\left(x_{0}\right)} x_{m+1}^{\beta}\left\langle\nabla D^{\alpha^{\prime}} v, \nabla \phi\right\rangle \mathrm{d} x=\int_{B_{R}^{+}\left(x_{0}\right)} x_{m+1}^{\beta}\left\langle\phi, D^{\alpha^{\prime}}(A(v)(\nabla v, \nabla v))\right\rangle \mathrm{d} x .
$$

Let $\eta \in C_{0}^{\infty}\left(B_{\frac{3 R}{4}}\left(x_{0}\right)\right)$ be a smooth cutoff function such that $\eta \equiv 1$ in $B_{\frac{R}{2}}\left(x_{0}\right), 1 \geq \eta \geq 0$ in $B_{\frac{3 R}{4}}\left(x_{0}\right) \backslash B_{\frac{R}{2}}\left(x_{0}\right)$ and $|\nabla \eta| \leq \frac{C}{R}$. Furthermore, let $\Delta_{i}^{h} D^{\alpha^{\prime}} v=h^{-1}\left(D^{\alpha^{\prime}} v\left(x+h e_{i}\right)-D^{\alpha^{\prime}} v(x)\right)$ be the difference quotient of $D^{\alpha^{\prime}} v$ and assume $|h|<\frac{R}{4}$. Note that, by approximation, $w=-\Delta_{i}^{-h}\left(\eta^{2} \Delta_{i}^{h} D^{\alpha^{\prime}} v\right)$ is an admissible test function for (4.75). We substitute $w$ into (4.75) and apply 'integration by parts' for difference quotients to see that

$$
\begin{aligned}
\int_{B_{R}^{+}\left(x_{0}\right)} \eta^{2} x_{m+1}^{\beta}\left|\Delta_{i}^{h} \nabla D^{\alpha^{\prime}} v\right|^{2} \mathrm{~d} x= & \int_{B_{\frac{3 R}{4}}^{+}\left(x_{0}\right)} \eta^{2} x_{m+1}^{\beta}\left\langle\Delta_{i}^{h} D^{\alpha^{\prime}} v, \Delta_{i}^{h} D^{\alpha^{\prime}}(A(v)(\nabla v, \nabla v))\right\rangle \mathrm{d} x \\
& -\int_{B_{\frac{3 R}{4}}^{+}\left(x_{0}\right)} 2 \eta x_{m+1}^{\beta}\left\langle\Delta_{i}^{h} \nabla D^{\alpha^{\prime}} v \cdot \nabla \eta, \Delta_{i}^{h} D^{\alpha^{\prime}} v\right\rangle \mathrm{d} x .
\end{aligned}
$$

We now use Young's inequality, $a b \leq \frac{a^{2}}{\delta 2}+\delta \frac{b^{2}}{2}$ for $a, b \geq 0$ and $\delta>0$, to move all of the terms involving $\Delta_{i}^{h} \nabla D^{\alpha^{\prime}} v$ on the right hand side of (4.76) to the left hand side. We calculate

$$
\begin{aligned}
-\int_{B_{\frac{3 R}{4}}^{+}\left(x_{0}\right)} 2 \eta x_{m+1}^{\beta}\left\langle\Delta_{i}^{h} \nabla D^{\alpha^{\prime}} v \cdot \nabla \eta, \Delta_{i}^{h} D^{\alpha^{\prime}} v\right\rangle \mathrm{d} x & \leq C \delta \int_{B_{R}^{+}\left(x_{0}\right)} \eta^{2} x_{m+1}^{\beta}\left|\Delta_{i}^{h} \nabla D^{\alpha^{\prime}} v\right|^{2} \mathrm{~d} x \\
& +\frac{C}{\delta} \int_{B_{R}^{+}\left(x_{0}\right)} x_{m+1}^{\beta}|\nabla \eta|^{2}\left|\Delta_{i}^{h} D^{\alpha^{\prime}} v\right|^{2} \mathrm{~d} x .
\end{aligned}
$$

We need to estimate the term involving $\Delta_{i}^{h} D^{\alpha^{\prime}}(A(v)(\nabla v, \nabla v))$ in a similar fashion. An application of the Mean Value Theorem, noting we are working on $B_{\frac{3 R}{4}}^{+}\left(x_{0}\right)$ and $|h|<\frac{R}{4}$, implies

$$
\left|\Delta_{i}^{h} D^{\alpha^{\prime}}(A(v)(\nabla v, \nabla v))\right| \leq C_{1}\left|\Delta_{i}^{h} \nabla D^{\alpha^{\prime}} v\right|+C_{2}
$$

where $C_{1}, C_{2}$ depend on $m, N, \beta$ and $\left\|\nabla D^{\tilde{\alpha}^{\prime}} v\right\|_{L^{\infty}\left(B_{R}^{+}\left(x_{0}\right) ; \mathbb{R}^{(m+1) n}\right)}$ where $\tilde{\alpha}^{\prime} \in \mathbb{N}_{0}^{m+1}$ with $\left|\tilde{\alpha}^{\prime}\right| \leq\left|\alpha^{\prime}\right|$ and $\tilde{\alpha}_{m+1}^{\prime}=0$. Hence, using Young's inequality again, we deduce

$$
\begin{aligned}
& \int_{B_{\frac{3 R}{4}}^{+}\left(x_{0}\right)} \eta^{2} x_{m+1}^{\beta}\left\langle\Delta_{i}^{h} D^{\alpha^{\prime}} v, \Delta_{i}^{h} D^{\alpha^{\prime}}(A(v)(\nabla v, \nabla v))\right\rangle \mathrm{d} x \\
& \quad \leq \delta \int_{B_{\frac{3 R}{4}}^{+}\left(x_{0}\right)} \eta^{2} x_{m+1}^{\beta}\left|\Delta_{i}^{h} \nabla D^{\alpha^{\prime}} v\right|^{2} \mathrm{~d} x+C \frac{C_{3}}{\delta} \int_{B_{\frac{3 R}{4}}^{+}\left(x_{0}\right)} x_{m+1}^{\beta} \mathrm{d} x,
\end{aligned}
$$


where $C_{3}$ depends on $m, N, \beta$ and $\left\|\nabla D^{\tilde{\alpha}^{\prime}} v\right\|_{L^{\infty}\left(B_{R}^{+}\left(x_{0}\right) ; \mathbb{R}^{(m+1) n}\right)}$ where $\tilde{\alpha}^{\prime} \in \mathbb{N}_{0}^{m+1}$ with $\left|\tilde{\alpha}^{\prime}\right| \leq$ $\left|\alpha^{\prime}\right|$ and $\tilde{\alpha}_{m+1}^{\prime}=0$. Choosing $\delta$ sufficiently small in (4.77) and (4.78) we combine these inequalities with (4.76). Since $|\nabla \eta| \leq \frac{C}{R}$ and $\eta \equiv 1$ in $B_{\frac{R}{2}}^{+}\left(x_{0}\right)$, we see that

$$
\int_{B_{\frac{R}{2}}^{+}\left(x_{0}\right)} x_{m+1}^{\beta}\left|\Delta_{i}^{h} \nabla D^{\alpha^{\prime}} v\right|^{2} \mathrm{~d} x \leq C_{4}\left(R^{-2}+1\right) \int_{B_{R}^{+}\left(x_{0}\right)} x_{m+1}^{\beta} \mathrm{d} x
$$

where $C_{4}$ depends on $m, N, \beta$ and $\left\|\nabla D^{\tilde{\alpha}^{\prime}} v\right\|_{L^{\infty}\left(B_{R}^{+}\left(x_{0}\right) ; \mathbb{R}^{(m+1) n}\right)}$ where $\tilde{\alpha}^{\prime} \in \mathbb{N}_{0}^{m+1}$ with $\left|\tilde{\alpha}^{\prime}\right| \leq$ $\left|\alpha^{\prime}\right|$ and $\tilde{\alpha}_{m+1}^{\prime}=0$. This bound is independent of $h$ with $|h|<\frac{R}{4}$. Hence by Lemma 2.11 we conclude that the weak derivative $\nabla \partial_{i} D^{\alpha^{\prime}} v$ exists and satisfies the above inequality with $\nabla \partial_{i} D^{\alpha^{\prime}} v$ in place of $\Delta_{i}^{h} \nabla D^{\alpha^{\prime}} v$. This concludes the proof.

\subsection{Caccioppoli-type inequality}

Here we show that the derivatives of minimisers of $E^{\beta}$ relative to $\mathcal{O}$ in directions tangential to $\partial \mathbb{R}_{+}^{m+1}$ all satisfy essentially the same Caccioppoli-type inequality.

Lemma 4.26 Fix $l \in \mathbb{N}_{0}$, let $v \in \dot{W}_{\beta}^{1,2}\left(\mathbb{R}_{+}^{m+1} ; N\right)$ be a minimiser of $E^{\beta}$ relative to $\mathcal{O}$ and let $B_{R}^{+}\left(x_{0}\right)$ be a half-ball with $R \leq 1$ and $\overline{\partial^{0} B_{R}^{+}\left(x_{0}\right)} \subset \mathcal{O}$. Suppose that for every multiindex $\alpha^{\prime} \in \mathbb{N}_{0}^{m+1}$ with $\alpha_{m+1}^{\prime}=0$ and $\left|\alpha^{\prime}\right| \leq l$ we have $D^{\alpha^{\prime}} v \in C^{0, \gamma}\left(\overline{B_{R}^{+}\left(x_{0}\right)} ; \mathbb{R}^{n}\right) \cap$ $W_{\beta}^{1,2}\left(B_{R}^{+}\left(x_{0}\right) ; \mathbb{R}^{n}\right)$ for some $\gamma \in(0,1)$ and $\nabla D^{\alpha^{\prime}} v \in L^{\infty}\left(B_{R}^{+}\left(x_{0}\right) ; \mathbb{R}^{(m+1) n}\right)$. Suppose further that for $\alpha^{\prime} \in \mathbb{N}_{0}^{m+1}$ with $\alpha_{m+1}^{\prime}=0$ and $\left|\alpha^{\prime}\right|=l$ and some $i \in\{1, \ldots, m\}$, we have $\nabla \partial_{i} D^{\alpha^{\prime}} v \in L_{\beta}^{2}\left(B_{R}^{+}\left(x_{0}\right) ; \mathbb{R}^{(m+1) n}\right)$. Let $B_{\rho}(y) \subset B_{R}\left(x_{0}\right)$ with $y_{m+1} \geq 0$. For each $\alpha^{\prime}$ with $\alpha_{m+1}^{\prime}=0$ and $\left|\alpha^{\prime}\right|=l$ there are constants $C=C(m, N, \beta)$ and $C_{1}, C_{2}$ which depend on $m, N, \beta$ and are comprised of polynomial functions, with no constant terms, of $\left\|\nabla D^{\tilde{\alpha}^{\prime}} v\right\|_{L^{\infty}\left(B_{\rho}(y) \cap \mathbb{R}_{+}^{m+1} ; \mathbb{R}^{(m+1) n}\right)}$, where $\tilde{\alpha}^{\prime} \in \mathbb{N}_{0}^{m+1}$ with $\left|\tilde{\alpha}^{\prime}\right| \leq\left|\alpha^{\prime}\right|=$ l and $\tilde{\alpha}_{m+1}^{\prime}=0$, such that

$$
\begin{aligned}
\int_{B_{\frac{\rho}{2}}(y) \cap \mathbb{R}_{+}^{m+1}} x_{m+1}^{\beta}\left|\nabla \partial_{i} D^{\alpha^{\prime}} v\right|^{2} \mathrm{~d} x \leq & C\left(C_{1}+\frac{1}{\rho^{2}}\right) \int_{B_{\rho}(y) \cap \mathbb{R}_{+}^{m+1}} x_{m+1}^{\beta}\left|\partial_{i} D^{\alpha^{\prime}} v-\lambda\right|^{2} \mathrm{~d} x \\
& +C_{2} \int_{B_{\rho}(y) \cap \mathbb{R}_{+}^{m+1}} x_{m+1}^{\beta} \mathrm{d} x
\end{aligned}
$$

for any $\lambda \in \mathbb{R}^{n}$.

Proof Fix $\alpha^{\prime}$ with $\alpha_{m+1}^{\prime}=0$ and $\left|\alpha^{\prime}\right|=l$. Integrating by parts $l+1$ times in (4.1) shows that for every $\psi \in C_{0}^{\infty}\left(B_{R}\left(x_{0}\right) ; \mathbb{R}^{n}\right)$, we have

$$
\int_{B_{R}^{+}\left(x_{0}\right)} x_{m+1}^{\beta}\left\langle\nabla \partial_{i} D^{\alpha^{\prime}} v, \nabla \psi\right\rangle \mathrm{d} x=\int_{B_{R}^{+}\left(x_{0}\right)} x_{m+1}^{\beta}\left\langle\psi, \partial_{i} D^{\alpha^{\prime}}(A(v)(\nabla v, \nabla v))\right\rangle \mathrm{d} x .
$$

Now, by approximation, we may choose $\psi=\eta^{2}\left(\partial_{i} D^{\alpha^{\prime}} v-\lambda\right)$ where $\lambda \in \mathbb{R}^{n}$ is a constant vector and $\eta \in C_{0}^{\infty}\left(B_{\rho}(y)\right)$ is a cutoff function with $\eta \equiv 1$ in $B_{\frac{\rho}{2}}(y), 0 \leq \eta \leq 1$, and $|\nabla \eta| \leq \frac{C}{\rho}$. We calculate 


$$
\begin{aligned}
\int_{B_{\rho}(y) \cap \mathbb{R}_{+}^{m+1}} x_{m+1}^{\beta} \eta^{2}\left|\nabla \partial_{i} D^{\alpha^{\prime}} v\right|^{2} \mathrm{~d} x \leq & \tilde{C}_{1} \int_{B_{\rho}(y) \cap \mathbb{R}_{+}^{m+1}} x_{m+1}^{\beta} \eta^{2}\left|\partial_{i} D^{\alpha^{\prime}} v-\lambda\right|\left|\nabla \partial_{i} D^{\alpha^{\prime}} v\right| \mathrm{d} x \\
& +\tilde{C}_{2} \int_{B_{\rho}(y) \cap \mathbb{R}_{+}^{m+1}} x_{m+1}^{\beta} \eta^{2}\left|\partial_{i} D^{\alpha^{\prime}} v-\lambda\right| \mathrm{d} x \\
& +C \int_{B_{\rho}(y) \cap \mathbb{R}_{+}^{m+1}} x_{m+1}^{\beta} \eta\left|\nabla \eta \| \partial_{i} D^{\alpha^{\prime}} v-\lambda\right|\left|\nabla \partial_{i} D^{\alpha^{\prime}} v\right| \mathrm{d} x,
\end{aligned}
$$

where $C=C(m, N, \beta)$ and $\tilde{C}_{1}, \tilde{C}_{2}$ depend on $m, N, \beta$ and are comprised of polynomial functions, with no constant terms, of $\left\|\nabla D^{\tilde{\alpha}^{\prime}} v\right\|_{L^{\infty}\left(B_{\rho}(y) \cap \mathbb{R}_{+}^{m+1} ; \mathbb{R}^{(m+1) n}\right)}$ where $\tilde{\alpha}^{\prime} \in \mathbb{N}_{0}^{m+1}$ with $\left|\tilde{\alpha}^{\prime}\right| \leq l$ and $\tilde{\alpha}_{m+1}^{\prime}=0$. We apply Young's inequality, $a b \leq \frac{\delta a^{2}}{2}+\frac{b^{2}}{\delta 2}$ for $a, b \geq 0$ and $\delta>0$, to each term on the right hand side of (4.80). We first apply this inequality to the term corresponding to $C$, choosing $\delta$ sufficiently small depending on $C$ and hence only on $m, N, \beta$ and recalling $|\nabla \eta| \leq \frac{C}{\rho}$ to see that

$$
\begin{aligned}
\int_{B_{\rho}(y) \cap \mathbb{R}_{+}^{m+1}} x_{m+1}^{\beta} \eta^{2}\left|\nabla \partial_{i} D^{\alpha^{\prime}} v\right|^{2} \mathrm{~d} x \leq & \tilde{C}_{1} \int_{B_{\rho}(y) \cap \mathbb{R}_{+}^{m+1}} x_{m+1}^{\beta} \eta^{2}\left|\partial_{i} D^{\alpha^{\prime}} v-\lambda\right|\left|\nabla \partial_{i} D^{\alpha^{\prime}} v\right| \mathrm{d} x \\
& +\tilde{C}_{2} \int_{B_{\rho}(y) \cap \mathbb{R}_{+}^{m+1}} x_{m+1}^{\beta} \eta^{2}\left|\partial_{i} D^{\alpha^{\prime}} v-\lambda\right| \mathrm{d} x \\
& +\frac{C}{\rho^{2}} \int_{B_{\rho}(y) \cap \mathbb{R}_{+}^{m+1}} x_{m+1}^{\beta}\left|\partial_{i} D^{\alpha^{\prime}} v-\lambda\right|^{2} \mathrm{~d} x
\end{aligned}
$$

where $\tilde{C}_{1}, \tilde{C}_{2}$ are possibly different from before but have the same structure and dependence as the constants in (4.80). Applying Young's inequality again, now to the terms in (4.81) corresponding to $\tilde{C}_{1}, \tilde{C}_{2}$ concludes the proof.

\subsection{Control of the mean squared oscillation of the derivatives on the boundary}

We prove an analogue of Lemma 4.18 for the derivatives of minimisers of $E^{\beta}$ relative to $\mathcal{O}$.

Lemma 4.27 Fix $l \in \mathbb{N}_{0}$. For every $\delta>0$ there exist numbers $\varepsilon>0, \tau \in(0,1)$ and $\theta \in\left(0, \frac{1}{4}\right]$ such that the following holds. Suppose $v \in \dot{W}_{\beta}^{1,2}\left(\mathbb{R}_{+}^{m+1} ; N\right)$ is a minimiser of $E^{\beta}$ relative to $\mathcal{O}$ with $D^{\alpha^{\prime}} v \in C^{0, \gamma}\left(\overline{B_{R}^{+}\left(x_{0}\right)} ; \mathbb{R}^{n}\right) \cap W_{\beta}^{1,2}\left(B_{R}^{+}\left(x_{0}\right) ; \mathbb{R}^{n}\right)$ for a $\gamma \in(0,1)$ and $\nabla D^{\alpha^{\prime}} v \in L^{\infty}\left(B_{R}^{+}\left(x_{0}\right) ; \mathbb{R}^{(m+1) n}\right)$ for every multi-index $\alpha^{\prime} \in \mathbb{N}_{0}^{m+1}$ with $\alpha_{m+1}^{\prime}=0$ and $\left|\alpha^{\prime}\right| \leq l$, where $B_{R}^{+}\left(x_{0}\right) \subset \mathbb{R}_{+}^{m+1}$ satisfies $R \leq 1$ and $\overline{\partial^{0} B_{R}^{+}\left(x_{0}\right)} \subset \mathcal{O}$. Suppose further that for $\alpha^{\prime} \in \mathbb{N}_{0}^{m+1}$ with $\alpha_{m+1}^{\prime}=0$ and $\left|\alpha^{\prime}\right|=l$ and some $i \in\{1, \ldots, m\}$, we have $\nabla \partial_{i} D^{\alpha^{\prime}} v \in L_{\beta}^{2}\left(B_{R}^{+}\left(x_{0}\right) ; \mathbb{R}^{(m+1) n}\right)$. If $\left|\alpha^{\prime}\right|=l$ and $\alpha_{m+1}^{\prime}=0$ and

$$
R^{1-m-\beta} \int_{B_{R}^{+}\left(x_{0}\right)} x_{m+1}^{\beta}|\nabla v|^{2} \mathrm{~d} x \leq \varepsilon^{2},
$$

then, for every $B_{r}^{+}(y) \in \mathcal{B}^{+}\left(x_{0}, R, \tau R\right)$, either

$$
r^{1-m-\beta} \int_{B_{r}^{+}(y)} x_{m+1}^{\beta}\left|\nabla \partial_{i} D^{\alpha^{\prime}} v\right|^{2} \mathrm{~d} x \leq \delta\left(\frac{r}{R^{\left|\alpha^{\prime}\right|+2}}\right)^{2}
$$

or

$$
(\theta r)^{-(1+m+\beta)} \int_{B_{\theta r}^{+}(y)} x_{m+1}^{\beta}\left|\partial_{i} D^{\alpha^{\prime}} v-{\overline{\partial_{i} D^{\alpha^{\prime}}}}_{B_{\theta r}^{+}(y), \beta}\right|^{2} \mathrm{~d} x
$$




$$
\leq \delta r^{1-m-\beta} \int_{B_{r}^{+}(y)} x_{m+1}^{\beta}\left|\nabla \partial_{i} D^{\alpha^{\prime}} v\right|^{2} \mathrm{~d} x .
$$

Proof We use a blow-up argument, analogous in spirit to the argument we used in the proof of Lemma 4.18. First we note that the statement of the lemma is invariant under rescaling and translation by any point in $\partial \mathbb{R}_{+}^{m+1}$. In particular, suppose the lemma holds for minimisers of $E^{\beta}$ relative to $\tilde{\mathcal{O}} \subset \partial \mathbb{R}_{+}^{m+1}$ whenever $\overline{\partial^{0} B_{1}^{+}(0)} \subset \tilde{\mathcal{O}}$. If the hypotheses of the lemma hold for minimisers of $E^{\beta}$ relative to $\mathcal{O}$ and $B_{R}^{+}\left(x_{0}\right)$ satisfies $R \leq 1$ and $\overline{\partial^{0} B_{R}^{+}\left(x_{0}\right)} \subset \mathcal{O}$, then applying the lemma to $v_{R}:=v\left(R \cdot+x_{0}\right)$ yields the conclusion of the lemma on $B_{R}^{+}\left(x_{0}\right)$.

We now prove the lemma when $R=1, x_{0}=0$ and $v$ is a minimiser of $E^{\beta}$ relative to $\mathcal{O}$ and $\overline{\partial^{0} B_{1}^{+}(0)} \subset \mathcal{O}$. Suppose the statement is false. Then there exists $\delta>0$ such that, for any fixed $\theta \in\left(0, \frac{1}{4}\right]$, we may find a sequence $\left(v_{k}\right)_{k \in \mathbb{N}}$ of minimisers of $E^{\beta}$ relative to $\mathcal{O}$ such that the following holds. Each $v_{k}$ satisfies $D^{\alpha^{\prime}} v_{k} \in C^{0, \gamma}\left(\overline{B_{1}^{+}(0)} ; \mathbb{R}^{n}\right) \cap W_{\beta}^{1,2}\left(B_{1}^{+}(0) ; \mathbb{R}^{n}\right)$ for a $\gamma \in(0,1)$ and $\nabla D^{\alpha^{\prime}} v_{k} \in L^{\infty}\left(B_{1}^{+}(0) ; \mathbb{R}^{(m+1) n}\right)$ for every multi-index $\alpha^{\prime} \in \mathbb{N}_{0}^{m+1}$ with $\alpha_{m+1}^{\prime}=0$ and $\left|\alpha^{\prime}\right| \leq l$. For $\alpha^{\prime} \in \mathbb{N}_{0}^{m+1}$ with $\alpha_{m+1}^{\prime}=0$ and $\left|\alpha^{\prime}\right|=l$ and a fixed $i=1, \ldots, m$, each $v_{k}$ further satisfies $\nabla \partial_{i} D^{\alpha^{\prime}} v_{k} \in L_{\beta}^{2}\left(B_{1}^{+}(0) ; \mathbb{R}^{(m+1) n}\right)$. Moreover, the $v_{k}$ satisfy

$$
\int_{B_{1}^{+}(0)} x_{m+1}^{\beta}\left|\nabla v_{k}\right|^{2} \mathrm{~d} x:=\varepsilon_{k}^{2} \rightarrow 0,
$$

and, furthermore, there exists a sequence of numbers $0<\tau_{k} \rightarrow 0^{+}$, half-balls $B_{r_{k}}^{+}\left(y_{k}\right) \in$ $\mathcal{B}^{+}\left(0,1, \tau_{k}\right)$, and numbers $0<r_{k} \leq \tau_{k} \rightarrow 0^{+}$such that

$$
r_{k}^{1-m-\beta} \int_{B_{r_{k}}^{+}\left(y_{k}\right)} x_{m+1}^{\beta}\left|\nabla \partial_{i} D^{\alpha^{\prime}} v_{k}\right|^{2} \mathrm{~d} x>\delta r_{k}^{2}
$$

and

$$
\begin{aligned}
& \left(\theta r_{k}\right)^{-(1+m+\beta)} \int_{B_{\theta r_{k}}^{+}\left(y_{k}\right)} x_{m+1}^{\beta}\left|\partial_{i} D^{\alpha^{\prime}} v_{k}-{\overline{\left(\partial_{i} D^{\alpha^{\prime}} v_{k}\right)}}_{B_{\theta r_{k}}^{+}\left(y_{k}\right), \beta}\right|^{2} \mathrm{~d} x \\
& >\delta r_{k}^{1-m-\beta} \int_{B_{r_{k}\left(y_{k}\right)}^{+}} x_{m+1}^{\beta}\left|\nabla \partial_{i} D^{\alpha^{\prime}} v_{k}\right|^{2} \mathrm{~d} x
\end{aligned}
$$

for $\alpha^{\prime}$ with $\left|\alpha^{\prime}\right|=l$ and $\alpha_{m+1}^{\prime}=0$.

Since each $v_{k}$ is a minimiser of $E^{\beta}$ relative to $\mathcal{O}$ and $v_{k} \in W_{\beta}^{1,2}\left(B_{1}^{+}(0) ; \mathbb{R}^{n}\right) \cap$ $\left.C^{0, \gamma} \overline{\left(B_{1}^{+}(0)\right.} ; \mathbb{R}^{n}\right)$ we deduce from Lemma 4.23 that each $v_{k}$ satisfies

$$
\left\|\nabla v_{k}\right\|_{L^{\infty}\left(B_{\frac{1}{3}}^{+}(0) ; \mathbb{R}^{(m+1) n}\right)}^{2} \leq C \int_{B_{1}^{+}(0)} x_{m+1}^{\beta}\left|\nabla v_{k}\right|^{2} \mathrm{~d} x \leq C \varepsilon_{k}^{2} \rightarrow 0 .
$$

The assumptions of the lemma guarantee that we may apply Lemma 4.26 on $B_{1}^{+}(0)$ with $\lambda=0$. We do so and conclude that there are constants $C=C(m, N, \beta)$ and $C_{1}, \stackrel{{ }^{6}}{C_{2}}$, which depend on $m, N, \beta$ and are comprised of a polynomial function, with no constant terms, of $\left\|\nabla v_{k}\right\|_{L^{\infty}\left(B_{\frac{1}{3}}^{+}(0) ; \mathbb{R}^{(m+1) n}\right)}$ and consequently satisfy $C_{1}, C_{2} \rightarrow 0$ as $k \rightarrow \infty$, such that for $\alpha^{\prime}$ with $\left|\alpha^{\prime}\right|=1$ and $\alpha_{m+1}^{\prime}=0$

$$
\int_{B_{\frac{1}{6}}^{+}(0)} x_{m+1}^{\beta}\left|\nabla D^{\alpha^{\prime}} v_{k}\right|^{2} \mathrm{~d} x \leq C\left(C_{1}+1\right) \int_{B_{\frac{1}{3}}^{+}(0)} x_{m+1}^{\beta}\left|D^{\alpha^{\prime}} v_{k}\right|^{2} \mathrm{~d} x+C_{2}
$$




$$
\leq C\left(C_{1}+1\right)\left\|\nabla v_{k}\right\|_{L^{\infty}\left(B_{\frac{1}{3}}^{+}(0) ; \mathbb{R}^{(m+1) n}\right)}^{2}+C_{2} \rightarrow 0
$$

as $k \rightarrow \infty$. If $l \geq 1$, integrating by parts in (4.1), we see that when $\left|\alpha^{\prime}\right|=1$ and $\alpha_{m+1}^{\prime}=0$, $D^{\alpha^{\prime}} v_{k}$ satisfies

$$
\int_{B_{\frac{1}{6}}^{+}(0)} x_{m+1}^{\beta}\left\langle\nabla D^{\alpha^{\prime}} v_{k}, \nabla \psi\right\rangle \mathrm{d} x=\int_{B_{\frac{1}{6}}^{+}(0)} x_{m+1}^{\beta}\langle\psi, G\rangle \mathrm{d} x
$$

for every $\psi \in C_{0}^{\infty}\left(B_{\frac{1}{6}}(0) ; \mathbb{R}^{n}\right)$ where, by Young's inequality, $|G(x, q)| \leq C_{3}|q|^{2}+C_{4}$ for constants $C_{3}=C_{3}(m, N, \beta)$ and $C_{4}$ which depends on $m, N, \beta$ and is comprised of a polynomial function, with no constant term, of $\left\|\nabla v_{k}\right\|_{L^{\infty}\left(B_{\frac{1}{3}}^{+}(0) ; \mathbb{R}^{(m+1) n}\right)}$ and hence $C_{4} \rightarrow 0$ as $k \rightarrow$ $\infty$. Now recall that by assumption $\left.D^{\alpha^{\prime}} v_{k} \in C^{0, \gamma} \overline{\left(B_{\frac{1}{6}}^{+}(0)\right.} ; \mathbb{R}^{(m+1) n}\right) \cap W_{\beta}^{1,2}\left(B_{\frac{1}{6}}^{+}(0) ; \mathbb{R}^{(m+1) n}\right)$ for each $\alpha^{\prime} \in \mathbb{N}^{m+1}$ with $\left|\alpha^{\prime}\right|=1$ and $\alpha_{m+1}^{\prime}=0$. Hence applying Lemma 4.23 again in conjunction with (4.86) and (4.87), we deduce that there exist positive constants $\tilde{C}_{3}=\tilde{C}_{3}(m, N, \beta)$ and $\tilde{C}_{4}$ which depends on $m, N, \beta$ and $k$ with $\tilde{C}_{4} \rightarrow 0$ as $k \rightarrow \infty$ such that

$$
\left\|\nabla D^{\alpha^{\prime}} v_{k}\right\|_{L^{\infty}\left(B_{\frac{1}{3} \frac{1}{6}}^{+}(0) ; \mathbb{R}^{(m+1) n}\right)}^{2} \leq \tilde{C}_{3} \frac{1}{\left|B_{\frac{1}{6}}^{+}(0)\right|_{\beta}} \int_{B_{\frac{1}{6}}^{+}(0)} x_{m+1}^{\beta}\left|\nabla D^{\alpha^{\prime}} v_{k}\right|^{2} \mathrm{~d} x+\tilde{C}_{4} \rightarrow 0
$$

as $k \rightarrow \infty$. Repeating the preceding process for $D^{\alpha^{\prime}} v_{k}$ with $\left|\alpha^{\prime}\right|=2$, then $\left|\alpha^{\prime}\right|=$ $3, \ldots,\left|\alpha^{\prime}\right|=l$, we see that

$$
\left\|\nabla D^{\alpha^{\prime}} v_{k}\right\|_{L^{\infty}\left(B_{\frac{1}{3} \sigma^{-\left|\alpha^{\prime}\right|}}^{+}(0) ; \mathbb{R}^{(m+1) n}\right)}^{2} \rightarrow 0
$$

as $k \rightarrow \infty$ for every $\alpha^{\prime}$ with $\left|\alpha^{\prime}\right| \leq l$ and $\alpha_{m+1}^{\prime}=0$.

Now fix $\alpha^{\prime}$ with $\left|\alpha^{\prime}\right|=l$ and $\alpha_{m+1}^{\prime}=0$. Discarding as may $v_{k}$ as necessary and re-indexing the resulting sequence we may assume that $2 \tau_{k} \leq 6^{-(l+1)}$ so that $B_{r_{k}}^{+}\left(y_{k}\right) \subset B_{2 r_{k}}^{+}\left(y_{k}\right) \subset$ $B_{2 \tau_{k}}^{+}\left(y_{k}\right) \in \mathcal{B}^{+}\left(0, \frac{1}{3} 6^{-l}, 6^{-(l+1)}\right)$ and, in particular, $B_{2 \tau_{k}}^{+}\left(y_{k}\right) \subset B_{\frac{1}{3} 6^{-l}}^{+}(0)$. Define

$$
r_{k}^{1-m-\beta} \int_{B_{r_{k}}^{+}\left(y_{k}\right)} x_{m+1}^{\beta}\left|\nabla \partial_{i} D^{\alpha^{\prime}} v_{k}\right|^{2} \mathrm{~d} x:=\tilde{\varepsilon}_{k}^{2} .
$$

Note that it is possible to show, combining Lemma 4.26 with $\lambda=0$ and (4.88), that $\tilde{\varepsilon}_{k}^{2} \rightarrow 0$, but this is not required in what follows.

We see from (4.84) and (4.85) that

$$
\tilde{\varepsilon}_{k}^{2}>\delta r_{k}^{2}
$$

and

$$
\left(\theta r_{k}\right)^{-(1+m+\beta)} \int_{B_{\theta r_{k}}^{+}\left(y_{k}\right)} x_{m+1}^{\beta}\left|\partial_{i} D^{\alpha^{\prime}} v_{k}-{\overline{\left(\partial_{i} D^{\alpha^{\prime}} v_{k}\right)}}_{B_{\theta r_{k}}^{+}\left(y_{k}\right), \beta}\right|^{2} \mathrm{~d} x>\delta \tilde{\varepsilon}_{k}^{2} .
$$

Define

$$
w_{k}(x)=\frac{\partial_{i} D^{\alpha^{\prime}} v_{k}\left(r_{k} x+y_{k}\right)-\overline{\left(\partial_{i} D^{\alpha^{\prime}} v_{k}\right)} B_{\theta r_{k}}^{+}\left(y_{k}\right), \beta}{\tilde{\varepsilon}_{k}} .
$$


Then

$$
\nabla w_{k}(x)=\frac{r_{k}}{\tilde{\varepsilon}_{k}} \nabla \partial_{i} D^{\alpha^{\prime}} v_{k}\left(r_{k} x+y_{k}\right) .
$$

Hence, using the change of variables $x \mapsto r_{k} x+y_{k}$, we find

$$
\int_{B_{1}^{+}(0)} x_{m+1}^{\beta}\left|\nabla w_{k}\right|^{2} \mathrm{~d} x=1 \text { and }{\overline{\left(w_{k}\right)}}_{B_{\theta}^{+}(0), \beta}=0 .
$$

Furthermore, after changing variables again, we deduce from (4.90) that

$$
\theta^{-(1+m+\beta)} \int_{B_{\theta}^{+}(0)} x_{m+1}^{\beta}\left|w_{k}\right|^{2} \mathrm{~d} x>\delta .
$$

The combination of (4.92) and the Poincaré inequality for $A_{2}$ weights implies $\left(w_{k}\right)_{k \in \mathbb{N}}$ is bounded in $W_{\beta}^{1,2}\left(B_{1}^{+}(0) ; \mathbb{R}^{n}\right)$. The the Rellich Compactness lemma, Lemma 2.5, therefore yields a subsequence $\left(w_{k_{j}}\right)_{j \in \mathbb{N}}$ which converges weakly in $W_{\beta}^{1,2}\left(B_{1}^{+}(0) ; \mathbb{R}^{n}\right)$ and strongly in $L_{\beta}^{2}\left(B_{1}^{+}(0) ; \mathbb{R}^{n}\right)$ to a $w \in W_{\beta}^{1,2}\left(B_{1}^{+}(0) ; \mathbb{R}^{n}\right)$.

Now we show that $w$ is a weak solution of the Neumann-type problem $(2.11)$ in $B_{1}^{+}(0)$. Let $\phi \in C_{0}^{\infty}\left(B_{1}(0) ; \mathbb{R}^{n}\right)$ and define $\tilde{\phi} \in C_{0}^{\infty}\left(B_{r_{k}}\left(y_{k}\right) ; \mathbb{R}^{n}\right)$ by $\tilde{\phi}(z)=\phi\left(\frac{z-y_{k}}{r_{k}}\right)$. We observe that $r_{k} \nabla \tilde{\phi}(z)=\nabla \phi(x)$ where $x \in B_{1}(0)$ and $z \in B_{r_{k}}\left(y_{k}\right)$ satisfy $z=r_{k} x+y_{k}$. Hence, using the change of variables $x \mapsto r_{k} x+y_{k}$ and (4.91), we find

$$
\begin{aligned}
\int_{B_{1}^{+}(0)} x_{m+1}^{\beta}\left\langle\nabla w_{k}, \nabla \phi\right\rangle \mathrm{d} x & =\frac{r_{k}}{\tilde{\varepsilon}_{k}} \int_{B_{1}^{+}(0)} x_{m+1}^{\beta}\left\langle\nabla \partial_{i} D^{\alpha^{\prime}} v_{k}\left(r_{k} x+y_{k}\right), \nabla \phi(x)\right\rangle \mathrm{d} x \\
& =\frac{r_{k}^{-m-\beta}}{\tilde{\varepsilon}_{k}} \int_{B_{r_{k}}^{+}\left(y_{k}\right)} z_{m+1}^{\beta}\left\langle\nabla \partial_{i} D^{\alpha^{\prime}} v_{k}, \nabla \phi\left(\frac{z-y_{k}}{r_{k}}\right)\right\rangle \mathrm{d} z \\
& =\frac{r_{k}^{1-m-\beta}}{\tilde{\varepsilon}_{k}} \int_{B_{r_{k}}^{+}\left(y_{k}\right)} z_{m+1}^{\beta}\left\langle\nabla \partial_{i} D^{\alpha^{\prime}} v_{k}, \nabla \tilde{\phi}\right\rangle \mathrm{d} z
\end{aligned}
$$

As $\tilde{\phi} \in C_{0}^{\infty}\left(B_{r_{k}}\left(y_{k}\right) ; \mathbb{R}^{n}\right), v_{k}$ is a minimiser of $E^{\beta}$ relative to $\mathcal{O}$ and, in view of (4.89), we have $\frac{r_{k}^{2}}{\tilde{\varepsilon}_{k}^{2}}<\frac{1}{\delta}$, it follows that

$$
\begin{aligned}
\left|\int_{B_{r_{k}}^{+}\left(y_{k}\right)} z_{m+1}^{\beta}\left\langle\nabla \partial_{i} D^{\alpha^{\prime}} v_{k}, \nabla \tilde{\phi}\right\rangle \mathrm{d} z\right| & \leq C \int_{B_{r_{k}}^{+}\left(y_{k}\right)} z_{m+1}^{\beta}\left(\left|\nabla \partial_{i} D^{\alpha^{\prime}} v_{k}\right|+1\right) \mathrm{d} z \\
& =C r_{k}^{1+m+\beta} \int_{B_{1}^{+}(0)} x_{m+1}^{\beta}\left(\left|\nabla \partial_{i} D^{\alpha^{\prime}} v_{k}\left(r_{k} x+y_{k}\right)\right|+1\right) \mathrm{d} x \\
& =C \tilde{\varepsilon}_{k} r_{k}^{m+\beta} \int_{B_{1}^{+}(0)} x_{m+1}^{\beta}\left(\left|\nabla w_{k}\right|+\frac{r_{k}}{\tilde{\varepsilon}_{k}}\right) \mathrm{d} x \\
& \leq C \tilde{\varepsilon}_{k} r_{k}^{m+\beta} \int_{B_{1}^{+}(0)} x_{m+1}^{\beta}\left(\left|\nabla w_{k}\right|+\delta^{-\frac{1}{2}}\right) \mathrm{d} x
\end{aligned}
$$

where $C$ depends on $m, N, \beta,\|\tilde{\phi}\|_{L^{\infty}\left(B_{r_{k}}^{+}\left(y_{k}\right) ; \mathbb{R}^{n}\right)}=\|\phi\|_{L^{\infty}\left(B_{1}^{+}(0) ; \mathbb{R}^{n}\right)}$ and is comprised of a polynomial, with no constant terms, of $\left\|\nabla D^{\tilde{\alpha}^{\prime}} v_{k}\right\|_{L^{\infty}\left(B_{\frac{1}{3} 6^{-l}}^{+}(0) ; \mathbb{R}^{n}\right)}$ where $\left|\tilde{\alpha}^{\prime}\right| \leq l=\left|\alpha^{\prime}\right|$ and $\tilde{\alpha}_{m+1}^{\prime}=0$ and is therefore independent of $k$ in view of (4.88). 
We combine (4.92) and (4.95) to see that, for any $\phi \in C_{0}^{\infty}\left(B_{1}(0) ; \mathbb{R}^{n}\right)$, the weak convergence of $w_{k_{j}}$ to $w$ in $W_{\beta}^{1,2}\left(B_{1}^{+}(0) ; \mathbb{R}^{n}\right)$ yields

$$
\begin{aligned}
\left|\int_{B_{1}^{+}(0)} x_{m+1}^{\beta}\langle\nabla w, \nabla \phi\rangle \mathrm{d} x\right| & =\lim _{j \rightarrow \infty}\left|\int_{B_{1}^{+}(0)} x_{m+1}^{\beta}\left\langle\nabla w_{k_{j}}, \nabla \phi\right\rangle \mathrm{d} x\right| \\
& \leq C \lim _{j \rightarrow \infty} r_{k_{j}} \int_{B_{1}^{+}(0)} x_{m+1}^{\beta}\left(\left|\nabla w_{k_{j}}\right|+\delta^{-\frac{1}{2}}\right) \mathrm{d} x \\
& =0
\end{aligned}
$$

since $r_{k_{j}} \rightarrow 0$. Hence $w$ is a weak solution of (2.11) in $B_{1}^{+}(0)$.

Using the Rellich Compactness Lemma, Lemma 2.5, we take limits in (4.92) and (4.93) to see that

$$
\int_{B_{1}^{+}(0)} x_{m+1}^{\beta}|\nabla w|^{2} \mathrm{~d} x \leq 1 \text { and } \bar{w}_{B_{\theta}^{+}(0), \beta}=0
$$

and

$$
\theta^{-(1+m+\beta)} \int_{B_{\theta}^{+}(0)} x_{m+1}^{\beta}|w|^{2} \mathrm{~d} x \geq \delta
$$

respectively. Now, in view of (4.96), the Poincaré inequality for $A_{2}$ weights yields

$$
\theta^{-(1+m+\beta)} \int_{B_{\theta}^{+}(0)} x_{m+1}^{\beta}|w|^{2} \mathrm{~d} x \leq C \theta^{1-m-\beta} \int_{B_{\theta}^{+}(0)} x_{m+1}^{\beta}|\nabla w|^{2} \mathrm{~d} x .
$$

Lastly, since $w$ is a weak solution of (2.11) we may apply Corollary 2.7 to $w$ with $\theta \leq \frac{1}{4}$ (so that $2 \theta \leq \frac{1}{2}$ ). This gives a positive constant $C$ (independent of $\theta$ ) and a $\gamma \in(0,1)$ such that

$$
\theta^{1-m-\beta} \int_{B_{\theta}^{+}(0)} x_{m+1}^{\beta}|\nabla w|^{2} \mathrm{~d} x \leq C(2 \theta)^{2 \gamma} .
$$

Combining (4.98) and (4.99) we see that

$$
\theta^{-(1+m+\beta)} \int_{B_{\theta}^{+}(0)} x_{m+1}^{\beta}|w|^{2} \mathrm{~d} x \leq C(2 \theta)^{2 \gamma} .
$$

This holds for all fixed $\theta \in\left(0, \frac{1}{4}\right]$ and we choose $\theta<2^{-1}\left(\frac{\delta}{C}\right)^{\frac{1}{2 \gamma}}$ so that (4.100) contradicts (4.97). Hence the lemma is proved.

\subsection{Control of the mean squared oscillation of the derivatives in the interior}

We need a counterpart to Lemma 4.27 which holds on a class of balls with closure contained in the interior of $\mathbb{R}_{+}^{m+1}$.

Lemma 4.28 Fix $l \in \mathbb{N}_{0}$. For every $\delta>0$ there exist numbers $\varepsilon>0, \tau \in(0,1)$ and $\theta \in\left(0, \frac{1}{4}\right]$ such that the following holds. Suppose $v \in \dot{W}_{\beta}^{1,2}\left(\mathbb{R}_{+}^{m+1} ; N\right)$ is a minimiser of $E^{\beta}$ relative to $\mathcal{O}$ with $\left.D^{\alpha^{\prime}} v \in C^{0, \gamma} \overline{\left(B_{R}^{+}\left(x_{0}\right)\right.} ; \mathbb{R}^{n}\right) \cap W_{\beta}^{1,2}\left(B_{R}^{+}\left(x_{0}\right) ; \mathbb{R}^{n}\right)$ for a $\gamma \in(0,1)$ and $\nabla D^{\alpha^{\prime}} v \in L^{\infty}\left(B_{R}^{+}\left(x_{0}\right) ; \mathbb{R}^{(m+1) n}\right)$ for every multi-index $\alpha^{\prime} \in \mathbb{N}_{0}^{m+1}$ with $\alpha_{m+1}^{\prime}=0$ and $\left|\alpha^{\prime}\right| \leq l$, where $B_{R}^{+}\left(x_{0}\right) \subset \mathbb{R}_{+}^{m+1}$ satisfies $R \leq 1$ and $\overline{\partial^{0} B_{R}^{+}\left(x_{0}\right)} \subset \mathcal{O}$. Suppose further 
that for $\alpha^{\prime} \in \mathbb{N}_{0}^{m+1}$ with $\alpha_{m+1}^{\prime}=0$ and $\left|\alpha^{\prime}\right|=l$ and some $i \in\{1, \ldots, m\}$, we have $\nabla \partial_{i} D^{\alpha^{\prime}} v \in L_{\beta}^{2}\left(B_{R}^{+}\left(x_{0}\right) ; \mathbb{R}^{(m+1) n}\right)$. If $\left|\alpha^{\prime}\right|=l$ and $\alpha_{m+1}^{\prime}=0$ and

$$
R^{1-m-\beta} \int_{B_{R}^{+}\left(x_{0}\right)} x_{m+1}^{\beta}|\nabla v|^{2} \mathrm{~d} x \leq \varepsilon^{2},
$$

then, for every $B_{r}(y) \in \mathcal{B}_{4}\left(x_{0}, R, \tau R\right)$, either

$$
r^{1-m} \int_{B_{r}(y)}\left|\nabla \partial_{i} D^{\alpha^{\prime}} v\right|^{2} \mathrm{~d} x \leq \delta\left(\frac{r}{R^{\left|\alpha^{\prime}\right|+2}}\right)^{2}
$$

or

$$
(\theta r)^{-(1+m)} \int_{B_{\theta r}(y)}\left|\partial_{i} D^{\alpha^{\prime}} v-\overline{\partial_{i} D^{\alpha^{\prime}} v_{B_{\theta r}(y)}}\right|^{2} \mathrm{~d} x \leq \delta r^{1-m} \int_{B_{r}(y)}\left|\nabla \partial_{i} D^{\alpha^{\prime}} v\right|^{2} \mathrm{~d} x .
$$

Proof The method of proof is similar to the proof of Lemma 4.27. We observe that the lemma is invariant under scaling and translation with respect to $x_{0}$ in $\partial \mathbb{R}_{+}^{m+1}$ in the same way as Lemma 4.27. Hence we assume $R=1, x_{0}=0, v$ is a minimiser of $E^{\beta}$ relative to $\mathcal{O}$ and $\overline{\partial^{0} B_{1}^{+}(0)} \subset \mathcal{O}$.

Suppose the statement is false. Then there exists $\delta>0$ such that, for any fixed $\theta \in\left(0, \frac{1}{4}\right]$, we may find a sequence $\left(v_{k}\right)_{k \in \mathbb{N}}$ of minimisers of $E^{\beta}$ relative to $\mathcal{O}$ such that the following holds. Each $v_{k}$ satisfies $\left.D^{\alpha^{\prime}} v_{k} \in C^{0, \gamma} \overline{\left(B_{1}^{+}(0)\right.} ; \mathbb{R}^{n}\right) \cap W_{\beta}^{1,2}\left(B_{1}^{+}(0) ; \mathbb{R}^{n}\right)$ for a $\gamma \in(0,1)$ and $\nabla D^{\alpha^{\prime}} v_{k} \in L^{\infty}\left(B_{1}^{+}(0) ; \mathbb{R}^{(m+1) n}\right)$ for every multi-index $\alpha^{\prime} \in \mathbb{N}_{0}^{m+1}$ with $\alpha_{m+1}^{\prime}=0$ and $\left|\alpha^{\prime}\right| \leq l$. For $\alpha^{\prime} \in \mathbb{N}_{0}^{m+1}$ with $\alpha_{m+1}^{\prime}=0$ and $\left|\alpha^{\prime}\right|=l$ and a fixed $i=1, \ldots, m$, each $v_{k}$ further satisfies $\nabla \partial_{i} D^{\alpha^{\prime}} v_{k} \in L_{\beta}^{2}\left(B_{1}^{+}(0) ; \mathbb{R}^{(m+1) n}\right)$. The $v_{k}$ also satisfy

$$
\int_{B_{1}^{+}(0)} x_{m+1}^{\beta}\left|\nabla v_{k}\right|^{2} \mathrm{~d} x:=\varepsilon_{k}^{2} \rightarrow 0 .
$$

There furthermore exists a sequence of numbers $0<\tau_{k} \rightarrow 0$, balls $B_{r_{k}}\left(y_{k}\right) \in \mathcal{B}_{4}\left(0,1, \tau_{k}\right)$, and numbers $0<r_{k} \leq \tau_{k} \rightarrow 0$ such that

$$
r_{k}^{1-m} \int_{B_{r_{k}}\left(y_{k}\right)}\left|\nabla \partial_{i} D^{\alpha^{\prime}} v_{k}\right|^{2} \mathrm{~d} x>\delta r_{k}^{2}
$$

and

$$
\begin{aligned}
& \left(\theta r_{k}\right)^{-(1+m)} \int_{B_{\theta r_{k}}\left(y_{k}\right)}\left|\partial_{i} D^{\alpha^{\prime}} v_{k}-\overline{\left(\partial_{i} D^{\alpha^{\prime}} v_{k}\right)} B_{\theta r_{k}}\left(y_{k}\right)\right|^{2} \mathrm{~d} x \\
& >\delta r_{k}^{1-m} \int_{B_{r_{k}}\left(y_{k}\right)}\left|\nabla \partial_{i} D^{\alpha^{\prime}} v_{k}\right|^{2} \mathrm{~d} x
\end{aligned}
$$

for $\alpha^{\prime}$ with $\left|\alpha^{\prime}\right|=l$ and $\alpha_{m+1}^{\prime}=0$.

Since the assumptions of the lemma are the same as the assumptions of Lemma 4.27 we still have (4.88), namely, for every $\alpha^{\prime}$ with $\left|\alpha^{\prime}\right| \leq l$ and $\alpha_{m+1}^{\prime}=0$

$$
\left\|\nabla D^{\alpha^{\prime}} v_{k}\right\|^{2}{ }_{L^{\infty}\left(B_{\frac{1}{3} \sigma^{-}\left|\alpha^{\prime}\right|}^{+}(0) ; \mathbb{R}^{(m+1) n}\right)} \rightarrow 0 .
$$

Define

$$
r_{k}^{1-m} \int_{B_{r_{k}}\left(y_{k}\right)}\left|\nabla \partial_{i} D^{\alpha^{\prime}} v_{k}\right|^{2} \mathrm{~d} x:=\tilde{\varepsilon}_{k}^{2} .
$$


Fix $\alpha^{\prime}$ with $\left|\alpha^{\prime}\right|=l$ and $\alpha_{m+1}^{\prime}=0$. Discarding as many $k$ as necessary, and re-indexing the resulting sequence to $k \in \mathbb{N}$, we may assume $2 \tau_{k} \leq 6^{-(l+1)}$ so that $B_{r_{k}}\left(y_{k}\right) \in$ $\mathcal{B}_{4}\left(0, \frac{1}{3} 6^{-l}, 6^{-(l+1)}\right)$ and, in particular, $B_{r_{k}}\left(y_{k}\right) \subset B_{\frac{1}{3} 6^{-l}}^{+}(0)$. Note that similarly to in the proof of Lemma 4.27, using Lemma 4.26 with $\lambda=0$ and (4.105) we can show $\tilde{\varepsilon}_{k}^{2} \rightarrow 0$, but this is not used in what follows. Now consider the normalised sequence

$$
w_{k}(x)=\frac{\partial_{i} D^{\alpha^{\prime}} v_{k}\left(r_{k} x+y_{k}\right)-\overline{\left(\partial_{i} D^{\alpha^{\prime}} v_{k}\right)} B_{\theta r_{k}}\left(y_{k}\right)}{\tilde{\varepsilon}_{k}} .
$$

We have

$$
\nabla w_{k}(x)=\frac{r_{k}}{\tilde{\varepsilon}_{k}} \nabla \partial_{i} D^{\alpha^{\prime}} v_{k}\left(r_{k} x+y_{k}\right) .
$$

Hence, using the change of variables $x \mapsto r_{k} x+y_{k}$, we find

$$
\int_{B_{1}(0)}\left|\nabla w_{k}\right|^{2} \mathrm{~d} x=1 \text { and }{\overline{\left(w_{k}\right)}}_{B_{\theta}(0)}=0
$$

and, also using (4.104),

$$
\theta^{-(1+m)} \int_{B_{\theta}(0)}\left|w_{k}\right|^{2} \mathrm{~d} x>\delta .
$$

As a result of (4.107) and the Poincaré inequality, we observe $\left(w_{k}\right)_{k \in \mathbb{N}}$ is bounded in $W^{1,2}\left(B_{1}(0) ; \mathbb{R}^{n}\right)$. The Rellich Compactness lemma [39, Section 1.3, Lemma 1], thus yields a subsequence $\left(w_{k_{j}}\right)_{j \in \mathbb{N}}$ which converges weakly in $W^{1,2}\left(B_{1}(0) ; \mathbb{R}^{n}\right)$ and strongly in $L^{2}\left(B_{1}(0) ; \mathbb{R}^{n}\right)$ to some $w \in W^{1,2}\left(B_{1}(0) ; \mathbb{R}^{n}\right)$.

Define $f_{k}(x)=\left(1+\left(y_{k}\right)_{m+1}^{-1} r_{k} x_{m+1}\right)^{\beta}$ for each $k \in \mathbb{N}$. Observe that $a_{k_{j}}=$ $\left(y_{k_{j}}\right)_{m+1}^{-1} r_{k_{j}} \in\left[0,4^{-1}\right]$ for every $j$, since each $B_{r_{k}}\left(y_{k}\right) \in \mathcal{B}_{4}\left(0,1, \tau_{k}\right)$. Thus there is a subsequence, which we also index with $k_{j}$, which converges to $a \in\left[0,4^{-1}\right]$. Furthermore, $\left(f_{k_{j}}\right)_{j \in \mathbb{N}}$ is uniformly bounded and equicontinuous so, by the Arzelà-Ascoli theorem, there is a uniformly convergent subsequence which we again index by $k_{j}$. Since $f_{k_{j}}(x) \rightarrow f(x)=\left(1+a x_{m+1}\right)^{\beta}$ pointwise, we must also have $f_{k_{j}} \rightarrow f$ uniformly.

Now, for $\phi \in C_{0}^{\infty}\left(B_{1}(0) ; \mathbb{R}^{n}\right)$, similar calculations to those in the proof of Lemma 4.27 yield

$$
\left|\int_{B_{1}(0)} f_{k}\left\langle\nabla w_{k}, \nabla \phi\right\rangle \mathrm{d} x\right| \leq C|| \phi \|_{L^{\infty}\left(B_{1}(0) ; \mathbb{R}^{n}\right)} r_{k} \int_{B_{1}(0)}\left|\nabla w_{k}\right|+\delta^{-\frac{1}{2}} \mathrm{~d} x \rightarrow 0
$$

as $k \rightarrow \infty$. Furthermore, as $w_{k_{j}}$ converges weakly to $w$ in $W^{1,2}\left(B_{1}(0) ; \mathbb{R}^{n}\right)$ and $f_{k_{j}} \rightarrow f$ uniformly, we conclude that

$$
\int_{B_{1}(0)} f\langle\nabla w, \nabla \phi\rangle \mathrm{d} x=\lim _{j \rightarrow \infty} \int_{B_{1}(0)} f_{k_{j}}\left\langle\nabla w_{k_{j}}, \nabla \phi\right\rangle \mathrm{d} x=0 .
$$

Hence $w$ is a weak solution of $\operatorname{div}\left(\left(1+a x_{m+1}\right)^{\beta} \nabla w\right)=0$ in $B_{1}(0)$. By linear elliptic regularity theory, $w$ is smooth in $B_{1}(0)$. We also conclude by taking limits in (4.107) and (4.108) that

$$
\int_{B_{1}(0)}|\nabla w|^{2} \mathrm{~d} x \leq 1 \text { and } \bar{w}_{B_{\theta}(0)}=0 \text { and } \theta^{-(1+m)} \int_{B_{\theta}(0)}|w|^{2} \mathrm{~d} x \geq \delta
$$


respectively using the Rellich Compactness Lemma. Since $|\nabla w|^{2}$ satisfies a mean value inequality, namely $\sup _{B_{\theta}(0)}|\nabla w|^{2} \leq C(m, \beta) \int_{B_{1}(0)}|\nabla w|^{2} \mathrm{~d} x$ as shown in Theorem 2.1 in section III of [14], we apply the Poincaré inequality and (4.111) to see that

$$
\theta^{-(1+m)} \int_{B_{\theta}(0)}|w|^{2} \mathrm{~d} x \leq C \theta^{1-m} \int_{B_{\theta}(0)}|\nabla w|^{2} \mathrm{~d} x \leq C \theta^{2} \int_{B_{1}(0)}|\nabla w|^{2} \mathrm{~d} x \leq C \theta^{2} .
$$

This holds for all fixed $\theta \in\left(0, \frac{1}{2}\right]$ and we choose $\theta<\left(\frac{\delta}{C}\right)^{\frac{1}{2}}$ so that (4.112) contradicts the last statement of (4.111).

\subsection{Higher order $\varepsilon$-regularity}

With the preceding theory in hand, we are now in a position to prove our main $\varepsilon$-regularity theorem.

Proof of Theorem 4.1 Observe that the hypothesis of the theorem are invariant with respect to the rescaling $x \mapsto R x+x_{0}$. Thus we will assume $R=1, x_{0}=0$ and $\overline{\partial^{0} B_{1}^{+}(0)} \subset \mathcal{O}$.

We use proof by strong induction. We choose $\varepsilon$ to be the number from Theorem 4.21. Then the combination of Theorem 4.21, Lemmata 4.23 and 4.25 yield a $\hat{\theta}=\hat{\theta}(m, N, \beta) \leq \frac{1}{2}$ and a $\hat{\gamma} \in(0,1)$ such that $v \in C^{0, \hat{\gamma}}\left(\overline{B_{\hat{\theta}}^{+}(0)} ; \mathbb{R}^{n}\right) \cap W_{\beta}^{1,2}\left(B_{\hat{\theta}}^{+}(0) ; \mathbb{R}^{n}\right), \nabla v \in L^{\infty}\left(B_{\hat{\theta}}^{+}(0) ; \mathbb{R}^{(m+1) n}\right)$ and $\partial_{i} v \in W_{\beta}^{1,2}\left(B_{\hat{\theta}}^{+}(0) ; \mathbb{R}^{n}\right)$ for $i=1, \ldots, m$. Now fix $l \in \mathbb{N}_{0}$. The induction hypothesis is that there exists $\tilde{\theta}=\tilde{\theta}(m, N, \beta, l) \leq \frac{1}{2}$ and a $\tilde{\gamma}=\tilde{\gamma}(m, N, \beta, l) \in(0,1)$ such that the following holds. For $\alpha^{\prime} \in \mathbb{N}_{0}^{m+1}$ with $\alpha_{m+1}^{\prime}=0$ and $\left|\alpha^{\prime}\right| \leq l$, we have $D^{\alpha^{\prime}} v \in C^{0, \tilde{\gamma}}\left(\overline{B_{\tilde{\theta}}^{+}(0)} ; \mathbb{R}^{n}\right) \cap W_{\beta}^{1,2}\left(B_{\tilde{\theta}}^{+}(0) ; \mathbb{R}^{n}\right)$ and $\nabla D^{\alpha^{\prime}} v \in L^{\infty}\left(B_{\tilde{\theta}}^{+}(0) ; \mathbb{R}^{(m+1) n}\right)$. Furthermore, when $\left|\alpha^{\prime}\right|=l$ and $\alpha_{m+1}^{\prime}=0$ we suppose $\nabla \partial_{i} D^{\alpha^{\prime}} v \in L_{\beta}^{2}\left(B_{\tilde{\theta}}^{+}(0) ; \mathbb{R}^{n(m+1)}\right)$ for $i=1, \ldots, m$. We have already observed that this is true when $l=0$. The inductive step will be to show the preceding statement holds, possibly for a different $\tilde{\theta}$ and $\tilde{\gamma}$, for $D^{\alpha^{\prime}} v$ with $\left|\alpha^{\prime}\right| \leq l+1$ and $\alpha_{m+1}^{\prime}=0$. We fix $\alpha^{\prime}$ with $\left|\alpha^{\prime}\right|=l \geq 0$ and $\alpha_{m+1}^{\prime}=0$ henceforth.

Applying Lemma 4.26, we see that

$$
\begin{aligned}
\int_{B_{\frac{\rho}{2}}(y) \cap \mathbb{R}_{+}^{m+1}} x_{m+1}^{\beta}\left|\nabla \partial_{i} D^{\alpha^{\prime}} v\right|^{2} \mathrm{~d} x \leq & C_{0}\left(C_{1}+\frac{1}{\rho^{2}}\right) \int_{B_{\rho}(y) \cap \mathbb{R}_{+}^{m+1}} x_{m+1}^{\beta}\left|\partial_{i} D^{\alpha^{\prime}} v-\lambda\right|^{2} \mathrm{~d} x \\
& +C_{2} \int_{B_{\rho}(y) \cap \mathbb{R}_{+}^{m+1}} x_{m+1}^{\beta} \mathrm{d} x
\end{aligned}
$$

for any $B_{\rho}(y) \subset B_{\tilde{\theta}}(0)$ with $y_{m+1} \geq 0$ and $i=1, \ldots, m$, where $C_{0}=C_{0}(m, N, \beta)$ and $C_{1}, C_{2}$ depend on $m, N, \beta$ and are polynomial functions, with no constant terms, of $\left\|\nabla D^{\tilde{\alpha}^{\prime}} v\right\|_{L^{\infty}\left(B_{\rho}(y) \cap \mathbb{R}_{+}^{m+1} ; \mathbb{R}^{(m+1) n}\right)}$ where $\tilde{\alpha}^{\prime} \in \mathbb{N}_{0}^{m+1}$ with $\left|\tilde{\alpha}^{\prime}\right| \leq l$ and $\tilde{\alpha}_{m+1}^{\prime}=0$. We apply Lemmata 4.27 and 4.28 , with $\delta=\frac{1}{2} \frac{1}{2^{m+2} C_{0}}$, to respectively obtain numbers $\varepsilon_{1}>0, \tau_{1} \in$ $(0,1)$ and $\theta_{1} \in\left(0, \frac{1}{4}\right]$ and $\varepsilon_{2}>0, \tau_{2} \in(0,1)$ and $\theta_{2} \in\left(0, \frac{1}{4}\right]$, depending only on $\delta$ and hence only on $m, N, \beta$, such that if $\tilde{R} \leq \tilde{\theta}$ and

$$
\tilde{R}^{1-m-\beta} \int_{B_{\tilde{R}}^{+}(0)} x_{m+1}^{\beta}|\nabla v|^{2} \mathrm{~d} x \leq \min \left\{\varepsilon_{1}^{2}, \varepsilon_{2}^{2}\right\}
$$

then either (4.82) or (4.83) holds for every $B_{r_{1}}^{+}(y) \in \mathcal{B}^{+}\left(0, \tilde{R}, \tau_{1} \tilde{R}\right)$ and either (4.101) or (4.102) holds for every $B_{r_{2}}(y) \in \mathcal{B}_{4}\left(0, \tilde{R}, \tau_{2} \tilde{R}\right)$. It follows from the proof of Theorem 4.21 , 
bearing in mind $R=1$ and $x_{0}=0$, that for every $B_{r}^{+}(y) \in \mathcal{B}^{+}\left(0,1, \frac{1}{2}\right)$ we have

$$
r^{1-m-\beta} \int_{B_{r}^{+}(y)} x_{m+1}^{\beta}|\nabla v|^{2} \mathrm{~d} x \leq C r^{\tilde{\gamma}}
$$

for some $\tilde{\gamma} \in(0,1)$ and some constant $C=C(m, N, \beta)$. In particular, this holds for $y=0$ and $r \leq \frac{1}{2}$. Hence if $\tilde{R}=\tilde{R}(m, N, \beta, l)=\left(\min \left\{\frac{\varepsilon_{1}^{2}}{C}, \frac{\varepsilon_{2}^{2}}{C},\left(\frac{\tilde{\theta}}{2}\right)^{\tilde{\gamma}}\right\}\right)^{\frac{1}{\tilde{\gamma}}}$ then (4.114) holds on $B_{\tilde{R}}^{+}(0)$. We have assumed $\tilde{R} \leq \frac{\tilde{\theta}}{2}$ so that we may later apply (4.113) with impunity on any ball or half-ball in $B_{\tilde{R}}^{+}(0)$.

First we show that (4.7) essentially holds for $\nabla \partial_{i} D^{\alpha^{\prime}} v$ on every $B_{r_{1}}^{+}(y) \in \mathcal{B}^{+}\left(0, \tilde{R}, \tau_{1} \tilde{R}\right)$. We know that (4.82) or (4.83) holds on $B_{r_{1}}^{+}(y)$. We apply (4.113) with $\lambda={\overline{\partial_{i} D^{\alpha^{\prime}} v_{B_{\theta_{1}} r_{1}}}}_{(y), \beta}$, noting that $|\lambda| \leq\left\|\nabla D^{\alpha^{\prime}} v\right\|_{L^{\infty}\left(B_{\theta_{1} r_{1}}^{+}(y) ; \mathbb{R}^{(m+1) n)}\right)}$, to see that

$$
\begin{aligned}
& \left(\frac{\theta_{1} r_{1}}{2}\right)^{1-m-\beta} \int_{B_{\frac{\theta_{1} r_{1}}{2}}^{+}(y)} x_{m+1}^{\beta}\left|\nabla \partial_{i} D^{\alpha^{\prime}} v\right|^{2} \mathrm{~d} x \\
& \leq C_{0} 2^{m+\beta-1}\left(\theta_{1} r_{1}\right)^{-(1+m+\beta)} \int_{B_{\theta_{1} r_{1}}^{+}(y)} x_{m+1}^{\beta}\left|\partial_{i} D^{\alpha^{\prime}} v-{\overline{\partial_{i} D^{\alpha^{\prime}}}}_{B_{\theta_{1} r_{1}}^{+}(y), \beta}\right|^{2} \mathrm{~d} x+C r_{1}^{2} .
\end{aligned}
$$

Hence, regardless of which of (4.82) or (4.83) holds (bearing in mind our choice of $\delta$ above), we have

$\left(\sigma_{1} r_{1}\right)^{1-m-\beta} \int_{B_{\sigma_{1} r_{1}}^{+}(y)} x_{m+1}^{\beta}\left|\nabla \partial_{i} D^{\alpha^{\prime}} v\right|^{2} \mathrm{~d} x \leq \frac{1}{2} r_{1}^{1-m-\beta} \int_{B_{r_{1}}^{+}(y)} x_{m+1}^{\beta}\left|\nabla \partial_{i} D^{\alpha^{\prime}} v\right|^{2} \mathrm{~d} x+C r_{1}^{2}$,

where $\sigma_{1}=\frac{\theta_{1}}{2}$ and $C$ depends on $\left\|\nabla D^{\tilde{\alpha}^{\prime}} v\right\|_{L^{\infty}\left(B_{\tilde{\theta}}^{+}(0) ; \mathbb{R}^{(m+1) n}\right)}$ where $\left|\tilde{\alpha}^{\prime}\right| \leq l$ with $\tilde{\alpha}_{m+1}^{\prime}=0$ and, moreover, may depend on $\tilde{R}, \theta_{1}, m, N$ and $\beta$ and hence only on $m, N, \beta, l$ as $\tilde{R}=$ $\tilde{R}(m, N, \beta, l)$ and $\theta_{1}=\theta_{1}(m, N, \beta)$. This holds for any $B_{r_{1}}^{+}(y) \in \mathcal{B}^{+}\left(0, \tilde{R}, \tau_{1} \tilde{R}\right)$. We may apply (4.116) with $r_{1}$ replaced by $\sigma_{1}^{k} r_{1}$ for every $k \in \mathbb{N}$ and iterate to see that

$$
\begin{aligned}
& \left(\sigma_{1}^{k} r_{1}\right)^{1-m-\beta} \int_{B_{\sigma_{1}^{k} r_{1}}^{+}(y)} x_{m+1}^{\beta}\left|\nabla \partial_{i} D^{\alpha^{\prime}} v\right|^{2} \mathrm{~d} x \\
& \leq \frac{1}{2^{k}} r_{1}^{1-m-\beta} \int_{B_{r_{1}}^{+}(y)} x_{m+1}^{\beta}\left|\nabla \partial_{i} D^{\alpha^{\prime}} v\right|^{2} \mathrm{~d} x+C \sum_{j=0}^{k-1} 2^{-j}\left(\sigma_{1}^{k-1-j} r_{1}\right)^{2} \\
& \leq \frac{1}{2^{k}}\left(r_{1}^{1-m-\beta} \int_{B_{r_{1}}^{+}(y)} x_{m+1}^{\beta}\left|\nabla \partial_{i} D^{\alpha^{\prime}} v\right|^{2} \mathrm{~d} x+C r_{1}^{2}\right) .
\end{aligned}
$$

Setting $\gamma_{1}=-\frac{\ln 2}{\ln \sigma_{1}} \in(0,1)$ we conclude that

$$
\begin{aligned}
& r^{1-m-\beta} \int_{B_{r}^{+}(y)} x_{m+1}^{\beta}\left|\nabla \partial_{i} D^{\alpha^{\prime}} v\right|^{2} \mathrm{~d} x \\
& \leq \sigma_{1}^{1-m-\beta-\gamma_{1}}\left(\frac{r}{r_{1}}\right)^{\gamma_{1}}\left(r_{1}{ }^{1-m-\beta} \int_{B_{r_{1}}^{+}(y)} x_{m+1}^{\beta}\left|\nabla \partial_{i} D^{\alpha^{\prime}} v\right|^{2} \mathrm{~d} x+C r_{1}^{2}\right)
\end{aligned}
$$


for any $r \leq r_{1}$, where $C$ depends on the same factors as the constant in (4.116). This holds for any $B_{r_{1}}^{+}(y) \in \mathcal{B}^{+}\left(0, \tilde{R}, \tau_{1} \tilde{R}\right)$.

We want a similar estimate for $r_{2}{ }^{1-m} \int_{B_{r_{2}}(y)}\left|\nabla \partial_{i} D^{\alpha^{\prime}} v\right|^{2} \mathrm{~d} x$ on balls $B_{r_{2}}(y) \in \mathcal{B}_{4}(0, \tilde{R}$, $\left.\tau_{2} \tilde{R}\right)$. We calculate the constants in (4.6) from Sect. 4.3 explicitly. Then our choice of $\delta$ and a similar argument which lead to (4.118) yields the existence of a $\gamma_{2}=\gamma_{2}(m, N, \beta) \in(0,1)$ such that for any $B_{r_{2}}(y) \in \mathcal{B}_{4}\left(0, \tilde{R}, \tau_{2} \tilde{R}\right)$ and any $r \leq r_{2}$ we have

$$
r^{1-m} \int_{B_{r}(y)}\left|\nabla \partial_{i} D^{\alpha^{\prime}} v\right|^{2} \mathrm{~d} x \leq \sigma_{2}^{1-m-\gamma_{2}}\left(\frac{r}{r_{2}}\right)^{\gamma_{2}}\left(r_{2}{ }^{1-m} \int_{B_{r_{2}}(y)}\left|\nabla \partial_{i} D^{\alpha^{\prime}} v\right|^{2} \mathrm{~d} x+C r_{2}^{2}\right) \text {, }
$$

where $\sigma_{2}=\frac{\theta_{2}}{2}$ and $C$ depends on the same factors as in (4.118). We now use (4.118) and (4.119) to show the hypothesis (4.7) from Lemma 4.8 is satisfied.

Define $\tau=\min \left\{\frac{\tau_{1}}{2}, \tau_{2}\right\}<\frac{1}{2}, \gamma=\min \left\{\gamma_{1}, \gamma_{2}\right\}$. We apply (4.118) with $r_{1}=\tau \tilde{R}$. It follows that for every $B_{r}^{+}(y) \in \mathcal{B}^{+}(0, \tilde{R}, \tau \tilde{R})$ we have $B_{\tau \tilde{R}}^{+}(y) \in \mathcal{B}^{+}\left(0, \tilde{R}, \tau_{1} \tilde{R}\right)$ and hence

$$
\begin{aligned}
& r^{1-m-\beta} \int_{B_{r}^{+}(y)} x_{m+1}^{\beta}\left|\nabla \partial_{i} D^{\alpha^{\prime}} v\right|^{2} \mathrm{~d} x \\
& \quad \leq C\left(\frac{r}{\tau \tilde{R}}\right)^{\gamma}\left((\tau \tilde{R})^{1-m-\beta} \int_{B_{\tau \tilde{R}}^{+}(y)} x_{m+1}^{\beta}\left|\nabla \partial_{i} D^{\alpha^{\prime}} v\right|^{2} \mathrm{~d} x+C(\tau \tilde{R})^{2}\right) .
\end{aligned}
$$

Furthermore, applying (4.113) with $\lambda=0$ and $\rho=2 \tau \tilde{R}$ implies that

$$
(\tau \tilde{R})^{1-m-\beta} \int_{B_{\tau \tilde{R}}^{+}(y)} x_{m+1}^{\beta}\left|\nabla \partial_{i} D^{\alpha^{\prime}} v\right|^{2} \mathrm{~d} x \leq C\left(1+(\tau \tilde{R})^{2}\right) \leq C,
$$

where $C$ depends on $m, N, \beta, l$ and $\left\|\nabla D^{\tilde{\alpha}^{\prime}} v\right\|_{L^{\infty}\left(B_{\tilde{\theta}}^{+}(0) ; \mathbb{R}^{(m+1) n}\right)}$ where $\left|\tilde{\alpha}^{\prime}\right| \leq l$ and $\tilde{\alpha}_{m+1}^{\prime}=0$. We combine (4.120) and (4.121) to see that for every $B_{r}^{+}(y) \in \mathcal{B}^{+}(0, \tilde{R}, \tau \tilde{R})$ we have

$$
r^{1-m-\beta} \int_{B_{r}^{+}(y)} x_{m+1}^{\beta}\left|\nabla \partial_{i} D^{\alpha^{\prime}} v\right|^{2} \mathrm{~d} x \leq C\left(\frac{r}{\tau \tilde{R}}\right)^{\gamma} \leq C_{3} r^{\gamma},
$$

where $C_{3}$ depends on $m, N, \beta, l$ and $\left\|\nabla D^{\tilde{\alpha}^{\prime}} v\right\|_{L^{\infty}\left(B_{\tilde{\theta}}^{+}(0) ; \mathbb{R}^{(m+1) n}\right)}$ where $\left|\tilde{\alpha}^{\prime}\right| \leq l$ and $\tilde{\alpha}_{m+1}^{\prime}=$ 0 .

Now let $B_{r}(y) \in \mathcal{B}_{4}\left(0, \tilde{R}, \frac{2 \tau}{3} \tilde{R}\right)$. Then $B_{r}(y) \subset B_{\frac{y_{m+1}}{4}}(y) \subset B_{\frac{3 y_{m+1}}{2}}^{+}\left(y^{+}\right) \subset B_{\tau \tilde{R}}^{+}\left(y^{+}\right) \in$ $\mathcal{B}^{+}(0, \tilde{R}, \tau \tilde{R})$, where $y^{+}=y-\left(0, y_{m+1}\right)$. Recalling again (4.6) from Sect. 4.3, we note that

$$
\begin{aligned}
& \left(\frac{y_{m+1}}{4}\right)^{1-m} \int_{\frac{B_{\frac{y_{m+1}}{4}}(y)}{4}}\left|\nabla \partial_{i} D^{\alpha^{\prime}} v\right|^{2} \mathrm{~d} x \\
& \leq C\left(\frac{3 y_{m+1}}{2}\right)^{1-m-\beta} \int_{B_{\frac{3 y_{m+1}}{2}}^{+}\left(y^{+}\right)} x_{m+1}^{\beta}\left|\nabla \partial_{i} D^{\alpha^{\prime}} v\right|^{2} \mathrm{~d} x .
\end{aligned}
$$

Since $y_{m+1} \leq \tau \tilde{R} \leq 1$, applying (4.119) on $B \frac{y_{m+1}}{4}(y) \in \mathcal{B}_{4}\left(0, \tilde{R}, \tau_{2} \tilde{R}\right)$, using (4.123), and then applying (4.122) gives

$$
r^{1-m} \int_{B_{r}(y)}\left|\nabla \partial_{i} D^{\alpha^{\prime}} v\right|^{2} \mathrm{~d} x
$$




$$
\begin{aligned}
& \leq C\left(\frac{4 r}{y_{m+1}}\right)^{\gamma}\left(\left(\frac{y_{m+1}}{4}\right)^{1-m} \int_{\left.\frac{y_{\frac{y_{m}+1}{4}}(y)}{}\left|\nabla \partial_{i} D^{\alpha^{\prime}} v\right|^{2} \mathrm{~d} x+C y_{m+1}^{2}\right)} \leq C\left(\frac{r}{y_{m+1}}\right)^{\gamma}\left(C\left(\frac{3 y_{m+1}}{2}\right)^{1-m-\beta} \int_{B_{\frac{3 y_{m+1}}{2}}^{+}\left(y^{+}\right)} x_{m+1}^{\beta}\left|\nabla \partial_{i} D^{\alpha^{\prime}} v\right|^{2} \mathrm{~d} x+C y_{m+1}^{2}\right)\right. \\
& \leq C\left(\frac{r}{y_{m+1}}\right)^{\gamma}\left(C_{3}\left(\frac{3 y_{m+1}}{2}\right)^{\gamma}+C y_{m+1}^{2}\right) \\
& \leq C_{4} r^{\gamma}
\end{aligned}
$$

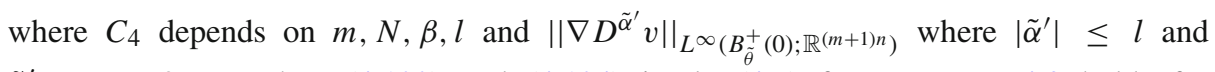
$\tilde{\alpha}_{m+1}^{\prime}=0$. Together, (4.122) and (4.124) imply (4.7) from Lemma 4.8 holds for $B_{r}^{+}(y) \in \mathcal{B}^{+}\left(0, \tilde{R}, \frac{2 \tau}{3} \tilde{R}\right)$ and $B_{r}(y) \in \mathcal{B}_{4}\left(0, \tilde{R}, \frac{2 \tau}{3} \tilde{R}\right)$. Applying this lemma shows that $\partial_{i} D^{\alpha^{\prime}} v \in C^{0, \hat{\gamma}}\left(\overline{B_{\hat{\theta}}^{+}(0)} ; \mathbb{R}^{n}\right)$ for some $\hat{\theta}, \hat{\gamma} \in(0,1)$ depending on $m, N, \beta, l$. Hence, recalling that the inductive hypothesis implies that $\partial_{i} D^{\alpha^{\prime}} v \in W_{\beta}^{1,2}\left(B_{\tilde{\theta}}^{+}(0) ; \mathbb{R}^{n}\right)$, we may apply Lemmata 4.23 and 4.25 to respectively imply $\nabla \partial_{i} D^{\alpha^{\prime}} v \in L^{\infty}\left(B_{\frac{\hat{\theta}}{3}}^{+}(0) ; \mathbb{R}^{(m+1) n}\right)$ and $\nabla \partial_{j} \partial_{i} D^{\alpha^{\prime}} v \in L_{\beta}^{2}\left(B_{\frac{\hat{\theta}}{6}}^{+}(0) ; \mathbb{R}^{n(m+1)}\right)$ for $i, j=1, \ldots, m$. This completes the inductive step and therefore the proof.

Remark 4.29 A consequence of the proof is that the number $\varepsilon$ in Theorem 4.1 can be taken to be the number from Theorem 4.21.

Theorem 4.1 yields an improvement to Theorem 4.21 for minimisers of $E^{\beta}$ relative to $\mathcal{O}$. We are now in a position to prove our partial regularity theorem.

Proof of Theorem 4.3 Aspects of the proof closely follow the proof of Theorem 3.2 in [29]. First, it follows from the theory of Schoen and Uhlenbeck [37] that there exists a set $\Sigma_{\text {int }} \subset$ $\mathbb{R}_{+}^{m+1}$, with Hausdorff dimension at most $m-2$, such that $v$ is smooth in a neighbourhood of any point in $\Sigma_{\text {int }}$.

Define

$$
\Sigma_{\text {bdry }}=\left\{y \in \mathcal{O}: \Theta_{v}^{\beta}(y) \geq \varepsilon\right\}
$$

where $\varepsilon$ is the number given by the Theorem 4.21 and $\Theta_{v}^{\beta}(y)$ is the density function defined in Remark 4.6. The upper semi-continuity of $\Theta_{v}^{\beta}$ was established in Remark 4.6 which, when combined with the definition of $\Sigma_{\text {bdry }}$, shows that $\Sigma_{\text {bdry }}$ is relatively closed in $\mathcal{O}$.

We write $\Sigma_{\text {bdry }}$ as a countable union of compact sets of the form $K \cap \Sigma_{\text {bdry }}$, where $K \subset \mathcal{O}$ is compact, and let $\Sigma^{\prime} \subset \Sigma_{\text {bdry }}$ be such a set. Fix $\delta>0$ and cover $\Sigma^{\prime}$ by a collection of balls $B_{r_{i}}^{m}\left(x_{i}\right) \subset \mathcal{O}$ with $\overline{B_{r_{i}}^{m}\left(x_{i}\right)} \subset \mathcal{O}$ with $x_{i} \in \Sigma^{\prime}$ and $0<r_{i} \leq \delta$. The compactness of $\Sigma^{\prime}$, combined with Vitali's covering theorem yields a finite subcollection of balls, $B_{r_{1}}^{m}\left(x_{1}\right), \ldots, B_{r_{I}}^{m}\left(x_{I}\right)$ for some $I \in \mathbb{N}$, of any such cover of $\Sigma^{\prime}$, which satisfies $B_{r_{i}}\left(x_{i}\right) \cap B_{r_{j}}\left(x_{j}\right)=\varnothing$ for $i \neq j, 1 \leq i, j \leq I$ and $\Sigma^{\prime} \subset \bigcup_{i=1}^{I} B_{5 r_{i}}\left(x_{i}\right)$. Using the boundary energy monotonicity formula, Lemma 4.5 , we see that

$$
\sum_{i=1}^{I}\left(10 r_{i}\right)^{m+\beta-1} \leq \frac{10^{m+\beta-1}}{\varepsilon} \sum_{i=1}^{I} \int_{B_{r_{i}}^{+}\left(x_{i}\right)} x_{m+1}^{\beta}|\nabla v|^{2} \mathrm{~d} x
$$




$$
\leq \frac{10^{m+\beta-1}}{\varepsilon} \int_{\mathcal{O} \times[0, \delta]} x_{m+1}^{\beta}|\nabla v|^{2} \mathrm{~d} x .
$$

We send $\delta \rightarrow 0^{+}$and use Lebesgue's Dominated Convergence Theorem to see that $\mathcal{H}^{m-1+\beta}\left(\Sigma^{\prime}\right)=0$ and hence $\mathcal{H}^{m-1+\beta}\left(\Sigma_{\text {bdry }}\right)=0$.

Let $x_{0} \in\left(\mathbb{R}_{+}^{m+1} \cup \mathcal{O}\right) \backslash \Sigma$. If $x_{0} \in \mathbb{R}_{+}^{m+1}$ then $x_{0} \in \mathbb{R}_{+}^{m+1} \backslash \Sigma_{\text {int }}$ and $v$ is smooth in an open ball centred at $x_{0}$ and contained in $\mathbb{R}_{+}^{m+1} \backslash \Sigma_{\text {int }} \subset\left(\mathbb{R}_{+}^{m+1} \cup \mathcal{O}\right) \backslash \Sigma$. If $x_{0} \in \mathcal{O}$ then $x_{0} \in \mathcal{O} \backslash \Sigma_{\text {bdry }}$ and $\Theta_{v}^{\beta}\left(x_{0}\right)<\varepsilon$ which, combined with the fact that $\mathcal{O} \backslash \Sigma_{\text {bdry }}$ is open in $\mathcal{O}$, implies there exists an $R>0$ such that $R^{1-m-\beta} \int_{B_{R}^{+}\left(x_{0}\right)} x_{m+1}^{\beta}|\nabla v|^{2} \mathrm{~d} x \leq \varepsilon, R \leq$ 1 and $\overline{\partial^{0} B_{R}^{+}\left(x_{0}\right)} \subset \mathcal{O} \backslash \Sigma_{\text {bdry }}$. Consequently, Theorem 4.21 implies that there are $\theta, \gamma \in$ $(0,1)$ such that $\left.v \in C^{0, \gamma} \overline{\left(B_{\theta R}^{+}\left(x_{0}\right)\right.} ; N\right)$. Furthermore, we deduce from (4.61) in the proof of Theorem 4.21 that

$$
r^{1-m-\beta} \int_{B_{r}^{+}(z)} x_{m+1}^{\beta}|\nabla v|^{2} \mathrm{~d} x \leq C\left(\frac{r}{R}\right)^{\gamma} \varepsilon
$$

on every $B_{r}^{+}(z) \in \mathcal{B}^{+}\left(x_{0}, R, \frac{R}{2}\right)$ which shows that $\Theta_{v}^{\beta}(z)=0$ for every $z \in \partial^{0} B_{\frac{R}{2}}^{+}\left(x_{0}\right)$. Now setting $\sigma=\min \left\{\theta, \frac{1}{2}\right\}$ we see that $\Theta_{v}^{\beta}(z)=0$ for $z \in \partial^{0} B_{\sigma R}^{+}\left(x_{0}\right)$ which implies $\partial^{0} B_{\sigma R}^{+}\left(x_{0}\right) \subset \mathcal{O} \backslash \Sigma_{\text {bdry }}$. Furthermore, $v$ is a Hölder continuous weakly harmonic map in any $B_{r}(y)$ with $\overline{B_{r}(y)} \subset B_{\sigma R}^{+}\left(x_{0}\right)$. We apply Lemma 4.13 to see that $v$ is smooth in $B_{\sigma R}^{+}\left(x_{0}\right)$ and conclude that $B_{\sigma R}^{+}\left(x_{0}\right) \subset \mathbb{R}_{+}^{m+1} \backslash \Sigma_{\text {int }}$. Consequently, we have $B_{\sigma R}^{+}\left(x_{0}\right) \cup \partial^{0} B_{\sigma R}^{+}\left(x_{0}\right) \subset$ $\left(\mathbb{R}_{+}^{m+1} \cup \mathcal{O}\right) \backslash \Sigma$. Note that $B_{\sigma R}^{+}\left(x_{0}\right) \cup \partial^{0} B_{\sigma R}^{+}\left(x_{0}\right)$ is an open ball centred at $x_{0}$ in the (Euclidean) topology of $\mathbb{R}_{+}^{m+1} \cup \mathcal{O}$. Hence $\Sigma$ is relatively closed in $\mathbb{R}_{+}^{m+1} \cup \mathcal{O}$. As the Hausdorff dimension of $\Sigma_{\text {int }}$ is at most $m-2<m-1+\beta$ and $\mathcal{H}^{m-1+\beta}\left(\Sigma_{\text {bdry }}\right)=0$, we deduce that $\mathcal{H}^{m+\beta-1}(\Sigma)=$ 0 . We also conclude $v \in C_{\text {loc }}^{0, \gamma}\left(\left(\mathbb{R}_{+}^{m+1} \cup \mathcal{O}\right) \backslash \Sigma ; N\right)$.

Consider $x_{0} \in\left(\mathbb{R}_{+}^{m+1} \cup \mathcal{O}\right) \backslash \Sigma$ with $x_{0} \in \mathcal{O}$. Then, as above, we observe there is an $R>0$ such that $R^{1-m-\beta} \int_{B_{R}^{+}\left(x_{0}\right)} x_{m+1}^{\beta}|\nabla v|^{2} \mathrm{~d} x \leq \varepsilon, R \leq 1$ and $\overline{\partial^{0} B_{R}^{+}\left(x_{0}\right)} \subset \mathcal{O} \backslash \Sigma_{\text {bdry }}$. In view of Remark 4.29, Theorem 4.1 implies that for every $l \in \mathbb{N}_{0}$ there exist $\theta, \gamma \in(0,1)$ such that for every $\alpha^{\prime} \in \mathbb{N}_{0}^{m+1}$ with $\left|\alpha^{\prime}\right| \leq l$ and $\alpha_{m+1}^{\prime}=0$ we have $\left.D^{\alpha^{\prime}} v \in C^{0, \gamma} \overline{\left(B_{\theta R}^{+}\left(x_{0}\right)\right.} ; \mathbb{R}^{n}\right)$. However, we also know that $\left(\mathbb{R}_{+}^{m+1} \cup \mathcal{O}\right) \backslash \Sigma$ is open in $\mathbb{R}_{+}^{m+1} \cup \mathcal{O}$. Hence there exists $\tilde{R}>0$ such that $B_{\tilde{R}}^{+}\left(x_{0}\right) \cup \partial^{0} B_{\tilde{R}}^{+}\left(x_{0}\right) \subset\left(\mathbb{R}_{+}^{m+1} \cup \mathcal{O}\right) \backslash \Sigma$. Setting $r=\min \{\theta R, \tilde{R}\}$ we conclude $D^{\alpha^{\prime}} v \in C^{0, \gamma}\left(\overline{B_{r}^{+}\left(x_{0}\right)} ; \mathbb{R}^{n}\right)$ and $B_{r}^{+}\left(x_{0}\right) \cup \partial^{0} B_{r}^{+}\left(x_{0}\right) \subset\left(\mathbb{R}_{+}^{m+1} \cup \mathcal{O}\right) \backslash \Sigma$. We iteratively apply Lemmata 4.23 and 4.25 to see that for every $\alpha^{\prime} \in \mathbb{N}_{0}^{m+1}$ with $\left|\alpha^{\prime}\right| \leq l$ and $\alpha_{m+1}^{\prime}=0$ we have $\nabla D^{\alpha^{\prime}} v \in L^{\infty}\left(B_{\tilde{r}}^{+}\left(x_{0}\right) ; \mathbb{R}^{(m+1) n}\right)$ and $D^{\alpha^{\prime}} v \in W_{\beta}^{1,2}\left(B_{\tilde{r}}^{+}\left(x_{0}\right) ; \mathbb{R}^{n}\right)$ for some $\tilde{r} \leq r$. It follows that $B_{\tilde{r}}^{+}\left(x_{0}\right) \cup \partial^{0} B_{\tilde{r}}^{+}\left(x_{0}\right) \subset\left(\mathbb{R}_{+}^{m+1} \cup \mathcal{O}\right) \backslash \Sigma$. Hence $v \in C_{\text {loc }}^{0,1}\left(\left(\mathbb{R}_{+}^{m+1} \cup \mathcal{O}\right) \backslash \Sigma ; N\right)$ and for every multi-index $\alpha^{\prime} \in \mathbb{N}^{m+1}$ with $\alpha_{m+1}^{\prime}=0$ we have $D^{\alpha^{\prime}} v \in C_{\text {loc }}^{0,1}\left(\left(\mathbb{R}_{+}^{m+1} \cup \mathcal{O}\right) \backslash \Sigma ; \mathbb{R}^{n}\right)$ and $\nabla D^{\alpha^{\prime}} v \in L_{l o c}^{\infty}\left(\left(\mathbb{R}_{+}^{m+1} \cup \mathcal{O}\right) \backslash \Sigma ; \mathbb{R}^{(m+1) n}\right)$.

Lastly, for $x_{0}$ as in the preceding paragraph, fix $\alpha^{\prime} \in \mathbb{N}_{0}^{m+1}$ with $\alpha_{m+1}^{\prime}=0$ and $\tilde{r} \leq 1$ such that $B_{\tilde{r}}^{+}\left(x_{0}\right) \cup \partial^{0} B_{\tilde{r}}^{+}\left(x_{0}\right) \subset\left(\mathbb{R}_{+}^{m+1} \cup \mathcal{O}\right) \backslash \Sigma$. Making $\tilde{r}$ smaller if necessary, we may assume that $\nabla D^{\tilde{\alpha}^{\prime}} v \in L^{\infty}\left(B_{\tilde{r}}^{+}\left(x_{0}\right) ; \mathbb{R}^{(m+1) n}\right)$ and ${D^{\alpha^{\prime}}} v \in C^{0,1} \overline{\left(B_{\tilde{r}}^{+}\left(x_{0}\right)\right.} ; \mathbb{R}^{n})$ for every $\tilde{\alpha}^{\prime} \in \mathbb{N}_{0}^{m+1}$ with $\tilde{\alpha}_{m+1}^{\prime}=0$ and $\left|\tilde{\alpha}^{\prime}\right| \leq\left|\alpha^{\prime}\right|+2$. We also observe that since $\left.v \in C^{0,1} \overline{\left(B_{\tilde{r}}^{+}\left(x_{0}\right)\right.} ; N\right)$ is a Hölder continuous harmonic map, it is smooth in $B_{\tilde{r}}^{+}\left(x_{0}\right)$ by Lemma 4.13 and so we have $x_{m+1}^{-\beta} \partial_{m+1}\left(x_{m+1}^{\beta} \partial_{m+1} D^{\alpha^{\prime}} v\right)=-\left(\Delta^{\prime} D^{\alpha^{\prime}} v+D^{\alpha^{\prime}}(A(v)(\nabla v, \nabla v))\right)$ classi- 
cally in $B_{\tilde{r}}^{+}\left(x_{0}\right)$, where $\Delta^{\prime}$ is the Laplacian with respect to $x_{i}, i=1, \ldots, m$. Hence, $x_{m+1}^{-\beta} \partial_{m+1}\left(x_{m+1}^{\beta} \partial_{m+1} D^{\alpha^{\prime}} v\right)$ is bounded in $B_{\tilde{r}}^{+}\left(x_{0}\right)$. Hence, for every $B_{\rho}^{+}(y) \in \mathcal{B}^{+}\left(x_{0}, \frac{\tilde{r}}{3}, \frac{\tilde{r}}{6}\right)$ we calculate

$$
\rho^{1-m+\beta} \int_{B_{\rho}^{+}(y)} x_{m+1}^{-\beta}\left|\nabla\left(x_{m+1}^{\beta} \partial_{m+1} D^{\alpha^{\prime}} v\right)\right|^{2} \mathrm{~d} x \leq C \rho^{2+2 \beta} .
$$

Moreover, for every $B_{\rho}(y) \in \mathcal{B}\left(x_{0}, \frac{\tilde{r}}{3}, \frac{\tilde{r}}{6}\right)$ we calculate

$$
\rho^{1-m} \int_{B_{\rho}(y)}\left|\nabla\left(x_{m+1}^{\beta} \partial_{m+1} D^{\alpha^{\prime}} v\right)\right|^{2} \mathrm{~d} x \leq C \rho^{2-2|\beta|} .
$$

An application of Lemma 4.8 concludes the proof.

Acknowledgements This work is based on the authors $\mathrm{PhD}$ thesis, although improvements of the results regarding higher regularity have been made for this article. Much of this work was conducted whilst the author was a postgraduate student at the University of Bath under the supervision of Professor Roger Moser. The author would like to thank Professor Moser for the opportunity to work on this project, for many interesting and helpful discussions about this work and for his unwavering support. The author is also grateful to the referees for their feedback.

Open Access This article is distributed under the terms of the Creative Commons Attribution 4.0 International License (http://creativecommons.org/licenses/by/4.0/), which permits unrestricted use, distribution, and reproduction in any medium, provided you give appropriate credit to the original author(s) and the source, provide a link to the Creative Commons license, and indicate if changes were made.

\section{References}

1. Bethuel, F.: A new obstruction to the extension problem for Sobolev maps between manifolds. J. Fixed Point Theory Appl. 15(1), 155-183 (2014). https://doi.org/10.1007/s11784-014-0185-0

2. Caffarelli, L., Silvestre, L.: An extension problem related to the fractional Laplacian. Commun. Partial Differ. Equ. 32(7-9), 1245-1260 (2007)

3. Caffarelli, L.A., Roquejoffre, J.M., Sire, Y.: Variational problems for free boundaries for the fractional Laplacian. J. Eur. Math. Soc. (JEMS) 12(5), 1151-1179 (2010). https://doi.org/10.4171/JEMS/226

4. Caffarelli, L.A., Salsa, S., Silvestre, L.: Regularity estimates for the solution and the free boundary of the obstacle problem for the fractional Laplacian. Invent. Math. 171(2), 425-461 (2008). https://doi.org/10. 1007/s00222-007-0086-6

5. Cohn, D.L.: Measure Theory, 2nd edn. Birkhäuser Advanced Texts: Basler Lehrbücher. [Birkhäuser Advanced Texts: Basel Textbooks]. Birkhäuser/Springer, New York (2013)

6. Da Lio, F.: Fractional harmonic maps into manifolds in odd dimension $n>1$. Calc. Var. Partial Differ. Equ. 48(3-4), 421-445 (2013)

7. Da Lio, F., Rivière, T.: Sub-criticality of non-local Schrödinger systems with antisymmetric potentials and applications to half-harmonic maps. Adv. Math. 227(3), 1300-1348 (2011)

8. Da Lio, F., Rivière, T.: Three-term commutator estimates and the regularity of $\frac{1}{2}$-harmonic maps into spheres. Anal. PDE 4(1), 149-190 (2011)

9. Da Lio, F., Schikorra, A.: $\frac{n}{p}$-harmonic maps: regularity for the sphere case. Adv. Calc. Var. 7(1), 1-26 (2014)

10. Duzaar, F., Steffen, K.: An optimal estimate for the singular set of a harmonic map in the free boundary. J. Reine Angew. Math. 401, 157-187 (1989)

11. Duzaar, F., Steffen, K.: A partial regularity theorem for harmonic maps at a free boundary. Asymptot. Anal. 2(4), 299-343 (1989)

12. Evans, L.C., Gariepy, R.F.: Measure Theory and Fine Properties of Functions. Studies in Advanced Mathematics. CRC Press, Boca Raton (1992)

13. Fabes, E.B., Kenig, C.E., Serapioni, R.P.: The local regularity of solutions of degenerate elliptic equations. Comm. Partial Differ. Equ. 7(1), 77-116 (1982)

14. Giaquinta, M.: Multiple Integrals in the Calculus of Variations and Nonlinear Elliptic Systems, Annals of Mathematics Studies, vol. 105. Princeton University Press, Princeton (1983) 
15. Gilbarg, D., Trudinger, N.S.: Elliptic Partial Differential Equations of Second Order, 2nd edn. Springer, Berlin (1983)

16. Große-Brauckmann, K.: Interior and boundary monotonicity formulas for stationary harmonic maps. Manuscr. Math. 77(1), 89-95 (1992)

17. Gulliver, R., Jost, J.: Harmonic maps which solve a free-boundary problem. J. Reine Angew. Math. 381, 61-89 (1987)

18. Hardt, R., Lin, F.H.: Partially constrained boundary conditions with energy minimizing mappings. Commun. Pure Appl. Math. 42(3), 309-334 (1989)

19. Heinonen, J., Kilpeläinen, T., Martio, O.: Nonlinear Potential Theory of Degenerate Elliptic Equations, Oxford Mathematical Monographs. The Clarendon Press, Oxford University Press, New York (1993). Oxford Science Publications

20. Hélein, F.: Regularité des applications faiblement harmoniques entre une surface et une sphere. C. R. Acad. Sci. Paris Sér. I Math. 311(9), 519-524 (1990)

21. Hélein, F.: Sur la régularité des applications faiblement harmoniques entre une surface et une variété riemannienne. In: Séminaire sur les Équations aux Dérivées Partielles, 1990-1991, pp. Exp. No. X, 6. École Polytech., Palaiseau (1991)

22. Jost, J.: Riemannian Geometry and Geometric Analysis. Springer, Berlin (2008)

23. Luckhaus, S.: Convergence of minimizers for the $p$-Dirichlet integral. Math. Z. 213(3), 449-456 (1993)

24. Maz'ya, V.: Sobolev spaces with applications to elliptic partial differential equations. In: Grundlehren der Mathematischen Wissenschaften [Fundamental Principles of Mathematical Sciences], vol. 342, augmented edn. Springer, Heidelberg (2011)

25. Millot, V., Sire, Y.: On a fractional Ginzburg-Landau equation and 1/2-harmonic maps into spheres. Arch. Ration. Mech. Anal. 215(1), 125-210 (2014)

26. Millot, V., Sire, Y., Yu, H.: Minimizing fractional harmonic maps on the real line in the supercritical regime. ArXiv e-prints (2017)

27. Morrey Jr., C.B.: The problem of Plateau on a Riemannian manifold. Ann. Math. 2(49), 807-851 (1948)

28. Morrey Jr., C.B.: Multiple Integrals in the Calculus of Variations. Die Grundlehren der mathematischen Wissenschaften, Band 130. Springer, New York (1966)

29. Moser, R.: Partial Regularity for Harmonic Maps and Related Problems. World Scientific, Singapore (2005)

30. Moser, R.: Intrinsic semiharmonic maps. J. Geom. Anal. 21(3), 588-598 (2011)

31. Nash, J.: The imbedding problem for Riemannian manifolds. Ann. Math. 2(63), $20-63$ (1956)

32. Price, P.: A monotonicity formula for Yang-Mills fields. Manuscr. Math. 43(2-3), 131-166 (1983)

33. Rivière, T.: Everywhere discontinuous harmonic maps into spheres. Acta Math. 175(2), 197-226 (1995)

34. Rivière, T.: Conservation laws for conformally invariant variational problems. Invent. Math. 168(1), 1-22 (2007)

35. Schikorra, A.: Regularity of $n / 2$-harmonic maps into spheres. J. Differ. Equ. 252(2), 1862-1911 (2012)

36. Schikorra, A.: $\varepsilon$-regularity for systems involving non-local, antisymmetric operators. Calc. Var. Partial Differ. Equ. 54(4), 3531-3570 (2015)

37. Schoen, R., Uhlenbeck, K.: A regularity theory for harmonic maps. J. Differ. Geom. 17(2), 307-335 (1982)

38. Schoen, R.M.: Analytic aspects of the harmonic map problem. In: Chern, S.S. (ed.) Seminar on Nonlinear Partial Differential Equations (Berkeley, CA, 1983), Math. Sci. Res. Inst. Publ., vol. 2, pp. 321-358. Springer, New York (1984)

39. Simon, L.: Theorems on Regularity and Singularity of Energy Minimizing Maps. Springer, Berlin (1996)

40. Turesson, B.O.: Nonlinear Potential Theory and Weighted Sobolev Spaces, Lecture Notes in Mathematics, vol. 1736. Springer, Berlin (2000) 